\title{
Iconic memory and visible persistence
}

\author{
MAX COLTHEART \\ Birkbeck College, Malet Street, London WCIE 7HX, England
}

\begin{abstract}
There are three senses in which a visual stimulus may be said to persist psychologically for some time after its physical offset. First, neural activity in the visual system evoked by the stimulus may continue after stimulus offset ("neural persistence"). Second, the stimulus may continue to be visible for some time after its offset ("visible persistence"). Finally, information about visual properties of the stimulus may continue to be available to an observer for some time after stimulus offset ("informational persistence"). These three forms of visual persistence are widely assumed to reflect a single underlying process: a decaying visual trace that (1) consists of afteractivity in the visual system, (2) is visible, and (3) is the source of visual information in experiments on decaying visual memory. It is argued here that this assumption is incorrect. Studies of visible persistence are reviewed; seven different techniques that have been used for investigating visible persistence are identified, and it is pointed out that numerous studies using a variety of techniques have demonstrated two fundamental properties of visible persistence: the inverse duration effect (the longer a stimulus lasts, the shorter is its persistence after stimulus offset) and the inverse intensity effect (the more intense the stimulus, the briefer its persistence). Only when stimuli are so intense as to produce afterimages do these two effects fail to occur. Work on neural persistences is briefly reviewed; such persistences exist at the photoreceptor level and at various stages in the visual pathways. It is proposed that visible persistence depends upon both of these types of neural persistence; furthermore, there must be an additional neural locus, since a purely stereoscopic (and hence cortical) form of visible persistence exists. It is argued that informational persistence is defined by the use of the partial report methods introduced by Averbach and Coriell (1961) and Sperling (1960), and the term "iconic memory" is used to describe this form of persistence. Several studies of the effects of stimulus duration and stimulus intensity upon the duration of iconic memory have been carried out. Their results demonstrate that the duration of iconic memory is not inversely related to stimulus duration or stimulus intensity. It follows that informational persistence or iconic memory cannot be identified with visible persistence, since they have fundamentally different properties. One implication of this claim is that one cannot investigate iconic memory by tasks that require the subject to make phenomenological judgments about the duration of a visual display. In other words, the so-called "direct methods" for studying iconic memory do not provide information about iconic memory. Another implication is that iconic memory is not intimately tied to processes going on in the visual system (as visible persistence is); provided a stimulus is adequately legible, its physical parameters have little influence upon its iconic memory. The paper concludes by pointing out that there exists an alternative to the usual view of iconic memory as a precategorical sensory buffer. According to this alternative, iconic memory is postcategorical, occurring subsequent to stimulus identification. Here, stimulus identification is considered to be a rapid automatic process which does not require buffer storage, but which provides no information about episodic properties of a visual stimulus. Information about these physical stimulus properties must, in some way, be temporarily attached to a representation of the stimulus in semantic memory; and it is this temporarily attached physical information which constitutes iconic memory.
\end{abstract}

A predominant theme of modern cognitive psychology, a theme very much in evidence since the renaissance of the subject 20 years ago, is that human beings are encoders and recoders. Much recent experimental work has been devoted to studying the vicissitudes of stimulus information as stimuli are sub-

I am grateful to the following for valuable comments on and criticisms of an earlier draft of this paper: E. H. Adelson, S. Avons, W. P. Banks, C. Blakemore, B. Breitmeyer, V. Di Lollo, R. Fox, J. J. Kulikowski, D. C. Mitchell, P. Mustillo, and N. J. Wade. jected to the various forms of recoding procedures applied, optionally or automatically, to sensory inputs. At the same time, it is recognized that sensory inputs can also exist as mental representations in some relatively unrecoded or unprocessed form. Terms such as "sensory storage" are usually applied to this form of internal representation, and in most information-processing diagrams it is indicated that sensory input exists in an unrecoded form for some short time after the removal of the stimulus: this is considered to be so at least for visual, auditory, and tactile inputs. 
This paper deals only with visual input, though much of what is to be said is applicable to other sensory modalities; and the general aim of the paper is to point out that the view that sensory input exists in the form of an unrecoded internal representation for some time after stimulus offset can be taken to mean three rather different things. It may be that this ambiguity is not important, and that what is involved is merely three different perspectives. On the other hand, the ambiguity may be serious: perhaps these three different concepts of what is meant by the persistence of information in unrecoded form actually have to do with distinctly different processes. We can only clarify the situation by distinguishing between these three approaches to the concept of the persistence of unrecoded visual information and considering exactly how they relate to each other.

What we mean by the term "unrecoded" is that the internal representation of a stimulus shares many of the properties of the stimulus itself. One way in which this might be construed is that the stimulus and its internal representation have in common a reliance on a particular set of neural mechanisms. A second sense is that the stimulus and its internal representation may give rise to indistinguishable phenomenological experiences. The third sense is that an observer may have as much knowledge of specifically visual characteristics of a stimulus when only its unrecoded internal representation is available as when the stimulus itself is still present.

As an illustration of the way in which these three concepts have been treated as interchangeable, consider the term "visual persistence." This term is sometimes taken to refer to the fact that some or all of the neural components of the visual system which respond when a visual stimulus is present continue their activity for some time after the offset of the stimulus. Others use the term to refer to the fact that a visual stimulus continues to be phenomenally present, that is, "visible," for some time after its physical offset; and yet, again, the term is sometimes used to refer to the fact that an observer still possesses a great deal of information about the visual properties of a visual stimulus after stimulus offset.

Correspondingly, one can investigate "visual persistence" in three different ways. One can make neurophysiological recodings of events occurring in the visual system after the offset of a visual stimulus; or one could ask subjects to describe what they see after the offset of a visual stimulus; or one could require them to perform tasks which require that specifically visual properties of the stimulus are accessible after the offset of the stimulus. All three modes of investigation have revealed that there is a visual persistence which exists after stimulus offset and decays away over time. Thus, there is neural visual persistence, phenomenological visual persis- tence, and informational visual persistence. This we know; but do we know what the relationships are between these three senses of the term "visual persistence"? Do we know that neural persistence is visible? Do we know that informational persistence is mediated by neural persistence in the visual system? Do we know that the informational persistence is visible?

As a subject for investigation, the relationships between these three meanings for "visual persistence" have been almost entirely neglected. It has simply been assumed that the question is merely one of a difference in perspective, and that one can treat the three forms of visual persistence as different manifestations of a single entity. For example, the phenomenological and informational persistences are explicitly equated by Julesz:

In the studies of Eriksen et al., the integration of successively presented dots into a form is a special case of the short-term memory integration extensively studied by Sperling. (Julesz \& Chiarucci, 1973, p. 251)

Julesz also treats the neural and informational traces as equivalent:

The "short-term visual memory" of Sperling (1960) is a detailed texture memory, but fades out in $0.1 \mathrm{sec}$. like the afterglow of a cathode ray tube and is merely an afterimage. (Julesz, 1971, p. 103)

The most comprehensive equation of these three senses of the term "visual persistence" is evident in the work of Sakitt $(1975,1976 \mathrm{a}, 1976 \mathrm{~b})$, who takes the view that the informational trace is not only visible but is mediated by the activities of the photoreceptors, especially the rods.

An important consequence of the neglect of these ambiguities was the development of the concept of "direct" and "indirect" methods for studying visual storage (Haber \& Standing, 1969). Indirect methods rely upon subjects retrieving visual information about a stimulus display. Direct methods depend upon phenomenological reports about the visibility of the display. Thus, the former methods concern informational persistence, and the latter phenomenological persistence. Haber and Standing (1970) considered that these two kinds of method provided information about a single entity:

[An] indicator of the visual persistence of a flash... yields data of the same high order of reliability and magnitude found by the far more laborious and indirect procedures of Sperling (1960), Averbach and Coriell (1961) and others.

In order to distinguish as clearly as possible between the three senses of the term "visual persistence," I will use three different terms. One can 
record activity in the visual system for some time after stimulus offset: this I will refer to as "neural persistence." A visual stimulus continues to be experienced for some time after its offset: this I will refer to as "visible persistence"-the term "visible" denoting the fact that the sine qua non of this entity is that it can be seen. Finally, the visual information present in a stimulus continues to be accessible for some time after stimulus offset: this I will refer to as "iconic memory." The topic of this paper is: How are neural persistence, visible persistence, and iconic memory related to each other? Is the relationship one of identity, and, if not, what is it? I hope that it is clear that this is not a philosophical question to do with levels of explanation or reductionism. The three visual traces are straightforward empirically derived concepts, and the question of how they are related to each other is an empirical one.

I will begin with a survey of work on iconic memory, a survey which I consider captures what is the current view of the nature of iconic memory. It may be that this current view is incorrect, and indeed I will hint as much at the end of this paper; but that is not important at present. What is important is to discuss the kinds of experiments which have led to the view that visual information outlasts the stimulus, in a high-capacity rapidly decaying form of visual memory. The methods used in these experiments define the concept "iconic memory," and provide information about the properties of this form of visual persistence. A straightforward way of deciding how iconic memory is related to visible or neural persistence is to decide whether its properties coincide with theirs.

\section{ICONIC MEMORY}

\section{Sperling's Work}

When a display of letters or numbers is presented briefly to an observer-for $500 \mathrm{msec}$ or less-and he is asked to report as many items as he can from the display, it has been known for over a century that observers average about 4 or 5 items correct with displays containing anything from 6 to 18 or more items. The number of items the observer can report is not influenced much by large variations in display duration or in the number of items in the display. Why this limitation on report exists is still unknown. What is known, however, is that the limitation is not a consequence of the observer's inability to store in memory more than 4 or 5 items in the limited time for which the items are on display. This was demonstrated by Sperling (1960); he showed that, with displays as large as 12 or 18 items, all or nearly all of the items are stored in memory, and still exist in this stored form after the offset of the display. The observer's difficulties are introduced by the fact that the form of memory which holds these items, although it is of high capacity, is also subject to rapid decay. This high-capacity rapidly decaying memory mode was subsequently termed "iconic memory" by Neisser (1967).

Sperling demonstrated the existence of iconic memory by an experimental technique known as the partial-report (PR) technique. Suppose displays consist of three rows, each containing four letters, and display duration is $50 \mathrm{msec}$, as in most of Sperling's experiments. If a subject was asked to make a full report (FR), that is, to report as many items as possible from such a display, he averaged about 4.5 items correct, out of a maximum of 12 . However, if he was asked to make a partial report (PR), that is, to report items from only one row of the display, and the required row (top, middle, or bottom) was specified to him after the offset of the display by a tone (high, medium, or low), then he averaged about 3 items correct, out of a maximum of 4. It was completely impossible for the subject to predict before the tone occurred which of the three rows would be requested, because the sequence of tones was completely random. Therefore, if PR performance showed that 3 of the 4 items of the cued row had been stored in memory, it must be that on the average the same number of items from each of the two uncued rows had also been stored in memory. Thus the total number of items which have been stored in memory may be estimated by multiplying the average number of items per row correct in PR performance by the number of rows in the display.

When Sperling did this for a $3 \times 4$ display, the average number of items in memory, as indexed by PR performance was 9.1 . This is more than twice as large as the average number of items correct in FR. The difference, PR - FR, is known as the partialreport superiority.

Having demonstrated that the PR superiority is large with zero cue delay, Sperling then went on to investigate the effect of varying the cue delay. He found that, as cue delay increased, PR superiority decreased until, at cue delays greater than about $300 \mathrm{msec}$, there was no PR superiority. Further increases in cue delay did not impair performance further. The existence of a PR superiority which diminishes as cue delay increases is the principal evidence for the existence of iconic memory.

\section{Two Memory Modes}

Sperling's results show that the form of memory interrogated at short cue delays contains many items, while the form of memory interrogated at long cue delays contains few. Thus, the two forms of memory are quantitatively different. It is usually argued, and will be argued here, that they are qualitatively different also. Before introducing such arguments, it is 
worth considering the simpler view that the difference is merely quantitative. On this view, the relevant memory mode initially holds all or nearly all of the display items, but decays rapidly over a few hundred milliseconds to an asymptotic capacity that is not zero, but about 4.5 items (the number of items to which the declining PR performance asymptotes). No further memory decay occurs, so no further effect of cue delay is apparent. There are many reasons for rejecting this account. The simplest is the observation that the subject's response does not begin until several hundred milliseconds after display offset, and that to respond with four or five letters takes subjects some seconds. If these responses were directly derived from a memory mode which decayed to an asymptotic capacity of 4.5 items in $300 \mathrm{msec}$, the subject would not have access to the preasymptotic high-capacity epoch of this memory. A second reason is that an interfering visual stimulus presented after a display offsetthat is a backward visual mark-reduces performance when presented at delays shorter than the asymptotic cue delay, but not when presented at a much longer delay, 1,000 msec (Lowe, 1975). This illustrates a qualitative difference between the early (high-capacity) and late (low-capacity) memory stages; the former is susceptible to backward visual masking, the latter is not.

For these and many other reasons, one is led to the view, initially proposed by Sperling (1967), that two qualitatively distinct memory modes are involved in the report of brief visual displays. The first is iconic memory: its principal properties are its high capacity and its rapid decay. Its lifetime can be indexed in various ways: by the maximum cue delay at which PR superiority exists, for example, or by the maximum mask delay at which backward masking impairs performance. Under the experimental conditions used by Averbach and Coriell (1961) and Sperling (1960), this lifetime is about $300 \mathrm{msec}$. Since a subject's responses do not begin until some time after the point at which iconic memory has decayed away, these responses must be based upon a second memory mode. This, following Coltheart (1977), will be given the neutral term "durable storage" here, to emphasize the point that, for the purposes of the present discussion, its only relevant property is that its decay time, relative to the time required for the subject to report as many items as he can, is negligible.

In order for items stored in iconic memory to be reported, then, they must first be transferred to durable storage. The FR limitation-only 4.5 items can be given, on the average, in FR-reflects some limitation of this transfer process. It might be that durable storage has a capacity of only 4 or 5 items, though the problem with this proposal is that durable storage is often identified (e.g., by Sperling, 1967) with the acoustically coded short-term memory store (STM) studied in conventional rote-learning experiments, and the results of such experiments indicate that, if the notion of a capacity limitation for STM in terms of number of items is a tenable one, this limitation exceeds considerably a value of 4 or 5 items. An alternative explanation is that the mechanism which transfers items from iconic memory to durable storage has a capacity limitation; after dealing with 4 or 5 items it becomes temporarily incapable of dealing with any more, even if iconic memory still holds untransferred items and durable storage still contains space for them. This explains the FR limitation, but in a rather ad hoc way.

An explanation of the limitation which can be rejected, although it is frequently proposed, is that only 4 or 5 items can be transferred from iconic memory to durable storage because by the time this many items have been transferred the remainder have decayed away. This must be wrong. Increasing exposure duration from 15 to $500 \mathrm{msec}$ must increase the time for which items are available to the transfer mechanism, so should increase the number of items which reach durable storage; yet it has negligible effects on the number of items which can be reported from a display (Sperling, 1960). Furthermore, if the display in a FR experiment is followed by a dark, rather than a light, postexposure field, the maximum cue delay at which a PR superiority occurs is increased (Averbach \& Sperling, 1961), indicating that the useful lifetime of iconic memory has been extended. This should allow more items to be transferred to durable storage, but it does not, because FR performance is not improved when a dark postexposure field is used. Finally, suppose one argued that, in Sperling's experiments, a display duration lasting $50 \mathrm{msec}$ plus an iconic memory lasting up to $300 \mathrm{msec}$ provides enough time for the transfer of 4 or 5 items to durable storage. Assume that the right kind of backward visual mask terminates the useful existence of iconic memory. By varying display duration and presenting this kind of mask at display offset, one would then be investigating directly the relationship between the time available for transferring items to durable storage and the number of items transferred. Such experiments invariably show an initial rapid improvement in number of items reported as display duration increases up to some value between 50 and $100 \mathrm{msec}$, at which value about 4 items can be reported. Further increases in display duration produce much less marked increases in performance: about one item per extra $100 \mathrm{msec}$ at most (for examples, see Coltheart, 1972). Thus items are initially transferred at a high rate, and subsequently at a low rate. This might reflect a property of the transfer mechanism, a property of durable storage, or both; whichever it is, we are compelled to return to 
the first or the second of the explanations of the FR limitation given above, rather than being able to explain this limitation solely in terms of a limitation in the time for which information in iconic memory is available as input to a rather slowly acting transfer mechanism.

Thus, most or all of the items in a display are stored in iconic memory; but only a subset of these items (about 4 or 5 of them) can be transferred into durable storage and hence reported. The existence of PR superiority demonstrates that subjects can choose which of the items in iconic memory are to be transferred. At very short cue delays, where PR performance is perfect, or nearly so, all, or nearly all, of the items of the cued row are transferred to durable storage. As cue delay increases, there will be an increasing probability that a cued item will have decayed from iconic memory before it can be identified as being a cued item and hence can be transferred to durable storage. When the time between display offset and the point at which the cue information is available exceeds the lifetime of iconic memory, selective transfer into durable storage of cued, rather than uncued, items will no longer be possible.

\section{Selective and Nonselective Transfer}

This raises the question as to what a subject will be doing in the interval between display offset and cue availability. If the answer is "nothing"-if no information is input to durable storage until the subject knows which is the cued row-then, on occasions when cue information becomes available only after iconic memory has decayed completely, no items will reach durable storage at all, and so no items can be reported. Although an effect of this kind has been reported (von Wright, 1968), the usual finding is that PR performance at cue delays exceeding the lifetime of iconic memory is equal to FR performance. This suggests that a common strategy for the subject is to begin transfer into durable storage as soon as he can, even though he does not yet know which are to be the cued items. This will guarantee that PR performance cannot be any worse than FR performance. Such "nonselective readout" (Averbach \& Coriell, 1961), here termed nonselective transfer, continues until information as to which are the cued items is available. Then the transfer process switches to these items only: "selective readout" or selective transfer. The interplay between these two processes (note, for example, that a cued item can still reach durable storage via the nonselective readout process) has been most elegantly analyzed by Averbach and Coriell (1961) (their analysis is discussed by Coltheart, 1977), but many unsolved problems remain. Once again, it is the mysterious FR limitation which causes the difficulties. Since about 4 items can be transferred during the lifetime of a display of $50 \mathrm{msec}$, and since once this many items have been transferred transfer becomes very slow (either because the process itself no longer works well or because durable storage is nearly full), a subject who employs nonselective transfer before the cue information is available should have great difficulty in selectively transferring items, even if he begins doing so at or very soon after display offset. Yet, this does not happen: all or nearly all the cued items can be transferred to durable storage if the cue follows soon enough after display offset.

Sperling (1960; see also Sakitt, 1976a, p. 260) noted that strategic effects were important here. At long cue delays, subjects employed nonselective transfer; at short delays, they avoided this, waiting for the cue before doing any transfer. Figure 4 of Sakitt (1976a) shows that her subject employed a particular nonselective transfer strategy (transferring top-row items; this is nonselective with respect to the cue) at cue delays of $1 \mathrm{sec}$ or more, but not at very short delays. Clearly, a subject's ability to use one or the other form of transfer as a function of what the cue delay is will be much greater if cue delay is blocked (therefore predictable in advance) than if it is randomized (therefore unpredictable). Sperling (1960, pp. 8-10) dealt with this issue and showed, for example, that one of his subjects (R.N.S.), in an experiment where cue delay was blocked and therefore predictable, relied on waiting for the cue and selective transfer at cue delays up to and including $150 \mathrm{msec}$, but used nonselective transfer as well as selective transfer at cue delays of 300 and $500 \mathrm{msec}$. If the use of nonselective transfer cost the subjects nothing, such patterns of results would not occur; the subject could simply use nonselective transfer until the cue had been decoded. Since, at short cue delays, where selective transfer is likely to be possible, subjects eschew nonselective transfer, this implies that they consider that some benefit accrues from not carrying out nonselective transfer. This benefit might be avoiding overcrowding of durable storage, or it might be avoiding overtaxing of the transfer mechanism. Further work on this issue thus might provide some insight into the nature of the FR limitation.

\section{Forms of Information in Iconic Memory}

Since one can selectively transfer items from iconic memory according to the spatial locations they had occupied in the display, information about an item's spatial location must be represented in iconic memory. Following this reasoning, one can discover which forms of information exist in iconic memory by determining which forms of information can serve as the basis for selective transfer. In practice, this means finding which forms of postdisplay cueing produce a PR superiority. Strictly speaking, various 
cue delays ought always to be used, so as to demonstrate that the PR superiority declines as cue delay increases. If this is not done, it is less easy to dismiss alternative explanations of PR superiority, such as "output interference." If reporting items from durable storage cause some of its contents to be lost before they can be reported, an apparent PR superiority might ensue simply because fewer items need be output from durable storage under PR conditions than under FR conditions-for example, if there are three rows in a display, to achieve equal PR and FR performance one must output from durable storage three times as many correct items in the FR condition than in the PR condition. It is very unlikely that output interference effects are large enough to contribute much to PR superiorities (for arguments about this, see Coltheart, 1975a, 1975b; Coltheart, Lea, \& Thompson, 1974; Holding, 1970, 1972, 1973, 1975b); but even if they were, the PR superiority would not vary as a function of cue delay, and thus it is desirable, even though not essential, to include cue delay as a variable when studying PR superiorities. This has sometimes, but not always, been done.

It has been found that a variety of cueing criteria can produce PR superiorities: color (Clark, 1969; Coltheart, Lea, \& Thompson, 1974; Dick, 1969; von Wright, 1968), brightness (von Wright, 1968), shape (Turvey \& Kravetz, 1970), direction of rotary or linear motion (Demkiw \& Michaels, 1976; Russell, 1977; Treisman, Russell, \& Green, 1975), and flicker (Russell, 1977). Thus, a large variety of visual properties of items are represented in iconic memory. It should be noted here that, as Sakitt (1976a, p. 270) points out, the varying colors used in experiments with color as the partial-report criterion were not matched for brightness. Therefore, they may have differed in brightness too; and since partial report based upon brightness as a cue is possible (von Wright, 1968) one cannot be sure from the color experiments that color can be used as a criterion for selective transfer from iconic memory. Adelson (1978) and Banks and Barber (1977) used displays in which letters and background were scotopically matched, that is, the letters could be discriminated only by the cone system. With display row as the partial-report criterion, a conventional decaying partial report superiority was obtained. This suggests that, even with scotopic matching, color itself should be usable as a selection criterion. ${ }^{1}$

Two stimulus properties which cannot be utilized by subjects for selective transfer from iconic memory are (1) whether an item is a letter or a digit (Sperling, 1960; von Wright, 1968); and (2) whether a letter's name does or does not contain the phoneme "ee" (Coltheart, Lea, \& Thompson, 1974). The absence of a PR superiority in each of these conditions suggests that iconic memory does not contain semantic or phonological information, and this conclusion, plus the evidence that PR superiority occurs with a variety of visual criteria, is taken as evidence that iconic memory is a precategorical store containing solely visual information.

The experiments on iconic memory just described, and others like them, illustrate the way in which the essence of the concept is that it refers to a decaying store of visual information. These experiments do not require that this information be visible (though this may be so), or that its neural basis be persisting activity in the visual system (though this may be so too). The iconic memory experiments require subjects to process visual information after the visual stimulus has been terminated; by contrast, experiments on visible persistence depend upon subjects' experiences of visual sensation after stimulus offset. I turn now to a discussion of these phenomenological experiments.

\section{VISIBLE PERSISTENCE}

Numerous methods have been developed over the past decade for studying visible persistence, and it is convenient to classify experiments according to the particular experimental method used.

\section{Judgment of Synchrony}

If a visual test stimulus remains visible for some time after its physical offset, one can measure the duration of its visible persistence by adjusting the temporal location of a probe stimulus until the onset of the probe stimulus and the offset of the test stimulus seem to be simultaneous. This technique was introduced by Sperling (1967), and has been more extensively explored by Bowen, Pola, and Matin (1974), Efron (1970a, 1970b, 1970c), and Haber and Standing (1970).

Efron (1970a) used, as his test stimulus, an orange disk of light, $1 \mathrm{deg} 38 \mathrm{~min}$ in diameter, located $3 \mathrm{deg}$ $8 \mathrm{~min}$ to the left of a fixation point, with a luminance of $2.6 \mathrm{fL}$, at durations varying from 10 to $500 \mathrm{msec}$. It was viewed binocularly, and preceded and followed by darkness. The subject's task was to synchronize the offset of this stimulus with the onset of the probe stimulus, a green disk of light, $1 \mathrm{deg} 38 \mathrm{~min}$ in diameter, located $3 \mathrm{deg} 8 \mathrm{~min}$ to the right of the fixation point; the luminance of the probe stimulus was $1.2 \mathrm{fL}$ and its duration $500 \mathrm{msec}$. That interval between the physical offset of the test stimulus and the physical onset of the probe stimulus for which these two events are judged to be simultaneous is a measure of the duration for which the test stimulus persists visibly after its physical offset. The relationship between the duration of visible persistence and test-stimulus duration found by Efron is shown in Figure 1a. A very similar result was 
obtained when the test stimulus was a burst of white noise and the probe stimulus a $2,000-\mathrm{Hz} 500-\mathrm{msec}$ tone, that is, when the duration of audible persistence was being measured.

Figure 1 suggests that a visual stimulus of duration $130 \mathrm{msec}$ or more persists visibly for a fixed amount of time (here about $100 \mathrm{msec}$ ) after its offset, regardless of what its duration is, whereas a visual stimulus of duration less than 130 msec persists for an amount of time which is inversely related to stimulus duration, this relationship taking the form: stimulus duration + duration of persistence $=$ constant (here about $230 \mathrm{msec}$ ). An alternative description of these results is that any visual stimulus appears to last for a minimum period of $230 \mathrm{msec}$; stimuli whose physical durations are in the range 0 to $130 \mathrm{msec}$ all appear to last for $230 \mathrm{msec}$, whereas stimuli whose durations exceed $130 \mathrm{msec}$ appear to last for a period
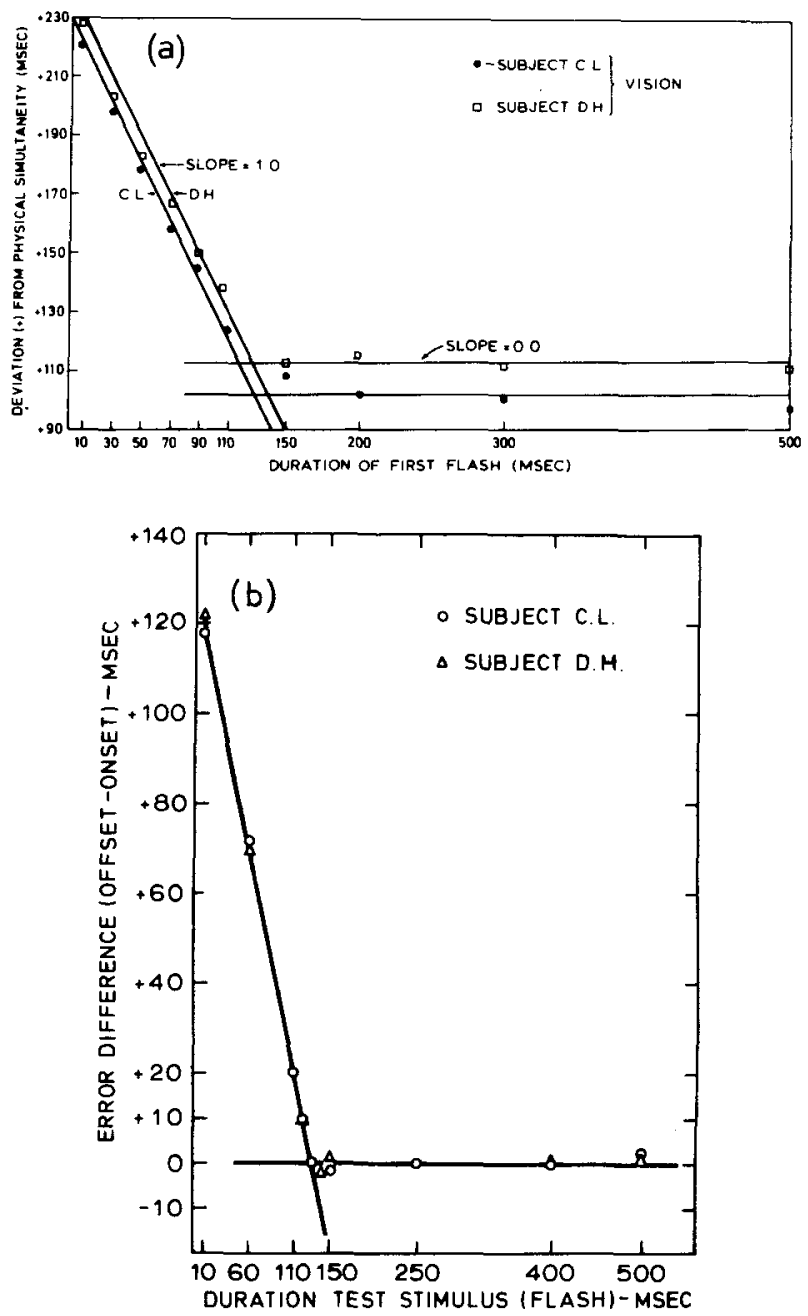

Figure 1. (a) Visual persistence measured with a visual probe stimulus (Efron, 1970a). (b) Visual persistence measured with an acoustic probe stimulus (Efron, 1970c). equal to their actual duration plus $100 \mathrm{msec}$. This form of description of his results was used by Efron (1970a): "Perceptions have a minimum duration, produced by stimuli shorter than the critical duration, and ... perceptions evoked by stimuli longer than this critical value are continuously graded with respect to duration."

A similar experiment is described in Efron (1970c). Here the test stimulus was a 1-deg 40-min red disk of light, of luminance $3.4 \mathrm{fL}$, viewed monocularly through an artificial pupil, presented foveally, and preceded and followed by darkness. The probe stimulus was a very brief auditory click. The relationship between the duration of visible persistence of the test stimulus and the duration of the test stimulus is shown in Figure $1 \mathrm{~b}$. The two sets of results given in this figure are very similar. The functions consist of two linear segments, an initial one with a slope of -1.0 followed by a second segment with a slope of 0.0 . However, there is an obvious and major difference between the two sets of results: Although, in both sets, stimuli longer than $130 \mathrm{msec}$ persist for an amount of time that is independent of stimulus duration, this amount is about $100 \mathrm{msec}$ in the results of Efron (1970a) and zero in the results of Efron (1970c). Thus, the latter results indicate that visual stimuli whose durations exceed $130 \mathrm{msec}$ have no visible persistence at all, while the former indicate that such stimuli yield visible persistences lasting about $100 \mathrm{msec}$. How might this conflict between the two sets of results, a conflict which neither Efron nor others have noted, be explained?

One possibility is that the difference in results might be due to differences between rods and cones. In the experiment showing zero persistence for longer stimuli, the test stimulus was red and foveal, and so its perception would rely largely on cone vision; in the other experiment, since the test stimulus was orange and parafoveal, both its wavelength and retinal location would permit somewhat greater rod involvement. Perhaps longer stimuli generate visible persistence in rods only, not in cones. This would be consistent with the conclusions of Bartlett, Sticht, and Pease (1968), whose work is discussed below; using a different experimental technique, they concluded that stimuli with durations in the range 2 to $4 \mathrm{sec}$ generated visible persistence when presented peripherally (15 deg away from the fovea) but not when presented foveally.

This explanation for the differences between Efron's two sets of results can be rejected, however, because precisely the same kind of difference occurred when the persistence of auditory stimuli was measured. That is, auditory stimuli lasting longer than $130 \mathrm{msec}$ yielded nonzero persistences (of about $50 \mathrm{msec}$ ) when the probe stimulus was a tone, but zero persistences when the probe stimulus as a 5 -msec flash. Thus, 
for stimuli longer than $130 \mathrm{msec}$, zero persistence occurs when test stimulus and probe stimulus are in different sense modalities; nonzero persistence occurs when test and probe stimuli are in the same modality. This pattern of results cannot be explaind in terms of differential latency of response to the onset of visual compared to auditory stimuli, since such an explanation is ruled out by the symmetry of the effects. If, for example, visual stimuli had longer onset latencies, this might explain why persistence of an auditory test stimulus was not evident when the probe stimulus was visual, since the longer latency for the visual stimulus would require it to be set earlier in time; but, if this were so, the use of an auditory, rather than a visual, probe stimulus when measuring persistence of a visual stimulus would produce increased, not decreased, values for the duration of visible persistence. In any case, Efron (1970c) showed that when subjects were required to synchronize the onsets of his visual and auditory stimuli, their settings resulted almost exactly in physical simultaneity; thus, the two types of stimulus did not differ in onset latencies in his experiment.

Why should long stimuli show persistence when the probe stimulus is in the same modality, but not when it is in a different modality? I will consider this point below, but before doing so will discuss other results of experiments, using synchrony judgments to measure the duration of visible persistence.

In Efron's cross-model experiment, the effect on persistence duration of varying test-stimulus intensity was explored. Persistence duration for a $60-\mathrm{msec}$ visual stimulus decreased as intensity increased; an increase of $2 \mathrm{log}$ units in intensity reduced persistence duration from about 150 to $67.6 \mathrm{msec}$.

Haber and Standing (1970) used crossmodal synchrony judgments: Their test stimulus was a $3 \times 3$ array of black letters or squares on a 5-deg-diam light background whose luminance was $5 \mathrm{~mL}$ (4.65 fL). The array subtended a visual angle of $1 \mathrm{deg} 6 \mathrm{~min}$ and was fixated at its center. The probe stimulus was a 1-msec click, which was adjusted by the subject until it appeared simultaneous with the onset of the test stimulus (in one block of trials) or with the offset of the test stimulus (in another block of trials). The interval between these two settings represents the apparent duration of the stimulus (the time for which it was physically present plus the time for which it persisted visibly). Persistence duration was estimated by subtracting stimulus duration from the interclick interval. The functions relating persistence duration to stimulus duration for various combinations of pre- and postadapting conditions are shown in Figure 2.

These functions are much more variable than those obtained by Efron and shown in Figure 1. However, there is reasonable agreement (at least when Haber

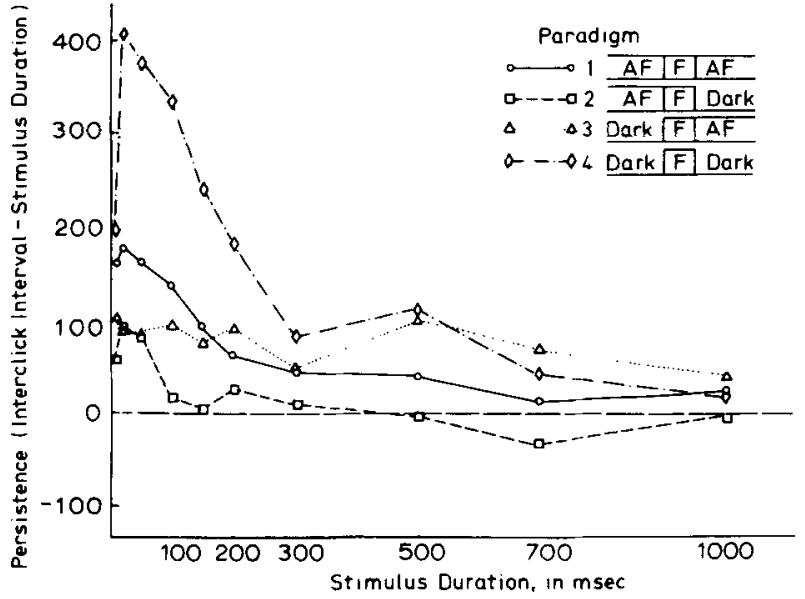

Figure 2. The results of Haber and Standing (1970).

and Standing's pre- and postexposure fields have the same luminance). For brief stimuli, persistence duration decreases sharply as stimulus duration increases. For longer stimuli, persistence duration is unaffected by varying stimulus duration. Unfortunately, it is difficult to decide from this figure whether there is significant persistence at long stimulus durations, and Haber and Standing did not carry out any statistical analysis relevant to this question. Thus these data do not throw much light upon the suggestion that when test and probe stimuli are in different modalities, longer stimuli will have zero persistence, a suggestion made earlier in connection with Efron's work, although the result obtained at a stimulus duration of $500 \mathrm{msec}$ with dark pre- and postfield, a persistence of $100 \mathrm{msec}$, does not fit this suggestion well.

When the prefield was dark and the postfield light, the pattern of results obtained was quite unlike that yielded by the other three conditions; and when the prefield was light and the postfield dark, persistences were shorter than with both prefield and postfield light, whereas in the dark/dark condition persistences were longer than in the light/light conditon. As Haber and Standing (1970, p. 226) acknowledge, "the effects of adaptation on persistence are not ordered in any particularly clear fashion .... the pattern of results is puzzling, but further delineation of the four conditions is needed before further speculation would seem to be warranted." When a light preor postfield was used, it had the same luminance as the display, so onset or offset settings, respectively, could not be made with respect to the display as a whole, only with respect to the letters it contained. Whether and when the subject was using the display as a whole or just the letters cannot be determined. They also note that "under some paradigms the letters of the stimulus were invisible at the shortest duration used. In these cases, a black square was 
substituted for the letters." This may have happened at the shortest exposure in the dark/dark condition, which yielded an abnormally short duration of visible persistence.

When the display was followed, at varying ISIs, by a patterned mask, offset clicks were set at mask onset when the ISI was less than estimated persistence duration and before mask onset when the ISI was greater than estimated persistence duration, suggesting that the mask onset terminates the visibility of the display's persistence unless this persistence has already ended before the mask is presented. An unpatterned (homogeneous light) backward masking stimulus did not terminate persistence, but instead reduced its duration.

A final point which might be made about these results is that the slope of the function relating persistence duration to stimulus duration for short durations, which was about -1.0 in Efron's experiments, is about -1.0 in the dark/dark condition of Haber and Standing's (neglecting the shortest duration), but appears to be somewhat shallower in the light/ light condition (see Figure 2).

Bowen, Pola, and Matin (1974) used intramodal synchrony judgments to study visible persistence. Their test stimulus was a 10-min-diam disk of light, located $30 \mathrm{~min}$ to the right of a fixation point, presented at varying luminances and durations. Their probe stimulus was a 3.8-min-diam disk of light, located $2 \mathrm{deg} 30 \mathrm{~min}$ to the left of a fixation point, and presented for $1 \mathrm{msec}$ at $6.31 \mathrm{fL}$. Viewing was monocular and subjects were dark adapted.

In their first experiment, two test-flash luminances were used ( 2.82 and $.32 \mathrm{fL})$, and test-flash duration was varied from 5 to $350 \mathrm{msec}$. Two subjects were used. The brighter test flash yielded shorter persistence values, an increase of $.94 \mathrm{log}$ units producing persistence decrements of 48 and $21 \mathrm{msec}$ for the two subjects, results consistent with those of Efron (1970c). One subject (J.P.) yielded results like those obtained by Efron and by Haber and Standing. The function relating persistence duration to stimulus duration was reasonably well fitted by two straight lines, an initial segment with a slope of -1.00 and a subsequent segment with a slope of zero. The other subject produced a function whose slope was much shallower (about - .5). However, in a second experiment, using similar test flash luminances with testflash durations of $25,100,250$, and $1,000 \mathrm{msec}$, both subjects showed slopes of about -1.00 over the duration range 25 through $250 \mathrm{msec}$, for both test flash luminances; and the results with the 1,000 -msec test flash were consistent with the existence of a second segment of this function having a zero slope and beginining at a stimulus duration between 250 and $300 \mathrm{msec}$. It whould be noted that the use of a 4-mm-diam artificial pupil in this experiment would have considerably reduced the retinal illumination produced by the test flashes, so that the finding of steeper slopes here than in Experiment 1 is not entirely consistent with the evidence (see below) that reducing stimulus intensity makes the slope of the function relating persistence duration to stimulus duration more shallow.

This issue was further pursued by investigating, with subject A.C., how the slope of the persistence/duration function was influenced by stimulus intensity. As luminance was reduced, this slope became shallower and shallower. At the maximum luminance used $(2.82 \mathrm{fL})$, the slope was about -.50 (these were A.C.'s data from Experiment 1), but, since A.C. yielded a slope near -1.0 at this luminance in the second experiment, it seems reasonable to suppose that with dark-adapted subjects a luminance of about 2-3 $\mathrm{fL}$ is needed to produce a slope of -1.0 . Efron used luminances of 2.6 and $3.4 \mathrm{fL}$, and Haber and Standing, a luminance of $4.65 \mathrm{fL}$; all obtained slopes of about -1.0 with dark-adapted subjects. With a light-adapted subject, Haber and Standing obtained a slope somewhat shallower than -1.0 . This slope is an important parameter, and if it is equal to -1.0 , indicating the existence of some "minimum duration of a perception," to use Efron's term; that is especially significant. Bowen et al.'s results might indicate that the generation of such a minimally short perception requires a certain critical stimulus intensity; at subcritical intensities, durations longer than the minimum duration are generated.

Since increasing flash duration has the same effect on persistence duration as increasing flash luminance, Bowen et al. next investigated the possibility that the relevant variable was flash energy. They used a variety of duration/luminance combinations, all having the same energy: $.014 \mathrm{fL} / \mathrm{sec}$. Duration varied from 5 to $374 \mathrm{msec}$ and, correspondingly, luminance varied from 2.8 to $.038 \mathrm{fL}$. For durations from 5 to $100 \mathrm{msec}$ (or, equivalently, for luminances from 2.8 to $.14 \mathrm{fL}$ ), duration of persistence was constant for these equal-energy stimuli. Beyond this point, persistence duration declines as duration increased and luminance decreased. The fact that persistence duration declined indicates that beyond this point stimulus duration was of more importance than luminance in determining persistence duration. This is not because the effect of luminance on persistence time is lessened at increasing durations, since this effect is constant at durations in the range of 25 to $1,000 \mathrm{msec}$ (Bowen et al., 1974, Figure 4).

It was pointed out earlier that, while it is reasonably clear that the relationship between stimulus duration and the duration of visible persistence has a zero slope for stimulus durations beyond some critical point (a result also found by Bowen et al.- - see their Figure 7b), what is not clear is whether stimuli 
whose duration exceeds this critical value generate any visible persistence at all. Zero persistence was observed in the cross-modal experiment of Efron (1970c), while the cross-modal experiment of Haber and Standing (1970) yielded unclear results. When the persistence generated by a vibrotactile test stimulus was measured cross-modally, using either a visual or an auditory probe stimulus, test stimuli longer than about 130 msec yielded zero persistence (Efron, 1973). On the other hand, the intramodal experiments of Bowen, Pola, and Matin (1974) and Efron (1970a) yielded persistences of between 100 and $200 \mathrm{msec}$ for stimulus durations exceeding the critical duration.

If it took over $100 \mathrm{msec}$ to switch attention from one location in visual space to another (or one auditory stimulus to another), but only a negligible amount of time to switch attention from a visual stimulus to an auditory stimulus (or vice versa), this pattern of results would emerge. However, this proposal is not particularly plausible, and, in additon, if the 100-msec "persistences" obtained with relatively long-lasting test stimuli and within-modality probe stimuli are attention-switching artifacts, why are their durations inversely related to stimulus intensity, as shown by Bowen et al. (1974)? The question of whether or not relatively long-lasting stimuli generate any visible persistence thus remains unsolved.

Sakitt (1976a, 1976b) has also used a variant of the synchrony-judgment technique. Her stimulus was a $2 \times 6$ matrix of white letters presented for $50 \mathrm{msec}$ on a black ground, with black pre- and postexposure fields. A 20 -msec tone was presented at some time after the onset of the display. The subject's task was to vary the delay of the tone until it appeared simultaneous with the disappearance of the display. When duration of visible persistence measured in this way was plotted against stimulus intensity measured in log units above rod threshold, persistence duration was constant over the range 0 to $5.5 \mathrm{log}$ units of intensity; beyond that intensity, increasing intensity produced increases in the durations of persistence This result conflicts grossly with the other work using this technique, discussed earlier. Why?

The answer appears to have to do with the stimulus luminances used. According to Sakitt (1976b, p. 136), the stimulus luminance beyond which persistence duration begins to increase is about $3.16 \mathrm{log}$ scotopic trolands. This corresponds to a luminance considerably greater than any used in the other experiments on visible persistence I have described. Consequently, it appears that with relatively high luminances, one can obtain a positive relationship between persistence duration and stimulus intensity (this is consistent with Sakitt's threefold classification of afterimages, discussed later).

This does not explain why, with lower luminances, Sakitt $(1976 \mathrm{a}, 1976 \mathrm{~b})$ found that persistence duration was independent of luminance, whereas the other studies (and many more to be discussed) found that there was an inverse relationship in this luminance range. However, Sakitt (1976a, p. 266) notes that her method of measuring persistence duration was an insensitive one for durations measuring some hundreds of milliseconds; and from statements by Sakitt (1976b), one can calculate that a true persistence of, for example, $125 \mathrm{msec}$ might come out as low as $79 \mathrm{msec}$ or as high as $198 \mathrm{msec}$ in her experiments. It is safe to conclude, then, that with low luminances and persistences of about 200 or $300 \mathrm{msec}$ there is, in fact, an inverse relationship between persistence duration and stimulus luminance, even though this was not evident in Sakitt's data. At high luminances, on the other hand, there is a direct relationship between persistence duration and stimulus luminance. This is unlikely to be relevant to most of the work discussed in this paper, because conventional tachistoscopes operated well below the luminance at which a direct luminance/duration relationship emerges.

The synchrony-judgment technique has also been used by Adelson (1978); his work is discussed below, in the section dealing with rods and cones.

To summarize: The studies using the synchronyjudgment technique have shown that brief stimuli (those lasting up to 150 to $200 \mathrm{msec}$ ) generate a visible persistence whose duration is inversely related to both stimulus duration and stimulus luminance (provided that stimulus luminance does not considerably exceed the maximum luminance characteristic of most tachistoscopic experiments). The slope of the function relating duration of visible persistence to stimulus duration has a slope of -1.0 , except for stimuli with very low luminance; for these stimuli, the slope becomes shallower as luminance decreases. Persistence durations are considerably longer when pre- and postfields are dark than when they are light.

What happens with longer stimuli (in excess of $200 \mathrm{msec}$ ) is unclear. Experiments in which test and probe stimuli are in the same modality suggest that these longer stimuli have a persistence of $100-200 \mathrm{msec}$, regardless of stimulus duration. Experiments in which probe and test stimulus are in different modalities suggest that long stimuli do not generate any visible persistence at all. This problem requires investigation.

\section{The Onset/Offset Reaction Time Technique}

Instead of asking a subject to adjust a probe stimulus so that it is simultaneous with the apparent onset or offset of a test flash, one could simply ask the subject to signal the apparent onset and of fset of the flash by making some kind of response, for example, pressing a button. If a display persists visibly for some time after its physical offset, this may prevent the subject from detecting the true physical offset; instead, he may produce an offset response only when the visible persistence of the display has been 
terminated. In this case, the time between the physical offset of the display and the subject's offset response will include a component representing the persistence time of the display. Briggs and Kinsbourne (1972) proposed that this component could be isolated if one also measured reaction time to the onset of the display. They assumed that onset and offset RTs would consist of identical components, except for the component of offset RT due to visible persistence. If so, subtracting onset RT from offset RT would yield a measure of the duration of visible persistence.

It is fairly obvious that this technique cannot be taken seriously as an indicator of the absolute duration of visible persistence. If such persistence decays exponentially, the decision as to when it has terminated will be an extremely difficult one. It could only be made by adopting some criterion of the form "Respond when persistence strength has fallen below level $k$," and the choice of the value for $k$ could only be arbitrary. Since stimulus onsets will have steep rise times, this problem will not occur for onset decisions. Thus, the actual values one obtained for the durations of visible persistences using this method cannot be precise, since they will depend, to some extent, upon the criterion adopted by the subject. ${ }^{2}$

However, the technique may still be useful for measuring the relative durations of visible persistences in a variety of situations. If one can assume that a subject adopts a consistent criterion across a variety of experimental situations, differences in persistence times as a function of situation can be taken seriously. Thus, it is worth considering the results obtained using the offset-minus-onset-RT technique.

Although Briggs and Kinsbourne (1972) were the first to treat this technique as providing an index of visible persistence, a number of earlier studies had investigated the differences between RT to onset and offset of visual stimuli. The earliest studies, carried out in the early part of this century, were methodologically inadequate; therefore, only recent work will be discussed here.

Rains (1961) used a centrally fixated high-intensity (344 mL) white stimulus subtending $12 \mathrm{deg} 44 \mathrm{~min}$ of visual angle. His subjects were not dark adapted. Stimulus duration was not specified in his paper, but offset RTs were measured with long-duration stimuli, and it appears that onset RTs were measured with very brief stimuli. Rains found no differences between onset, RTs and offset RTs. A more extensive study was carried out by Pease and Sticht (1965). They used a small target $(20 \mathrm{~min}$ in diameter) and presented it either to the fovea or to a point $15 \mathrm{deg}$ from the fovea. In the offset-RT condition, stimulus durations in the range 2-4 sec were used, the durations being randomized within this range. Four luminances were used. The subjects were dark adapted.
For the fovea, they obtained the same results as Rains (1961), namely, no differences between onset RTs and offset RTs. They suggested that offset RT was shorter than onset RT in the fovea; but, in an analysis of variance, this effect yielded an $F(1,2)=3.03$, $\mathrm{p}>.20$. However, in the periphery, offset RTs were slower than onset RTs by up to about $70 \mathrm{msec}$. This could be taken to imply that the peripheral stimuli generated visible persistence, while the foveal stimuli did not. The duration of persistence decreased as stimulus intensity increased; the most intense stimulus $(3,140 \mathrm{~mL})^{3}$ generated no persistence.

This work was followed up by Bartlett, Sticht, and Pease (1968), whose experiment was very similar to that of Pease and Sticht except that the stimulus was colored, either red (wavelengths above $650 \mathrm{~nm}$ ) or blue (wavelengths below $495 \mathrm{~nm}$ ). They confirmed the earlier findings: no persistence in the fovea, persistences of up to about $80 \mathrm{msec}$ in the periphery. For the blue stimulus, persistence duration decreased as stimulus intensity increased (as Pease and Sticht had found for a white stimulus). For the red stimulus, this relationship did not hold; durations increased, then decreased, as luminance was varied from .0129 to $1.29 \mathrm{~mL}$.

Comparisons of the RT results with the two different hues, in relation to the functions relating hue to sensitivity for rods and for cones, allowed Bartlett et al. to take the view that the onset and of fset RTs to the foveal stimuli were generated via the cone system, whereas onset and offset RTs to the peripheral stimuli were generated via the rod system. This implies that, under the conditions of their experiment, cones do not generate visible persistence, whereas rods do. Adelson (1978) has concluded that rods generate longer visible persistences than cones; his work is discussed later.

Here it is important to note that Rains, Pease, and Sticht and Bartlett et al., all of whom found no persistence for foveal stimuli (Rains' stimulus was large, but was centrally fixated), all used stimuli lasting for at least $2 \mathrm{sec}$ when measuring offset RTs. If the duration of visible persistence is inversely related to stimulus duration, as the results of experiments using judgments of synchrony show, then it remains possible that briefer stimuli would reveal some foveal visible persistence with the onset/offset RT technique. It may be, for example, that the maximum duration at which persistence still occurs is shorter for the fovea than for the periphery.

It was noted earlier that the synchrony-judgment technique yielded ambiguous results concerning the question of whether relatively long-lasting stimuli have any visible persistence at all. Intermodal experiments suggested that they did not; intramodal experiments suggested that they did. The stimuli in these synchrony experiments were all foveal or parafoveal. 
The results from onset/offset RT experiments, which suggest that relatively long-lasting stimuli do not generate foveal visible persistence, thus agree with the intermodal synchrony experiments, and not the intramodal synchrony experiments; but if this is a genuine agreement, it would have to be that even an intermodal synchrony experiment would demonstrate visible persistence for longer-lasting stimuli if these stimuli were presented peripherally.

There has been only one study of visible persistence which used both the onset/offset RT technique and stimuli of brief duration, that of Briggs and Kinsbourne (1972). The displays they used were $3 \times 3$ matrices of letters or squares, black on a white field of luminance $4 \mathrm{fL}$. The displays subtended $6 \mathrm{deg} 26 \mathrm{~min} \times 6 \mathrm{deg} 26 \mathrm{~min}$ and were centrally fixated. When the display was absent, an adapting field of luminance $4 \mathrm{fL}$ was present. Persistence of a monoptic letter display declined from 74 to $11 \mathrm{msec}$ as stimulus duration increased from 100 to $1,000 \mathrm{msec}$. Persistence of a monoptic squares display declined from 41 to $13 \mathrm{msec}$ in the same duration range, and this display yielded persistences of less than $10 \mathrm{msec}$ for stimulus durations between 1,500 and $3,000 \mathrm{msec}$. For the reason given earlier, one cannot attach much significance to these absolute values; but one can claim that Briggs and Kinsbourne have shown an inverse relationship between persistence duration and stimulus duration.

In addition to these two monoptic conditions, Briggs and Kinsbourne ran a third condition using dichoptic letter displays; half-way through the display duration, the display was switched from the nondominant eye to the dominant eye. The reason for using this condition was as follows. A $100-\mathrm{msec}$ monoptic display yielded a persistence of about $74 \mathrm{msec}$. If it were followed, with zero ISI, by an identical display to the other eye and this second display lasted for $100 \mathrm{msec}$, it should generate its own 74-msec persistence. Thus, if persistence were purely a monocular process, a 200 -msec dichoptic stimulus should yield the same persistence as a 100 -msec monoptic stimulus $-74 \mathrm{msec}$, in this example. This did not happen; persistence was a function of total stimulus duration and was not influenced by whether the stimulus was presented monoptically or was switched from one eye to the other half-way through its lifetime. Briggs and Kinsbourne (1972) therefore concluded as follows:

If persistence were a peripheral (retinal) phenomenon, the persistence of a $200 \mathrm{msec}$. dichoptic stimulus would be equal to the persistence of a $100 \mathrm{msec}$. monoptic stimulus. It is in fact comparable to the persistence of a $200 \mathrm{msec}$. monoptic stimulus. This indicates that the persistence mechanism is central, operating after the confluence of the inputs from the two eyes.

At least two objections could be raised to this conclusion. The first is that, in the dichoptic condition, the stimulus was switched from one eye to the other when $50 \%$ of the total stimulus duration had elapsed. If the subject could detect this switching in any way, it provided him with a means of anticipating when the stimulus was to terminate, since the switching occurred exactly halfway between stimulus onset and stimulus offset. ${ }^{4}$ This may be why offset reaction times were faster in the dichoptic condition, rather than the cause being the central nature of visible persistence. A second objection is that the subject may detect independently the two stimulus offsets, one per eye, but be unsure which is the one to which he should respond. This would produce a proportion of early responses (responses to offset of the leading stimulus) which would make the mean offset RT artefactually short. These objections could be met if the dichoptic task were carried out with random variation of the proportion of total time devoted to the leading stimulus and if it could be shown that RT. variance was no greater in the dichoptic than in the monoptic condition. In the absence of these precautions, we cannot be sure that the visible persistence studied by Briggs and Kinsbourne is a central process.

Briggs and Kinsbourne's data agree with those reported by Rains (1961); both experiments used fairly large displays (6 deg $26 \mathrm{~min}$ square and $12 \mathrm{deg} 44 \mathrm{~min}$ square, respectively) with central fixation of the display, and both found that displays lasting for a second or more yielded negligible persistence. Bartlett, Sticht, and Pease (1968) and Pease and Sticht (1965) found the same result with small foveal stimuli lasting for between 2 and $4 \mathrm{sec}$. However, persistences of up to about $80 \mathrm{msec}$ are found with the onset RT/offset RT method if either: (a) the stimuli are peripheral (Bartlett, Sticht, \& Pease, 1968; Pease \& Sticht, 1965), even with long-duration stimuli; or (b) the stimuli are brief (Briggs \& Kinsbourne, 1972), even with foveal stimuli.

One might argue that the RT technique is intrinsically unsuitable for investigating visible persistence. Fehrer and Raab (1962) showed that a stimulus rendered invisible by metacontrast masking could, nevertheless, trigger a rapid response in a simple RT situation. Cumming (1972) and Pollack (1972) showed that stimuli rendered invisible by "sequential blanking" (Mayzner \& Tresselt, 1970) could, nevertheless, be responded to above chance in a choice RT situation. In these two cases, there is a dissociation between the subject's conscious experience and the processes which generate a rapid simple or choice response. The same may be true in visible persistence experiments; a subject may be able to detect that a stimulus has terminated even though a representation of the stimulus is still phenomenologically present. Detection of stimulus offset may trigger an of fset RT, even though the stimulus remains visible. If offset detection lags a little behind stimulus offset with peripheral or with brief stimuli-and both suggestions are plausible-then only peripheral or brief 
stimuli will seem to have brief persistences according to the RT technique. The inverse relationship between intensity and persistence could be due to decreasing latency of offset detection as intensity increases.

Observations reported by Kulikowski and Tolhurst (1973) and Meyer and Maguire (1977) support this argument, since they indicate that a discontinuously illuminated figure can appear to be continuously present (hence, its visible persistence must be longer than its off-time) yet flickering (hence, the subject can tell that it is being presented discontinuously). For example: "At our point of continuitydiscontinuity of grating appearance, there is obvious flicker in the field even though the pattern seems constant" (Meyer \& Maguire, 1977, p. 525). If subjects ever use the neural events responsible for the appearance of flicker as the basis of their responses in the offset-RT condition, then offset-RT is not an appropriate index of the cessation of visible persistence, since the offset response is not triggered by the termination of visible persistence.

Pursuing this line of reasoning, it could be argued that the failure to find any evidence for visible persistence of foveal stimuli of relatively long duration might be due to a very short latency of off-detection responses in the fovea; similarly, if off-detection latency becomes shorter as stimulus duration increases, this would explain the inverse relationship between persistence duration and stimulus duration. Norman and Werblin (1974), investigating the retina of Necturus (mudpuppy), a fish whose retina contains rods and one type of cone, noted that, in response to flashes of light lasting 2 seconds, "the cone response exhibited a fast 'off' component, the rod did not generate any off response but returned only gradually to its dark potential level." Similar results have been described for the retina of the macaque monkey (which is very similar to the human retina) by Whitten and Brown (1973a, 1973b, 1973c).

On the assumption that the onset/offset reaction time technique is a valid one for measuring visible persistence, the results obtained with the technique suggest that longer-lasting stimuli generate visible persistence in the rod system but not the cone system, and that brief foveal or parafoveal stimuli generate a visible persistence whose duration is inversely related to stimulus duration. There are some electrophysiological parallels to these psychophysical results. However, if one wishes to argue that the visible persistence studied by the onset-RT/offset-RT technique is a property of the photoreceptors, one would need to explain away the data offered by Briggs and Kinsbourne (1972) in support of their claim that the visible persistence studied by this technique has a cortical locus.

\section{Stroboscopic Illumination of a Moving Stimulus}

Suppose a stimulus moving from left to right at a rate of $10 \mathrm{in} . / \mathrm{sec}$ is illuminated 10 times per second by an extremely brief flash of light. If the first illumination of the stimulus generates a visible representation which persists for more than 100 msec, then a representation of the stimulus in the first position at which it was illuminated will still be visible when the stimulus is illuminated, 1 in. further on in its trajectory, by the second flash. Thus, when the second flash occurs, two stimuli will be seen, the visible persistence of the first plus the actually illuminated second stimulus. If persistence lasts for less than $200 \mathrm{msec}$, the persistence generated for the stimulus in its first position will no longer be visible when the stimulus is illuminated in its third position; but the second position will still be visible. Thus, under these conditions, what the observer will see is a pair of stimuli moving from left to right, even though only one stimulus is ever physically present. By manipulations of velocity and flash rate, one can estimate the duration of visible persistence.

This effect was thoroughly investigated by Allport $(1966,1970)$; he used a rotating radius rather than a stimulus with a linear trajectory. This was viewed by dark-adapted subjects through a $3.05-\mathrm{mm}$ artificial pupil. The subjects reported how many radii were visible for various flash rates or estimated the angle formed by the sector of radii, and from these reports estimates of the duration of visible persistence could be made. Allport (1970) found that visible persistence time decreased as stimulus luminance increased (from .5 to $25 \mathrm{fL}$ ), a result confirmed by Dixon and Hammond (1972) and Efron and Lee (1971). The technique has also been employed by Wade (1974).

The appearance of two or more radii under these conditions is not due to positive afterimages; this is made clear in the following important passage from Allport (1970, p. 687).

Under certain conditions of low background illumination some much dimmer lines may be seen to persist long after the bright radial fan has passed. After fixating the centre of the display for one or two rotations and then changing fixation, an afterimage of the entire circle filled with stationary lines may persist for several seconds. Reducing the intensity of the stroboscopic flash increases the apparent size of the fan, whereas it decreases the persistence of the much dimmer stationary afterimages. In general the presence of such afterimages (often not detected) was never troublesome except in the particular circumstances of a very low flash rate with zero surround illuminations. Within the fan of simultaneous lines there is no clear ordering in terms of brightness; however, this is an extremely difficult observation to make. At least, what is not seen is a continuous decline in brightness from the leading to the trailing edge of the fan....

Nor can an explanation in terms of the perceptual moment (Shallice, 1964; Stroud, 1948, 1955) be correct. For example, Mollon (Note 1) reported that, if a vertical slit is moved horizontally, and stroboscopically illuminated from behind, and if the top half of 
the slit is viewed through a red filter and the bottom half through a blue filter, more red half-slits than blue half-slits are seen. If persistence were due to discontinuous functioning of the perceptual system, this result would imply that the duration of the perceptual moment could simultaneously have two different values, clearly an unacceptable suggestion. Instead, the conclusion must be that the duration of visible persistence is greater for the red stimulus than for the blue stimulus. This could be an intensity effect or a hue effect, since the two stimuli presumably were not matched for intensity. However, an important point is that the multiple stimuli are colored; this phenomenon is inconsistent with the view that visible persistence is solely a property of the rods, a view entertained by Bartlett, Sticht, and Pease (1968) and by Sakitt $(1975,1976 a)$. If the cones generated no visible persistence, then persisting visible representations would be achromatic; Mollon (Note 1) reported that this was not so. If cones generated briefer visible persistences than rods, Mollon's observers would have seen some colored bars (the most recently illuminated ones) and some achromatic bars; he reported no such result.

It is possible that some form of "filling-in" process was responsible for the finding that all the bars were colored; a way of avoiding this possibility would be to alternate colors in the stroboscopic presentation. If the several bars seen because of visible persistence also alternate in color, it would be difficult to argue that this was because of "filling in." A second reason why this alternating-color experiment is worth doing is that if the phenomenally present bars did alternate in color, this would indicate that no components of the visible persistence were solely mediated by rods: for example, it would be inconsistent with the claim that rod persistence lasts longer than cone persistence, a claim made by Sakitt (e.g., Sakitt, 1975).

Allport described several additional intriguing findings which have been neglected in subsequent discussion of visual persistence. For example, he investigated the effect of increasing the illumination surrounding the black disk upon which the rotating radius was stroboscopically lit. There were 15 subjects in this experiment. Nine of them produced data in which the duration of persistence declined monotonically as surround intensity increased. The remaining 6 subjects produced data in which varying the surround intensity had no effect on duration of persistence. The 6 subjects who showed no surround intensity effect also yielded the six smallest persistence durations at the lowest surround intensity. The latter (6) subjects yielded increased persistence times when loud auditory noise was present; the former (9) subjects yielded decreased persistence with auditory noise.

A second intriguing result is that the difficult technique of counting radii yielded much longer persistence times $(100-300 \mathrm{msec})$ than the easier tech- nique of estimating the angle formed by the sector of radii (30-100 msec).

Allport suggested that both of these results might be explained if one proposed that subjects could exert some control over the duration of visible persistence. As illumination is decreased, visual processing becomes more difficult, and a natural strategy would be to increase persistence duration. This does not, however, assist the subject to resolve radii in the rotating-radius setup, because the different radii are not spatially superimposed. One might argue, then, that nine of Allport's subjects were induced to increase persistence duration as the surround illumination was decreased, this being the natural strategy in response to decreased illumination, while the remaining six did not employ this natural strategy because, unusually, it is maladaptive in the rotating radius situation.

These suggestions of Allport's are intriguing precisely because visible persistence is rarely thought of as a process whose duration is subject to strategic control. However, as he pointed out, studies of timeintensity reciprocity can also be interpreted in this way, since such studies have shown that the more difficult a perceptual task, the longer the "critical duration," the period of time over which there is reciprocity between stimulus duration and stimulus intensity, and this period may be analogous to the duration of visible persistence.

This technique has certain limitations if one wishes to study visible persistence; for example, one cannot measure persistence for long-lasting stimuli, since it is inherent in the technique that the stimuli must be very brief. However, results obtained with this technique agree with those obtained from the other techniques so far discussed; persistence duration increases as stimulus intensity decreases. Allport's clear distinction between visible persistence and afterimage is useful; his discussion of the possibility of subject control over persistence duration is novel and thought-provoking, and Mollon's point that visibly persisting radii are colored is most important.

\section{The Moving-Slit Technique}

If you walk along beside a paling fence and look through it at a scene-let us say a tennis matchyou will find that, at a certain speed of walking, the whole scence appears visible, even though at any one instant you are seeing only a series of vertical strips of the scene, interrupted by the palings. This phenomenon can also be observed if you cut a narrow vertical slit in an opaque piece of paper and move the slit to and fro across a figure underneath the paper. At the right speed, the whole figure can be seen, even though at any one instant only one narrow vertical slice of the figure is visible.

This effect is obviously another form of the rotating-radius effect studied by Allport. Provided the visible persistence of each vertical slice of the 
figure does not terminate before the slit gets back to it, the whole figure will remain continuously visible, even though discontinuously illuminated. The effect has often been studied over the past century; Anstis and Atkinson (1967) review some of the early work. It was claimed by Parks (1965) that the effect occurs even when the consecutive vertical slices of the figure are presented to the same retinal area, as would happen if the eye fixated on the moving slit. If this were so, then the effect could not be built up via visible persistences of adjacent figure segments on adjacent retinal areas. However, Anstis and Atkinson (1967) and Haber and Nathanson (1968) have demonstrated conclusively that the effect occurs only when different slices of the figure are presented to different retinal locations, and hence the visible persistence explanation would appear to be correct.

If so, this pherlomenon provides yet another method for measuring the duration of visible persistence. When the slowest slit-oscillation speed at which the figure is continuously visible is determined, then the time between two consecutive displays of the same slice of the figure is equal to the duration of visible persistence. Haber and Standing (1969), using this method, obtained a value of something over $300 \mathrm{msec}$ for the duration of visible persistence; they also obtained some evidence suggesting that, in this situation, a higher luminance $(50 \mathrm{~mL})$ yields a shorter visible persistence. The moving slit technique has also been used by Stanley and Molloy (1975). In their study, children produced shorter visible persistences than adults, but dyslexic children did not differ from normal children. Since, in other work (Stanley, 1978; Stanley \& Hall, 1973), in which a two-part temporal integration task was used (this task is discussed below), dyslexic children produced longer persistence times than normals, Stanley and Molloy concluded that the moving-slit technique doe not measure the same phenomenon as the temporal integration technique. This remains a problem, all the more so since Arnett and di Lollo (in press) found no relationship between persistence times and reading ability.

The reader is invited to use this moving-slit technique for himself, with a multicolored display. If rod persistence outlasts cone persistence, what should be experienced is that, as the slit-traverse time is reduced, a point will be reached at which the display remains visible as a whole, but achromatically. ${ }^{5}$ My own informal tests have failed to produce this achromatic phase; formal investigation of this issue would be useful.

To summarize: Results obtained using the movingslit technique are consistent with those obtained from other techniques; the slit effect is what would be expected from the existence of visible persistence as revealed by these other techniques, and the finding of an inverse relationship between stimulus intensity and persistence duration, demonstrated using both the synchrony and rotating-radius technique, is also obtained using the moving-slit technique.

\section{Phenomenal Continuity}

When the moving-slit technique is used, a figure is divided up into parts, and each part is displayed discontinuously; furthermore, the different parts are displayed at different times. A minor modification of this technique is to display all the parts simultaneously, but discontinuously; that is, to display the whole figure discontinuously. If visible persistence exists, then the discontinuously presented figure will appear to be continuously present provided its off-time is less than the duration of visible persistence. Thus, one can obtain a measure of the duration of visible persistence by finding the maximum off-time at which a discontinuously presented figure appears to be continuously present; this is the phenomenalcontinuity technique, first used by Haber and Standing (1969) and since then by Meyer and his coworkers (Meyer, 1977; Meyer, Lawson, \& Cohen, 1975; Meyer \& Maguire, 1977).

In Haber and Standing's experiment, there was alternating presentation of a black figure (a circle) and a blank field. The two displays were of equal luminance, and the off-time of the circle was always 20 times longer than its on-time. Cycle time (offtime plus on-time) was varied until the threshold for phenomenal continuity of the circle was reached. Persistence duration was in the range $250-300 \mathrm{msec}$, and was shorter for more intense $(50 \mathrm{~mL})$ stimuli. A second experiment was also carried out using this technique: in this experiment, presentation of the figure alternated between the two eyes in one condition, and was confined to the right eye in another. The dichoptic and monoptic techniques yielded equivalent persistence times. Haber and Standing argued as follows from this result: "Visual persistence is the same whether the repeating stimulation comes in the same eye, or alternates between eyes. This strongly supports a central locus for the effects of persistence and of short-term visual storage." This argument is fallacious. It is based on the reasoning that, if persistence were a peripheral-monocular process, a stimulus would appear to be discontinuous unless, as the moment its persistence in one eye's channel ended, the stimulus were re-presented to the same eye. But obviously the two monocular channels are feeding into a central binocular processor, and if this processor is indifferent to, or ignorant of, which eye is presenting input to it, phenomenal continuity will occur if, at the moment that the persistence of a stimulus in one eye's channel ends, the stimulus is re-presented to either eye. Thus, the finding that per- 
sistence times are the same in the dichoptic and monoptic conditions is not inconsistent with the view that visible persistence is a peripheral-monocular process.

A further difficulty for this experiment is that it contained a number of peculiarities, which were pointed out by Engel (1970). First, when a highluminance stimulus field was used, the interstimulus field was also of high luminance. Thus, the effects of brightness masking were confounded with the effects of stimulus luminance (unnecessarily), and so the finding that the more intense stimulus yielded shorter persistence could be due to the greater severity of masking in this condition. When the interstimulus interval had zero luminance, Haber and Standing found that persistence times were greatly increased. Second, there was an unnecessary confounding between stimulus duration and persistence duration, since the stimulus duration was always $5 \%$ of the total cycle time. Finally, in the dichoptic condition, an adapting field was present for both eyes when the stimulus was absent; but when the stimulus was presented to one eye, the adapting field for the other eye was turned off (see Engel, 1970 , p. 165). As Engel comments: "The reason for using this rather eccentric procedure is obscure. In any case, there is little comment that we can make in explaining the results except to say that the analysis of the visual responses in this situation must be considerably more complicated than Haber and Standing would have us believe. For example, what is the persistence of the response to a blacked out adaptation field? Perhaps the pairing of the circle in one eye and the blacked out adaptation field in the other eye prevented the formation of a binocular response. Or possibly the blackening of the adaptation field cut off the persistence of the previous circle response in the same eye in which case the monocular circle responses would never overlap." Yet another complication is that it is unknown whether, in the monoptic (right eye only) condition, the left eye was blacked out whenever the stimulus was presented to the right eye. If it was not, then obviously the monoptic and dichoptic conditions are not comparable.

A third condition was used in this experiment: monocular presentation with the stimulus duration varying from 4 to $200 \mathrm{msec}$. Persistence time was about $200 \mathrm{msec}$ here, and was independent of stimulus duration. This conflicts with the previous work on the effect of stimulus duration on persistence duration, all of which has indicated that persistence duration decreases as stimulus duration increases, up to a duration of over $100 \mathrm{msec}$; beyond this point, persistence time is independent of stimulus duration. However, as was noted earlier, Bowen, Pola, and Matin (1974) found that the slope of the function relating persistence time to stimulus duration becomes shallower as stimulus luminance decreases; at their lowest luminance, persistence time varied hardly at all as stimulus duration was increased from 50 to $400 \mathrm{msec}$. Unfortunately, Haber and Standing did not report the stimulus luminance they used, so it is not possible to determine with certainty whether this is the explanation of their failure to find any effect of stimulus duration on persistence time. Their use of black-on-white stimuli and reflected rather than transmitted light complicates the situation still further.

The phenomenal-continuity technique has also been used by Meyer, Lawson, and Cohen (1975). The stimulus in their experiment was a vertical or a horizontal square-wave grating of $50 \mathrm{msec}$ duration; subjects adjusted the time-off between successive presentations of the grating until the threshold for apparent continuous presentation of the grating was reached. Adapting luminance was $2.42 \mathrm{fL}$, test stimulus luminance was $1.42 \mathrm{fL}$. The average duration of visible persistence was $177 \mathrm{msec}$. This duration was influenced by orientation-specific adaptation: the visible persistence of a vertical grating, for instance, was briefer when a previously fixated adapting grating was vertical than when it was horizontal. This effect occurred even interocularly, that is, when the adapting grating was presented to one eye and the grating whose persistence was measured was presented to the other eye. Meyer et al. concluded from these results that visible persistence must be a cortical process, since orientation-selectivity and binocularity do not occur in the visual system below the level of the visual cortex: "Duration judgments based on a 'photographic' representation of the retinal mosaic, or on subcortical center-surround receptive fields, should not be influenced by orientation-specific adaptation since that type of effect seems to be restricted to corticl processes." This reasoning suffers from the same difficulty pointed out in earlier in connection with the conclusion drawn by Haber and Standing (1969) from their dichoptic results. Even if visible persistence were an entirely monocular-peripheral effect, it must be analyzed via the cortical visual-information-processing system. Reducing the sensitivity of that system by preadaptation would reduce the visibility of any entirely peripheral visible persistence. Thus, the occurrence of orientation-specific adaptation of visible persistence is not relevant to the question of whether visible persistence is a peripheral or a central process.

In introducing their paper, Meyer et al. argue thus: "adaptation reduced the apparent contrast of target gratings. A lower contrast grating might generate a shorter visual persistence. Increases in stimulus intensity or dark adaptation do increase STVS durations (Haber \& Standing, 1969)"' (p. 569). Now, as has 
already been discussed, Haber and Standing (1969), and numerous others, have shown that increases in stimulus intensity decrease, not increase, the duration of visible persistence. If orientation-specific adaptation mimicked this effect, then such adaptation ought to increase the duration of visible persistence. Instead, such adaptation decreased persistence duration. One might, therefore, even argue that the results of Meyer et al. show, not (as they claimed) that persistence is a central effect, but that it is a peripheral effect, since reducing stimulus intensity at a central level (by orientation-specific adaptation) does not have the same effect as reducing the actual intensity of the stimulus. The discrepancy here could be attributed to the existence of a peripheral monocular visible-persistence process whose duration is inversely related to stimulus intensity, plus the existence of a central binocular system for analyzing visual input (including input from visible persistence), whose sensitivity is reduced by orientation-specific adaptation (via either eye). When sensitivity is thus reduced, the time for which a fading visible persistence remains visible will obviously be shortened; but this is not because the duration of this peripheral process is itself influenced by orientation-specific adaptation.

This work has been followed up by Meyer (1977), who showed that color-specific adaptation of the duration of visible persistence is also obtainable in this situation. Here, however, there was no interocular adaptation effect. This parallels the results of most, but not all, studies of interocular McCollough effects, and it has been speculated (Coltheart, 1973) that the failure to find interocular effects in colorspecific adaptation experiments is because the relevant color-selective cortical cells are all monocular cells, whereas interocular transfer can occur only with binocular cells. In this paper, Meyer notes that the existence of orientation-specific or color-specific adaptation of the duration of visible persistence is compatible with either a cortical locus for persistence or a subcortical locus, or both. The fact that cortical adaptation reduces persistence duration, however, may suggest that persistence is subcortical, as I have argued above.

A third dimension of the visual stimulus, spatial frequency, was used in a phenomenal-continuity experiment by Meyer and Maguire (1977). They simply measured the duration of the visible persistence of vertical square-wave gratings of various spatial frequencies from .9 to 15 cycles/deg; luminance was $10 \mathrm{fL}$. They found that persistence duration increased as spatial frequency increased; the coarsest grating (.9 cycles $/ \mathrm{deg}$ ) yielded a mean persistence of about $300 \mathrm{msec}$, and the finest (15 cycles/deg), a mean persistence of nearly $500 \mathrm{msec}$. Meyer and Maguire suggested that this interesting result was also unexpected: "It was not predicted a priori that STVS duration would increase as the target consisted of finer and finer lines. In fact, the result was slightly counterintuitive because the finer (higher spatial frequency) lines were harder to see, but this 'harderto-see' percept lasted much longer." However, this is the expected, not the counterintuitive, result. When the processing of a target is made more difficult (by reducing its contrast or its duration, or by increasing the difficulty of the judgement required of the subject), it has always been found that the duration of the target's visible persistence increases. Thus, making the processing of a grating more difficult by increasing its spatial frequency ought to, and did, increase the duration of its visible persistence. However, a simpler explanation of this finding, which does not have recourse to the perhaps rather nebulous concept of "difficulty of processing," will be discussed below (in the section on sustained and transient channels).

The results obtained using the phenomenalcontinuity technique yet again indicate that visible persistence duration is increased by reducing stimulus luminance. One new finding obtained with this technique is that, when the stimulus is a square-wave grating, persistence duration depends strongly on the spatial frequency of the grating, higher frequencies generating longer persistences.

\section{Temporal Integration of Form Parts}

This technique is a variant of the moving-slit and phenomenal-continuity techniques. It was introduced by Eriksen and Collins $(1967,1968)$. They used as a stimulus a CVC nonsense syllable whose letters were made up of dotted lines. When the set of dotted lines making up the CVC was divided into two stimuli, $S_{1}$ and $S_{2}$, by assigning dots at random to $S_{1}$ or $S_{2}$, the results was two dot patterns which contained no usable letter information. Only when $S_{1}$ and $S_{2}$ were superimposed could any of the letters be identified. It is obvious how these stimuli may be used to study visible persistence: present $S_{1}$, and then, after some interstimulus interval (ISI), present $S_{2}$. If the ISI is shorter than the visible persistence of $S_{1}$, then $S_{2}$ will be superimposed upon $S_{1}$ and the CVC stimulus will be identifiable. If the ISI is longer than the visible persistence of $S_{1}$, then the subject will not be able to identify the three letters. Thus, the maximum ISI at which the three letters can be identified is the maximum duration of the visible persistence of $S_{1}$. Eriksen and Collins (1967) found that performance was still above chance for a 6-msec $S_{1}$ at $5 \mathrm{~mL}$ when ISI was $100 \mathrm{msec}$. They varied the intensity of $S_{1}$ and $S_{2}$, orthogonally, without obtaining clear evidence of the relationship between intensity and persistence. Cohene (1975), using this technique, varied the durations of $S_{1}$ and $S_{2}$, keeping the two durations equal to each other. As duration increased, performance grew worse.

The technique was used more systematically by Pollack (1973). He showed that the ability of a subject to perform a task which could only be done by 
integrating the sequentially displayed stimulus halves $S_{1}$ and $S_{2}$ was inversely related to the brightness of $S_{1}$ and also inversely related to the brightness of $S_{2}$. The first of these findings is consistent with the various results showing an inverse relationship between stimulus intensity and duration of persistence, discussed earlier. The second finding, however, cannot be explained in this way. Pollack reiterated the suggestion by Eriksen and Collins $(1967,1968)$ that there exist discontinuity detectors which disrupt the integration of $S_{1}$ and $S_{2}$; the greater the brightness of $S_{2}$ relative to $S_{1}$, the more severe this discontinuity would be, and hence the more difficult integration of $S_{1}$ and $S_{2}$ might be.

This technique was next used in an elegant series of investigations by Di Lollo and his co-workers (Di Lollo, 1977, 1978, in press; Di Lollo \& Wilson, 1978; Hogben \& Di Lollo, 1974), who divided a figure into not 2 but 24 parts, each part displayed at a different point in time. These figure parts were actually dots, each displayed extremely briefly $(50 \mu \mathrm{sec})$ and each occupying a different cell in a $5 \times 5$ square matrix of locations. One location was unfilled. The subjects' task was simply to specify the coordinates of the unfilled location.

At interdot intervals of about $3 \mathrm{msec}$ or so-that is, with a total display time of about $80 \mathrm{msec}$, or less-the 24 dots appeared to be simultaneously present, and performance in identifying the unfilled location was virtually perfect. However, as the interdot interval, and hence the total display duration, increased further, some impression of sequential occurrence of the dots was introduced, and errors in identifying the unfilled location began to occur. The relationship between percent errors and total display duration was ogival in shape, with average error rate increasing from near zero at a display duration of $80 \mathrm{msec}$ to about $70 \%$ at a display duration of $280 \mathrm{msec}$.

At the longer durations, subjects reported that there appeared to be more than one missing dot in the display. Hogben and Di Lollo inferred that this was because at long display durations the visible persistence of one or more of the earlier dots had terminated by the time that last dot was presented in the display sequence. They confirmed this by showing that the earlier a dot was presented in the display sequence, the more likely it was that this dot's location would be nominated by the subject as having been the unfilled location. Locations where dots had been presented within $120 \mathrm{msec}$ of the last dot were rarely the subject of wrong responses. This implies that the minimum duration of visible persistence in this situation is $120 \mathrm{msec}$.

When the first 6 dots were plotted at a higher intensity than the subsequent 18 , this neither improved nor harmed performance. One might have expected poorer performance on the principle that increasing intensity reduces the duration of visible persistence.
Next, Hogben and Di Lollo investigated the effect of departure from regularity of timing of the sequence of dots: they presented the first 6 dots in $7 \mathrm{msec}$, and, after a variable ISI, the remaining 18 dots in $23 \mathrm{msec}$. Performance was considerably worse in this condition than it was when the dots were presented at equally spaced points in time. Subjects reported that this was because they found great difficulty in integrating the first 6 dots with the following 18 dots. The two dot bursts were seen as separate perceptual events; the first 6 dots were seen clearly but briefly, and were entirely obliterated by the subsequent burst of 18 dots. The mechanism responsible for this effect could be the discontinuity detector, discussed above in relation to the work of Eriksen and Collins $(1967,1968)$ and Pollack (1973), the discontinuity in Hogben and Di Lollo's situation being a temporal discontinuity.

This task was further explored by Di Lollo (1977). In his experiment, 12 dots were plotted effectively simultaneously, and remained on view continuously, for a period that varied from 10 to $200 \mathrm{msec}$; then there was a blank period of $10 \mathrm{msec}$; finally, the remaining 12 dots were plotted, effectively simultaneously, for a period of $10 \mathrm{msec}$. Performance was almost perfect when the display duration of the first set of 12 dots was $80 \mathrm{msec}$ or less. At $120 \mathrm{msec}$, performance was less than perfect; and performance was not much better than chance at $200 \mathrm{msec}$.

Di Lollo (1977) carried out a second experiment, this time using a sequential display of dots. Here the onsets of the 24 dots occurred at regular intervals (1 dot/10 msec). In the control condition, each dot lasted for $1.5 \mu \mathrm{sec}$. In the experimental condition, this was true for all dots but the "12th," which lasted for $100 \mathrm{msec}$. Its offset was midway between the offsets of the 11 th and the 13th dots. The 12th dot's position was chosen as having been unfilled far more often in the experimental condition than in the control condition. At each of the other 23 positions in the temporal sequence of dots, the two conditions were equal to each other. Di Lollo suggested that this was because the 12th dot did not persist for as long after its offset as did the other, briefly presented, dots. Thus, when the subject was making his judgment, the visible persistence of the 12 th dot would often have terminated even though dots whose offsets preceded the offset of the 12th might still be visibly persisting. The long duration of the 12 th dot cannot have induced a discontinuity detection effect at the dot's offset, since dots whose offsets (or onsets) preceded that of the 12th were not more difficult to process in the experimental than in the control condition.

This result, then, appears to be due to the existence of the inverse relation between stimulus duration and duration of visible persistence. The 12th dot's location was chosen as the location of the 
missing dot so often because the 12th dot's visible persistence, measured from its offset, was so brief that this dot was often no longer visible at the point when the subject was making his decision.

Not only did Di Lollo offer this explanation of his results; he also suggested a reason for the existence of the inverse relation between stimulus duration and visible persistence. This was that visible persistence was generated by, and so should be measured from, the onset of a stimulus not its offset. If visible persistence lasts for a constant time from stimulus onset, then obviously there will be an inverse relationship between stimulus duration and persistence time measured from offset. (What is more, the function relating the two will have a slope of -1.0 ; this has sometimes, but not always, been found, as discussed earlier.)

This cannot be true in precisely the form stated by Di Lollo. In his experiment, the onsets of the 12th dot and the 2 nd dot (in the experimental condition) were simultaneous. If visible persistence begins at stimulus onset and lasts for a constant amount of time, regardless of stimulus duration, then the offset of the visible persistences of the 2 nd and 12th dots should, on average, be simultaneous. Therefore, the subject should be just as likely to choose, as the location of the missing dot, each of these two dots' locations. This did not happen. The 2 nd dot's location was chosen much more often than the 12th's, indicating that, despite the simultaneity of onset of these two dots, the visible persistence of the 2 nd dot usually terminated earlier than the visible persistence of the 12th. This point is discussed by Di Lollo (in press).

If the time between stimulus onset and persistence offset is a constant, then the function relating stimulus duration to the duration of visible persistence as measured from stimulus offset will have a slope of -1.0 . Another way of putting this is that stimulus duration plus duration of persistence (measured from offset) will be a constant. Di Lollo's data depart from this; the sum of stimulus duration plus duration of persistence measured from stimulus of fset is not constant, but is larger for the longer stimulus. There are two possible reasons for this (which cannot be distinguished unless several stimulus durations are used). The first is that persistence should be measured from stimulus offset, and that there is a decreasing negatively accelerated function relating stimulus duration to persistence duration. The second is that there are actually two persistences, one generated by stimulus onset and the other generated by stimulus offset; their durations are both independent of the stimulus duration, but the onset persistence is, on the average, longer lasting than the offset persistence. Either suggestion is consistent with Di Lollo's data. The view that persistence is of fixed duration and generated at stimulus onset is, however, not entirely consistent with his data.
Di Lollo and Wilson (1978) have demonstrated an effect similar to that shown by Di Lollo (1977). The 24 dots were divided into three groups: Group B (6 dots displayed for $10 \mathrm{msec})$, Group C (12 dots displayed for $10 \mathrm{msec}$, beginning $20 \mathrm{msec}$ after the end of Group B), and Group A (6 dots displayed for a variable period, from 20 to $200 \mathrm{msec}$ ). The offset of Group A was always $10 \mathrm{msec}$ after the offset of Group B and $10 \mathrm{msec}$ before the offset of Group C. The location named as empty was virtually never one which had been occupied by a Group B or Group C dot; but it was sometimes a location occupied by a Group A dot, and the likelihood of this increased as the duration of the Group A display was increased. This is qualitatively consistent with the idea that persistence lasts for a constant time after stimulus onset. However, even with an A duration of $200 \mathrm{msec}$, subjects were far better than chance at the task; if the maximum duration of visible persistence were less than $200 \mathrm{msec}$, and if persistence began at stimulus onset, then all 6 of the Group A dots should be invisible by the end of the A display, and the subject would have no way of selecting the actual unfilled location from the seven locations not visibly containing dots.

This work has been further pursued by Di Lollo (in press). In one experiment, the 24 elements of the $5 \times 5$ matrix with one element missing were not dots, but triangles, each defined by three dots, one per apex. If the triangles representing the six Group $A$ stimuli were inverted $10 \mathrm{msec}$ before the offset of the Group A stimuli, then performance was almost perfect, even for long durations of Group A, which without the inversion would lead to poor performance. Thus, as Di Lollo says, "a change in local detail (rotated condition) generated a new lease of persistence which enabled perceptual bridging of the temporal gap despite the unchanged overall configuration of the leading display. This permitted the inference that visible persistence was an attribute of local detail rather than of the overall configuration."

Di Lollo also showed that the identifiability of a brief $(20 \mathrm{msec})$ single alphabetic character increased monotonically with the duration of a preceding masking stimulus as the mask duration increased from 20 to $640 \mathrm{msec}$. Di Lollo proposed that this was because increasing the mask duration reduced the duration of the mask's persistence and that it is this persistence which interferes with identification of the aftercoming target, that is, causes forward masking. Subjective reports of observers supported this: As mask duration increased, the time by which the target outlasted the mask appeared to increase. This led Di Lollo to present target and mask concurrently, that is, to have their of fsets simultaneous and to investigate the effects of mask duration; and he was able to show that, as mask duration increased, percent correct identification of the target did also, even though the mask was always present when the 
target was present. This pattern of results illustrates the inverse relationship between the duration of a stimulus and the duration of visible persistence.

Several of the properties of visible persistence demonstrated via other techniques have thus also emerged from experiments using the temporal integration technique. Visible persistence, as measured by this technique, is briefer for more intense stimuli [though Hogben and Di Lollo (1974) did not observe this effect] and also briefer for longer lasting stimuli. A phenomenon which is unique to this method is that of disruption by discontinuity detection: Integration of one visual stimulus with the visible persistence generated by an earlier stimulus is made more difficult as the spatial or temporal properties of the two stimuli become more different. This suggests that the process of integration is carried out by a rather sophisticated stage of the visual system; but this, of course, does not imply that visible persistence itself cannot be a property of some much earlier and much simpler stage.

\section{Stereoscopic Persistence}

The existence of stereoscopic persistence has been most elegantly investigated by Engel (1970), whose work has been ignored in many discussions of visible persistence and iconic memory. Engel used, as stimuli, pairs of Julesz random-brightness fields. Each pair consisted of two square matrices containining 10,000 brightness elements. Each element was randomly assigned one of seven possible brightness values. The brightness distributions in the two matrices of a pair were identical, except that a central square submatrix was displaced laterally in one matrix relative to the other. Thus, when either matrix is inspected, no contours within the matrix are evident: a two-dimensional gray speckled surface is seen. However, when the two matrices of a pair are presented, one to each eye, and stereoscopically fused, stereoscopic depth is experienced: the square submatrix is seen at a different depth than the surround.

It is known that if one member of a stereo pair is presented briefly to one eye and then, after a short delay, the other member of the pair to the other eye, stereopsis may still be experienced if the interstimulus interval is not too long (e.g., Efron, 1957; Ogle, 1963). This has been explained in terms of visual persistence: the first stimulus persists in its monocular channel long enough for its persistence to be simultaneous with the presentation of the second stimulus, and this temporal overlap of the two monocular stimuli permits steropsis to occur.

One of Engel's contributions was to appreciate the distinction between (1) how long a monocular stimulus will persist-that is, what the maximum interstimulus interval is at which stereopsis still is experienced with asynchronous presentation of the two members of the stereo pair; and (2) how long the stereoscopic sensation itself persists, once it is generated.

This distinction can be investigated experimentally. Let $\mathbf{R}$ be the right-eye member of a stereo pair, and $L$ be the left-eye member. Assume each stimulus is presented very briefly (say, $1 \mathrm{msec}$ ). The duration of monocular persistence can be measured by finding the maximum interval between $R$ and $L$ at which stereopsis is experienced. The duration of the stereoscopic sensation can be measured by generating stereopsis with a presentation of $\mathrm{R}$ and $\mathrm{L}$, and finding the maximum interval for which one can wait before presenting $R$ and $L$ again, without causing an interruption of the stereoscopic sensation.

Thus, a sequence of alternating $R$ and $L$ stimulation is to be delivered to the observer. Engel used the symbol $t$ to refer to the interval between two consecutive stimulations of the same eye, and the symbol $S$ to refer to the shorter of the two possible time delays between a stimulus presentation to one eye and a stimulus presentation to the other eye. The maximum value of $S$ at which any stereopsis is still experienced is the maximum value of monocular persistence. For any value of $S$ which permits stereopsis to be experienced, the maximum value of $\mathrm{t}$ at which depth is perceived continuously, that is, the stereoscopic sensation is always present, is the maximum value of stereoscopic persistence. Independent manipulation of $S$ and $t$ thus allows independent investigation of the two forms of persistence; and this is what Engel did.

One possibility is that there is no such thing as stereoscopic persistence. If so, stereopsis will only be experienced while there are simultaneous monocular inputs to the stereopsis mechanism (these inputs being produced either from the stimulus or from its monocular persistence). In this case, if $S$ is set at the maximum value at which stereopsis can be experienced, that is, at the point at which the leading eye's persistence just lasts long enough to be present at the onset of the trailing eye's stimulus, then only one monocular input will be available after the offset of the trailing eye's stimulus (namely, the trailing eye's monocular persistence) and so stereopsis will not be experienced after the offset of the trailing eye. Thus, the stereoscopic sensation will be discontinuous unless the leading-eye stimulus is presented at, or before, the point in time at which its monocular persistence terminates. Put another way, this means that, at the maximum value of $S$ at which stereopsis can be experienced, if the stereopsis is to be seen as continuous, both members of the stereoscopic pair would need to be presented: one to generate the stereopsis and the other to generate its monocular persistence. Engel showed that this was not the case. At the maximum value of $\mathrm{S}$ sufficient for stereopsis, presentation of only the second member of the stereo 
pair would still yield a continuous sensation of stereopsis, and even if there was a subsequent time period before the first member of the pair was represented, stereopsis could be seen continuously. Therefore, a stereoscopic persistence must exist independently of persistence in monocular input channels.

Engel measured properties of these two persistences. He showed that the duration of monocular persistence (the maximum intereye interval at which any stereopsis was perceived) was an increasing negatively accelerated function of stimulus duration; it was equal to about $20 \mathrm{msec}$ for stimuli of $1 \mathrm{msec}$, and increased to about $80 \mathrm{msec}$ for stimuli of $10 \mathrm{msec}$ duration. At such short durations, it is more appropriate to refer to stimulus energy than to stimulus duration. Thus, the results show that, within a particular energy range ( $6 \mathrm{fL}$ presented via an artificial pupil for durations from 1 to $10 \mathrm{msec}$ ), the duration of monocular persistence is an increasing negatively accelerated function of stimulus energy. Engel's Figure 6 suggests that the asymptotic duration of monocular persistence is, in fact, about $80 \mathrm{msec}$. It should be noted that his stimuli were all of low energy, so that it is an open question whether the inverse relationship between stimulus intensity and persistence might not hold in his experimental situation when high-energy stimuli are used.

The maximum duration of stereoscopic persistence was much longer than the maximum duration of monocular persistence. The duration of stereoscopic persistence depended upon the duration of monocular overlap, that is, the period of time for which the monocular stimulus or its persistence was present simultaneously with the other monocular stimulus or its persistence. This overlap had to be at least $4 \mathrm{msec}$ for any stereopsis to occur at all; and as the duration of the overlap increased to about $35 \mathrm{msec}$, the duration of stereoscopic persistence increased to about $300 \mathrm{msec}$. This appears to be an asymptote of the function relating the duration of stereoscopic persistence to the duration of the monocular overlap generating the stereoscopic response.

When stimulus intensity and stimulus duration were jointly varied, the duration of the stereoscopic persistence was an increasing negatively accelerated function of the product of these two variables, indicating that stimulus energy was the determining factor.

Engel also showed that, for a fixed duration of monocular response overlap, the duration of stereoscopic persistence increased as a function of stimulus energy. Thus, there must be two properties of monocular inputs which influence the duration of the persistence of the stereoscopic sensation generated by these inputs: the duration for which they are concurrent (i.e., the duration of monocular response overlap) and their "amplitudes," where it is assumed that monocular response amplitude is an increasing func- tion of stimulus energy. Thus, an increase in stimulus energy prolongs the duration of stereoscopic persistence in two ways: It increases the duration of the monocular responses (thereby increasing the duration of monocular response overlap) and it increases the amplitude of the monocular response.

The manner in which monocular amplitude and monocular overlap combine in determining stereoscopic persistence remains to be determined. The simplest possibility is that the stereoscopic response is a result of the summation of monocular amplitudes over the duration of the monocular response overlap. Engel showed that his data were inconsistent with this proposal.

The results of his work may be summarized conveniently as follows. In order to generate a stereoscopic sensation, the second member of a stereo pair must be presented within about $80 \mathrm{msec}$ of the offset of the first member. However, once a stereoscopic sensation has been generated, one can wait up to about $300 \mathrm{msec}$ before re-presenting the two members of the pair again, without causing an intermittency in the stereoscopic sensation. Thus, two forms of persistence exist here: persistence in the monocular channels serving as input to the mechanisms for stereopsis, and persistence of the binocular system responsible for sensation itself. In addition, it so happens that the binocular persistence lasts much longer than the monocular persistence.

As has been pointed out earlier in this paper, various attempts have been made at deciding whether visible persistence is a peripheral or a central process: Bartlett, Sticht, and Pease (1968); Briggs and Kinsbourne (1972); Haber and Standing (1969); Meyer, Lawson, and Cohen (1975). It was also pointed out that none of these attempts were satisfactory, for a variety of reasons. However, it is quite clear from Engel's work that there is a form of visible persistence which can only be central, since it is a property of a binocular stage of the visual system: the stage responsible for the stereoscopic sensation.

The only aspect of Engel's work which is not entirely clear is the status of the monocular response persistence whose existence he demonstrated. This persistence is definitely visual, since it is a property of one stage of the visual system; but is it visible? The method by which Engel investigated it does not require that it be visible, whereas the method by which he investigated stereoscopic persistence did depend upon the latter's visibility. Engel did not deal with this question at any length, merely pointing out that, at longer values of $t$ (within-eye repetition interval), the stereoscopic sensation was continuous but illumination appeared to be flickering; and if the observer closed one eye, there was obvious temporal discontinuity of the stimulus viewed by the other eye. This shows that the duration of the visible persistence of 
monocular input is shorter than stereoscopic persistence. Engel's measurements demonstrated that the duration of the visual persistence of the monocular channels feeding into the binocular stereopsis system is shorter than stereoscopic persistence. These two results are compatible with, but certainly do not demand, the view that one can identify these two forms of monocular persistence. Thus, it is an open question whether Engel was investigating visible persistence when measuring the persistence of the monocular channels which provide input to the stereopsis system. I shall argue that he was not, that is, that the monocular systems which possess the property of persistence and which provide input to the stereopsis system are not those responsible for sensations of visible persistence.

There are two reasons for this claim. The first stems from results reported by Ross and Hogben (1974), who used a new method of stimulus presentation, the "stochastic dot stereogram." The observer views two oscilloscope screens, one for each eye, binocularly fused. Under computer control, a point on one screen is chosen randomly and a dot is presented there for a few microseconds. Then, $150 \mu \mathrm{sec}$ later, a dot is plotted for a few microseconds in the same position on the other eye's oscilloscope screen. After a delay of $150 \mu \mathrm{sec}$, another pair of points is plotted in the same way. This sequence of pair plotting proceeds continuously.

In order to introduce a binocular disparity, a square area in the center of the display is defined. Any point destined to be plotted within this area is displaced to the left on one eye's screen and to the right on the other eye's screen. Under these conditions, the observer sees a square containing moving points, at a different depth from a surround, also containing moving points. Thus, despite the fact that at any instant the observer is being presented with at most only one dot, and that only to one eye, stereopsis is apparent. Thus, some form of visual persistence must be operating. Ross and Hogben investigated it by introducing a delay between the eyes. A point destined for the trailing eye had to wait for a period of from 0 to $108 \mathrm{msec}$ before it was presented to that eye. Lags of up to $38 \mathrm{msec}$ had no detectable effect on stereopsis, but at longer lags performance of a task requiring stereopsis began to be imperfect, though it was still somewhat better than chance at the longest lag, $108 \mathrm{msec}$. Performance was at chance with a lag of $150 \mathrm{msec}$. Thus, the lower and upper bounds for monocular response persistence duration were 36 and $150 \mathrm{msec}$, which agrees adequately with Engel's average value, $80 \mathrm{msec}$. Hogben and Ross tried two (unspecified) stimulus energy levels without being able to detect any effect of intensity on monocular response persistence duration; if the lower of these two levels was approximately equal to the highest level used by Engel, then his conclusion that the function relating monocular persistence duration to stimulus energy was asymptoting at his highest energy level would be confirmed by Hogben and Ross's findings.

The monocular visual persistence of points in these displays, as indexed by the relationship between lag and stereoscopic performance, begins to deteriorate at about $36 \mathrm{msec}$ after stimulus onset; $120 \mathrm{msec}$ after onset it is so poor that stereoscopic performance is severely impaired. Yet Hogben and Ross note the following: "Under stochastic presentation conditions, the field of view is filled with points all of identical ${ }^{6}$ brightness and quality. The number visible at any one time is, to a good approximation, the number plotted in $100-120 \mathrm{msec}$. Points, that is to say, remain visible for up to $120 \mathrm{msec}$, a fact consistent with most findings with pattern perception and especially the findings of ... Hogben and Di Lollo (1974)." Thus, as Hogben and Ross point out, there is a large discrepancy between the persistence times of the monocular channels feeding into the stereopsis system and the persistence times of the channels (wherever they are located) responsible for apparent simultaneity of sequentially presented items, that is, for what I have been calling visible persistence. Thus, the monocular persistence studied by Hogben and Ross is not the visible persistence studied by the methods described earlier in this chapter.

A second reason for distinguishing between the two forms of persistence is that Engel's monocular persistence increases in duration as stimulus energy increases, whereas the visible persistence discussed throughout this chapter decreases in duration as stimulus energy increases.

Thus, although the monocular channels which feed into the stereopsis mechanism do exhibit persistence, and this persistence is by definition visual, there is no reason to describe it as visible or to believe that it plays any part in investigations of visible persistence except for its role in stereoscopic persistence.

The last study of stereoscopic visible persistence to be discussed here is that of Fox, Lehmkuhle, and Shea (Note 2). They used a dynamic random-dot stereogram technique to present $5 \times 3$ arrays of letters which were not discriminable with monocular vision. Following the technique introduced by Sperling (1960), one of the three rows of letters was cued for report, after stimulus offset. The existence of a rapidly decaying visual memory would be implied by the superiority of partial-report performance over fullreport performance. Fox et al. found no difference between the two types of performance, and hence concluded that stereoscopic letters do not generate iconic memory. This result suffers from the difficulty that the task of identifying stereoscopic letters was very time-consuming: "When 15 stereo forms are 
presented simultaneously, the forms are quickly seen in depth but the configuration of contours, which identify them as unique letters, is not immediately apparent. Rather, they appear as blobs that must be scrutinized before correct identification is possible." At stimulus offset, the subject must first decode the partial report cue and decide which row has been cued, a process which takes time (Averbach \& Coriell, 1961); then, having identified the cued row, he must identify its five letters, which is evidently a very slow process in the stereoscopic situation. While all this was going on, any stereoscopic iconic memory will be decaying away. Thus, the amount of information which could be extracted from such a memory might be so small as not to be evident in a comparison of partial report with full report performance.

The results of work on stereoscopic persistence are thus relatively straightforward. Persistence is a property of the monocular channels which provide input to the stereopsis system, so that stereopsis can occur even with asynchronous presentation of stereo pairs; but there is no reason to believe that this form of persistence is visible. The stereoscopic sensation, however, once generated, persists visibly for up to $300 \mathrm{msec}$. This is indubitably a cortical form of persistence, so its existence refutes any claims to the effect that all forms of visible persistence are subcortical (e.g., retinal).

\section{Stimulus Luminance, Retinal Illumination, and Rod Saturation}

It was pointed out earlier that Sakitt (1976b) had obtained increasing durations of visible persistence when stimulus intensity was increased, whereas all the other studies reviewed here in which stimulus intensity was varied obtained an inverse relationship between stimulus intensity and persistence duration. It was suggested that this was because Sakitt had used much more intense stimuli than had any of the other investigators. A direct comparison is not easy, however, since Sakitt refers to her stimulus intensities in terms of trolands (i.e., in terms of retinal illumination), whereas most of the other studies described here specify stimulus intensity in terms of luminance. A brief comment on illumination and luminance is therefore necessary, especially since a concept which may be of importance here-rod saturation-is a function of retinal illumination rather than stimulus luminance.

The amount of light which actually reaches the retina depends upon two factors: the luminance of the stim: '"s and the area of the pupil. As pupil area increases, retinal illumination increases, even with a stimulus of fixed luminance.

Stimulus luminance is measured in terms of footlamberts, millilamberts, or candelas per square meter. They may be converted to each other as follows: $1 \mathrm{fL}=1.0763 \mathrm{~mL}=3.426 \mathrm{~cd} / \mathrm{m}^{2}$.
Retinal illumination is measured in trolands, and is given by the product of stimulus luminance (in candelas per square meter) and pupil area (in square millimeters) (Westheimer, 1966).

Thus, if stimulus luminance is specified, and one wishes to estimate retinal illumination, it is necessary to know what the pupil area is. When an artificial pupil is used, this is simple; when, as usual, the natural pupil is used, the matter is more complicated. The size of the pupil will depend upon the luminance of adapting stimulation. Le Grand (1957) provides a formula for estimating pupil diameter (in millimeters) as a function of the mean luminance $L$ of the visual field (in $\mathrm{cd} / \mathrm{m}^{2}$ ). It is:

$$
d=5-3 \tanh \left(.4 \log _{10} L\right)
$$

The minimum pupil diameter is about $2 \mathrm{~mm}$ and the maximum about $8 \mathrm{~mm}$ (Geldard, 1953); these diameters correspond to pupil areas of $3.14 \mathrm{~mm}^{2}$ and $50.26 \mathrm{~mm}^{2}$. As a rough guide, Le Grand's formula indicates the following: With an adapting field of $1 \mathrm{fL}$, pupil diameter is $4.35 \mathrm{~mm}$ (area $14.83 \mathrm{~mm}^{2}$ ); at $10 \mathrm{fL}$, the diameter is $3.36 \mathrm{~mm}$ (area $8.86 \mathrm{~mm}^{2}$ ); at $50 \mathrm{fL}$, the diameter is $2.86 \mathrm{~mm}$ (area $6.43 \mathrm{~mm}^{2}$ ); and at $100 \mathrm{fL}$, the diameter is $2.70 \mathrm{~mm}$ (area $5.72 \mathrm{~mm}^{2}$ ).

Now, according to Sakitt (1976b), stimulus intensity began to have an effect on persistence duration at a retinal illumination of $3.16 \mathrm{log} \mathrm{Td}$. With a maximum possible pupil area, this corresponds to about $8.4 \mathrm{fL}$, or $7.8 \mathrm{~mL}$. If the pupil is not fully dilated, higher luminances would be required to reach this level of retinal illumination. Referring back to the various studies of visible persistence described earlier, and taking into account the use of artificial pupils in several of them and of light-adapted subjects in others, these calculations indicate that a retinal illuminance of $3 \log \mathrm{Td}$ was not approached in any of these studies except in some of the conditions of two of the studies using the reaction time technique (Pease \& Sticht, 1965; Rains, 1961).

To consider now the phenomenon of rod saturation: at a retinal illumination of about $3 \log \mathrm{Td}$ or higher, rod output is at its maximum; further increases in retinal illumination do not produce further increases in rod output. The rods are thus said to be "saturated" at this illumination (Aguilar \& Stiles, 1954; Sakitt, 1976b). This concept is considered further below, since it is central to analyses of visible persistence and iconic memory proposed by Sakitt; for the moment, it suffices to note that $3 \log \mathrm{Td}$ corresponds to $51 \mathrm{fL}$ with a 2.70 -mm-diam pupil (as produced by an adapting field of $100 \mathrm{fL}$ ) and to $5.8 \mathrm{fL}$ with an 8-mm-diam pupil. These values are useful when considering whether rod saturation is likely to have been attained in any particular tachistoscopic experiment. 


\section{Visible Persistence: A Summary}

The results of the numerous studies of visible persistence reviewed here can be summarized as follows. When a visual stimulus whose retinal illumination is less than 1,000 $\mathrm{Td}$ is presented for a duration of up to about $150-200 \mathrm{msec}$, the stimulus remains visible for some time after its physical offset. The duration for which this visible persistence lasts is inversely related to the duration of the stimulus. The function relating the duration of such persistence to the duration of the stimulus has a slope which changes from near zero (i.e., no effect of stimulus duration) to about -1.0 as stimulus luminance is increased. The slope of -1.0 is attained at quite low luminances, and is not changed by further increases in luminance. This particular value for the slope is an important one, since a slope of -1.0 means that the interval between stimulus onset and persistence of fset is constant, regardless of where, within this interval, the offset of the stimulus is located. Such constancy invites some interesting claims: one is Efron's, that there exists a minimum duration for a perception, and another is Di Lollo's, that persistence is generated by, and so should be measured from, the onset of the stimulus, not its offset. The latter claim, however, does not agree exactly with all of the results obtained by Bowen et al. (1974), or with Di Lollo's own results.

The visible persistence of relatively brief stimuli lasts for a time that is inversely related to stimulus luminance, as well as being inversely related to stimulus duration. There is perfect reciprocity between these variables up to a duration of $100 \mathrm{msec}$, or a luminance of $2.8 \mathrm{fL}$. Beyond this point, duration exerts a stronger effect than luminance. This inverse relationship is not observed when retinal illumination is high (greater than 1,000 Td): here increases in illumination produce increases in the duration of persistence (Sakitt, 1976b).

The observation by Mollon (Note 1) that the multiple persistences visible when a single moving stimulus is stroboscopically illuminated by colored light are not only visible but colored implies that the cones are involved in visible persistence; if every one of the visible persistences in this situation is colored, this would imply not only that the cones persist, but that their persistence times are no shorter than those of rods. The question of rods vs. cones is discussed further below.

When the stimulus whose persistence is visible is a square-wave grating, the duration of this persistence is powerfully influenced by the spatial frequency of the grating, higher frequencies producing longer persistences. When a stereoscopic form is made visible by presenting two monocular stimuli neither of which contain form information, the stereoscopic sensation not only persists, but persists for much longer than does the monocular information. When two stimuli are presented at different times to similar retinal locations, the visible persistence of the first can be integrated with the second. Spatial or temporal dissimilarities between the two stimuli can impair such integration, perhaps through the operation of a "discontinuity detector."'

An unsettled issue is whether relatively long-lasting stimuli have any visible persistence at all. Experiments using the synchrony-judgment technique find such persistence when test and probe stimuli are in the same modality, but none when they are in different modalities. Experiments using the technique of RT to onset and offset have obtained results suggesting that long stimuli do not have any persistence when presented foveally, but do when presented peripherally.

\section{THE RELATIONSHIP BETWEEN VISIBLE PERSISTENCE AND ICONIC MEMORY}

As pointed out at the beginning of this paper, those who have considered how visible persistence is related to iconic memory have, in general, regarded the two entities as identical. Julesz and Chiarucci (1973) claimed that visible persistence as studied using the method of temporal integration of form parts (Eriksen \& Collins, 1967, 1968) was the same thing as iconic memory studied using the partial-report technique. Haber and Standing (1970) were particularly forthright in identifying visible persistence (as studied by the synchrony-judgment technique) with iconic memory (as studied by partial report): "The interclick interval is a reliable and useful indicator of the visual persistence of a flash ... it yields data of the same high order of reliability and magnitude found by the far more laborious and indirect procedures of Sperling (1960), Averbach and Coriell (1961), and others.... These estimates are based on how long the onset of an indicator can be delayed after a display has been terminated while still permitting the subject to identify the item in the display .... [Our] experiments provide more direct evidence that the visual storage assumed to underlie information extraction is in fact visual and available to the subject for his direct estimation."

Similar views have been taken by Turvey (1978), who refers to "a very special kind of visual representation that we have chosen to call 'iconic.' A more significant feature of this representation is that it is visible" "; and by Sakitt [e.g., "the existence of iconic memory for form can also be demonstrated with the successive-field paradigm (Eriksen \& Collins, 1967, 1968; Sakitt \& Long, 1978) and by the subjective persistence of the icon (Sperling, 1967; Sakitt, 1975, 1976a, 1976b)" (Sakitt \& Appelman, 1978, p. 566)].

In part, this is a question of definition. If the successive-field or subjective-persistence techniques 
depend upon visual process $\mathrm{X}$ and the partial-report technique depends on a different visual process $Y$, then we can call process $X$ "iconic memory" and process $Y$ something else, or call process $Y$ "iconic memory" and process $X$ something else. What we cannot do without creating the utmost chaos is to use the term "iconic memory" to refer both to $X$ and to $\mathrm{Y}$ if it is indeed the case that $\mathrm{X}$ and $\mathrm{Y}$ are different things-which is what $I$ will be asserting.

If it is a question of the definition of the term "iconic memory," then we must turn to the source of the term: chapter 2 of Neisser's Cognitive Psychology. The crucial passage in this locus classicus is as follows:

It seems certain, then, that the visual input can be briefly stored in some medium which is subject to very rapid decay. Before it has decayed, information can be read from this medium just as if the stimulus were still active. We can be equally certain that this storage is in some sense a "visual image." Sperling's subjects reported that the letters appeared to be visually present and legible at the time of the signal tone, even when the stimulus had actually been off for $150 \mathrm{msec}$. That is, although performance was based on "memory" from the experimenter's point of view, it was "perceptual" as far as the experience of the observers was concerned.

What should such a process be called? The subjects say they are looking at something, and it needs a name .... There seems no alternative but to introduce a new term for the transient visual memory in question. I will call it "the icon" or "iconic memory." (Neisser, 1967, pp. 18-20)

It will be seen that, unfortunately, Neisser has not been of assistance to us here, since he did not consider the possibility that visible persistence and iconic memory are different things; it certainly does not follow, simply because the stimulus continues to be visible after its physical offset, that this phenomenal persistence is the source of the information that is responsible for the partial report superiority. Neisser subsequently observed that "the icon is defined behaviorally and introspectively," which I take to mean that the partial-report superiority and introspections about phenomenal persistence were considered equally valid indices of iconic memory.

Although Neisser did not explicitly consider the possib. : need to distinguish informational persistence from phenomenal persistence, it is quite clear that it is informational persistence which was his primary concern: elsewhere in his book, he used terms such as "preliminary and transient storage mechanisms for sensory information" when discussing iconic memory. Furthermore, in two places in his book he does argue that iconic memory can exist even when it is not visible (which, of course, contradicts the views put forward in the long quotation I have just given from the book). On page 33, when discussing backward masking, he offers the following interpre- tation: "The subject reads letters not only from the stimulus while it is on but from the icon afterwards, despite the presence of the mask.... the display appears to be terminated by the onset of the mask"; and on page 35 he says: "It is hard to argue with a subject who says his 'image' terminated at a certain moment, but it seems apparent that the processing of iconic information can continue past this point." These two quotations make it clear that Neisser's view was that the backward mask terminates phenomenal persistence but not informational persistence; hence these two persistences are distinguishable entities; and, when a distinction is drawn between them, it is the informational persistence which is accorded the name "iconic memory." This is my justification for using "iconic memory" to refer to informational persistence, while "visible persistence" refers to phenomenal persistence.

We return, then, to the question of whether this terminological distinction corresponds to a realworld distinction; and we are now in a position to answer this question. The exhaustive (not to say exhausting) review of work on visible persistence presented earlier in this paper has provided ample evidence that visible persistence has the following two properties: its duration is inversely related to stimulus duration, and its duration is inversely related to stimulus luminance. If iconic memory is visible persistence, then iconic memory must possess these two properties too. Brief displays should generate longer iconic memories than longer lasting displays do. Low-luminance displays should generate longer iconic memories than high-luminance displays. If iconic memory does not display these two properties, then iconic memory is not visible persistence.

There are methodological pitfalls to be identified and avoided before considering whether iconic memory does have these two properties. Consider the following experiment. I wish to determine whether the duration of iconic memory is inversely related to stimulus luminance. Therefore, I present alphanumeric displays for a fixed, brief duration and use several luminance levels. The subject's task is to report as many of the items as he can. The rationale of the experiment is as follows: The longer iconic memory lasts, the better full report will be; if iconic memory duration is inversely related to stimulus luminance, then full report performance will also be inversely related to stimulus luminance.

Unfortunately, this will not do. As noted earlier, the limitation on full report is not imposed by the lifetime of iconic memory, but by a bottleneck of unknown origin higher up in the system, which prevents a subject from being able to report more than four or five items from a multi-item display. Increasing the lifetime of iconic memory has no effect on this bottleneck, so will have no effect on full report. 
Thus, if one failed to find any effect of stimulus luminance on full-report performance, this would not allow one to conclude that there was no effect of stimulus luminance on the duration of iconic memory.

Consider the following different approach: one uses 50-msec alphanumeric displays, and a backward mask at various stimulus-onset asynchronies (SOAs), requiring full report. The minimum SOA at which performance is no different from a no-masking control is determined. This SOA is taken as a measure of the duration of iconic memory, since it is inferred that the reason the mask has no effect at this SOA is that there is no longer anything to mask: iconic memory has terminated. The question of the relationship between stimulus luminance or duration and the duration of iconic memory is studied by varying stimulus luminance or duration and seeing what effect this has on the critical SOA.

This will not do either, for the same reason. A mask may be failing to have an effect at a certain SOA, not because iconic memory does not last as long as that SOA, but because enough information has been extracted from iconic memory to reach the bottleneck level: further information cannot be extracted from iconic memory, even though it is available. At this point, therefore, masking will not harm performance, even though iconic memory has not terminated. Therefore measuring the minimum SOA at which masking no longer impairs full report is not an appropriate way of measuring the duration of iconic memory.

What is needed is work using the partial-report technique. Even here, the bottleneck may be troublesome. If subjects engage in nonselective readout until they have decoded the partial report cue (as discussed earlier), it is conceivable that the bottleneck might be reached and so prevent utilization of the partial report cue. We know too little about the way iconic memory interacts with durable (i.e., posticonic) storage to be able to decide whether this is a real possibility. It will be neglected here.

\section{Effect of Stimulus Luminance on Iconic Memory}

This was first studied, using the partial report technique, by Keele and Chase (1967). They used displays consisting of 10 alphanumeric items, arranged in a 2.5-deg-diam circle around a fixation point. Display duration was $100 \mathrm{msec}$; the letters were black on a white ground, and luminance levels of 3.7 , 16 , and $70 \mathrm{fL}$ were used. The partial report cue was an arrow pointing at one of the 10 display positions. Its duration was $150 \mathrm{msec}$. Except when the display or the cue was present, the exposure field was dark. Cue delays from 0 msec (i.e., at display offset) to $5,000 \mathrm{msec}$ were used.

Unfortunately, it is difficult to decide what these data tell us about the relationship between iconic memory and visible persistence, for two reasons. The first is that two of the three stimulus luminances used by Keele and Chase were much higher than those used in studies of visible persistence and would have produced retinal illumination in excesss of $1,000 \mathrm{Td}$ if pupil diameter were $4.8 \mathrm{~mm}$ or more (a diameter which, according to the formula of LeGrand, given earlier, would be produced by an adapting field of a little less than $1 \mathrm{fL}$; the subjects in Keele and Chase's experiment were briefly dark adapted and viewed a dark field except when display or cue was briefly presented). Since displays having such high levels of retinal illumination may not yield visible persistence as defined earlier, but instead a form of afterimage (the "weak afterimage" of Sakitt, 1976b), comparison of the results of Keele and Chase (1967) with results of experiments on visible persistence is probably not relevant to the question of whether iconic memory is visible persistence.

A second problem is that, if we wish to decide how luminance affected rate of decay of iconic memory, the decision is complicated by the fact that performance was already affected by luminance at zero delay of the cue in Keele and Chase's data. Since, at long cue delays, performance asymptotes at a level which must be independent of luminance (because of the bottleneck problem described earlier), there must be an interaction between luminance and cue delay: but this could be because luminance affects initial registration in iconic memory, not rate of decay. The only satisfactory way around this problem is to seek conditions in which luminance demonstrably does not affect initial registration in iconic memory; this has been done by Adelson and Jonides (Note 3), whose work is described below. A less satisfactory alternative is to assume that iconic decay rate is (1) exponential, and (2) unaffected by level of initial registration; given these assumptions, one could fit exponential functions and measure decay rate by exponential half-life. In the absence of either of these approaches, Keele and Chase's data do not provide adequate information concerning whether iconic memory and visible persistence are influenced in the same way by variations in stimulus luminance.

Eriksen and Rohrbaugh (1970) used 2.0-deg-diam circular displays containing 12 letters. The letter to be reported was indicated by a bar pointing to it or by a black ring encircling the display position it had occupied. Display durations ranged across subjects from 35 to $45 \mathrm{msec}$. Display luminance was .7 or $7.0 \mathrm{~mL}$. The luminances of the preexposure field, of the field intervening between display and letter indicator, and of the letter-indicator field were always equal to the luminance of the display. Indicator delays from 0 to $300 \mathrm{msec}$ were used.

With the bar marker, performance declined monotonically with indicator delay, as expected. Performance was better with the high-luminance display. 
The higher luminance advantage did not interact significantly with indicator delay; but it should be noted that the higher luminance display also had a higher luminance postexposure field to contend with. At any rate, it was clear that there was no evidence at all to suggest that iconic memory lasts longer when display luminance is low than when it is high: display luminance had no effect on iconic memory duration. It should be noted that the retinal illumination produced by a $7.0-\mathrm{mL}$ stimulus and $7.0-\mathrm{mL}$ adapting field is well below $1,000 \mathrm{Td}$.

The ring condition is not relevant here since it involves masking rather than purely iconic memory decay. (Averbach \& Coriell, 1961).

Scharf and Lefton (1970) used displays consisting of three rows of four letters, with an auditory post exposure cue requesting the report of one row. A white field was present between display and cue. Two display luminances, .12 and $12 \mathrm{fL}$, and two cue delays, 0 and $500 \mathrm{msec}$, were used. The size of the cue delay effect was the same for the two display luminances. Again, then, clear evidence was obtained for the absence of any inverse relationship between stimulus luminance and duration of iconic memory. Here, again, duration of iconic memory is simply unrelated to display luminance, and in conditions where retinal illumination was below $1,000 \mathrm{Td}$.

Adelson and Jonides (Note 3) used 8-letter displays, the letters being positioned around the circumference of an imaginary 1.68-deg-diam circle. Display duration was $50 \mathrm{msec}$. One of the letters was cued for report by a 50 -msec bar marker. The interval between display offset and bar marker onset varied from 0 to $950 \mathrm{msec}$, and in addition there was a precue condition: the bar marker was presented $100 \mathrm{msec}$ before the display. As will be seen, this is an important aspect of their experiment. An adapting field of $9 \mathrm{~mL}$ was used. There were four levels of display luminance: $8.7,17.5,35$, and $70 \mathrm{~mL}$.

The precue condition yielded almost $100 \%$ accuracy at the three highest display luminances. Thus one could be sure that, in this range, display luminance was not influencing registration in iconic memory, so that any luminance effects with delayed cues could be attributed to influences on decay of iconic memory. However, there were no such effects: The functions relating performance to cue delay were identical at the three different display luminances. Even with the lowest luminance display, where performance with a precue was not perfect, partial report performance decayed at the same rate.

In a second experiment, using the same partial report method, Adelson and Jonides orthogonally varied display luminance ( 25 and $75 \mathrm{~mL}$ ) and adapting field luminance (10 and $50 \mathrm{~mL})$. These four conditions yielded indistinguishable functions when partial-report performance was plotted against cue delay.
As Adelson and Jonides note, it is possible to devise conditions under which increases in luminance produce increases in the duration of iconic memory: one uses extremely intense stimulation, dark-adapted subjects, and large stimuli. This was done by Sakitt (1976a, 1976b); under these conditions, an afterimage is generated. This is discussed further below; it is sufficient here to point out that these conditions are abnormal in relation to conventional tachistoscopic studies of iconic memory, which have used luminance levels too low to generate afterimages, relatively small stimuli, and (usually) light-adapted subjects.

It should also be noted that Averbach and Sperling (1961) and Sperling (1960, Table 20) found that partial report performance was much worse with a light postexposure field than with a dark postexposure field. Thus, in the limit, adapting field luminance does influence partial report performance. This does not affect the conclusion that, within the luminance range characteristic of tachistoscopic experiments, display luminance has no effect on iconic memory duration, provided that the luminance is high enough to ensure full legibility of the display initially.

The outcome of these studies of the effect of display luminance on iconic memory duration is clear. No study provided any evidence that there might be an inverse relationship between these two variables. Numerous studies of visible persistence have indicated that there is an inverse relationship between display luminance and the duration of visible persistence. We must conclude that iconic memory and visible persistence behave quite differently when display luminance is varied.

\section{Effect of Stimulus Duration on Iconic Memory}

Sperling (1960, Figure 4) found that increases in exposure duration over the range 15 to $500 \mathrm{msec}$ did not affect full report of tachistoscopic displays. Mackworth (1963, Figure 1) found that full report did improve considerably as exposure duration increased from 27 to $125 \mathrm{msec}$, but, since display luminance $(3.9 \mathrm{fL})$ was much lower than adapting field luminance ( $32 \mathrm{fL}$ ), this result is not surprising. With a dark adapting field, exposure duration had a significant but extremely small effect. These results, however, since obtained with full report, do not provide clear evidence concerning how exposure duration affects the duration of iconic memory.

Data for partial report are given by Sperling (1960, p. 14). With a dark postexposure field and zero cue delay, average partial report performance was $\mathbf{8 . 0}$ letters for a 15-msec display and 8.2 letters for a 50 -msec display. With a cue delay of $150 \mathrm{msec}$, these values were 6.5 letters for the 15 -msec display and 7.2 letters for the 50 -msec display. There is thus a very small effect of exposure duration. Sperling did not 
report whether it was statistically significant; in any case, it is quite clear that increasing display duration does not decrease iconic memory duration; if it has any effect at all, the effect is to increase iconic memory duration.

The effect of display duration on partial-report performance has also been studied by Di Lollo (1978). In his Experiment 2, a row of 7 letters was followed immediately by a frame around one of the letter positions, specifying which letter to report. Figure 4 of Di Lollo (1978) showed that performance was identical with display durations of 100 and $200 \mathrm{msec}$. Since a postexposure cue at display offset is not actually applied to the contents of iconic memory until up to $270 \mathrm{msec}$ after display offset (Averbach \& Coriell, 1961), because of cue-decoding time, we would expect an effect of exposure duration in Di Lollo's experiment if the decay rate of iconic memory were affected by exposure duration. In particular, if long displays produced shorter iconic memories, partial report performance should be worse with the 200 -msec display than with the 100-msec display. This did not happen. Thus, both Di Lollo (1978) and Sperling (1960) have demonstrated that there is not an inverse relationship between display duration and iconic memory duration. Numerous studies of visible persistence have indicated that there is an inverse relationship between display duration and the duration of visible persistence. We must conclude that iconic memory and visible persistence behave quite differently when display duration is varied.

\section{Is Iconic Memory Visible Persistence?}

The duration of visible persistence is highly sensitive to physical parameters of the display: the luminance and the duration of the display. Various studies using various techniques have shown that increases in display luminance or duration result in decreases in the duration of visible persistence. Iconic memory behaves quite differently. Provided display luminance and duration are adequate for legibility, and provided that luminance is not so high that afterimages are generated, the duration of iconic memory is simply not affected by variations in the luminance or duration of the display. I submit that this is conclusive evidence that iconic memory is not visible persistence.

\section{THE NEURAL BASES OF ICONIC MEMORY AND VISIBLE PERSISTENCE}

Earlier in this paper, it was suggested that we need to distinguish between three forms of visual persistence: informational persistence ("iconic memory"), visible persistence, and neural persistence. Subsequently, I have argued that the distinction between iconic memory and visible persistence is not merely terminological: they are actually different psychological processes. Given this, one must separate a discussion of the neural basis of iconic memory from a discussion of the neural basis of visible persistence. In this section, I discuss neural persistence from these two points of view.

It is necessary to begin by saying something about afterimages. According to Julesz (1971), iconic memory is "merely an afterimage"; according to Neisser (1967, p. 141), "iconic memory may not be related to the afterimage at all"; according to Sperling $(1960$, p. 22$)$, iconic memory is not an afterimage.

\section{Afterimages}

It has been proposed by Sakitt (1976b) that there are three qualitatively distinct categories of afterimage. Her terms for these are strong afterimage, weak afterimage, and ultraweak afterimage. A major difference between these three categories is the stimulus luminance required to evoke the afterimage: roughly speaking, the strong afterimage requires intense stimulation, the weak afterimage is generated by stimuli of moderate intensity, and the ultraweak afterimage is generated by stimuli at or somewhat above threshold intensity.

Strong afterimages are produced by lights that are intense enough to bleach a substantial fraction (more than $1 \%$ ) of the rod photochemical, rhodopsin. The properties of these afterimages, Sakitt argues, are well described by the photochemical kinetics hypothesis advanced by Alpern and Barr (1962). However, not all afterimages can be dealt with by this hypothesis: "The photochemical kinetic theory, which explains strong afterimages very well, predicts no strong afterimage for stimuli below 3.8 log scotopic trolands" (Sakitt, 1976b, p. 135); yet afterimages were produced by considerably less intense stimuli in Sakitt's experiments. Furthermore, afterimages were produced by stimuli which bleached considerably less than $1 \%$ of available rhodopsin: Sakitt calculated that bleaching of only $5.8 \times 10^{-5}$ of rhodopsin produced an afterimage. The photochemical theory predicts that such a small amount of bleaching would not produce an afterimage. Sakitt concluded that, although the strong afterimage exists and is well described by the Alpern-Barr hypothesis, it is not the only kind of afterimage.

The weak afterimage is produced by lights which bleach substantially less than $1 \%$ of rhodopsin. This afterimage lasts from $250 \mathrm{msec}$ or so up to several seconds. Its duration depends upon light intensity: the more intense the light, the longer the weak afterimage lasts. The weak afterimage requires a minimum intensity of light-about $5 \mathrm{log}$ units above rod threshold-for its generation.

Stimuli that are too weak even to evoke the weak afterimage produce the ultraweak afterimage: its 
duration is about $160 \mathrm{msec}$, and this duration, Sakitt claimed, is unaffected by variation in stimulus intensity.

Sakitt argued that both the weak and the ultraweak afterimages correspond to visual processes studied previously under other names. The ultraweak afterimage is that which has previously been called "visual persistence": "There seems to be a minimum subjective duration of any visual stimulus, no matter how weak the intensity of the light. This implies visual persistence'' (Sakitt, 1976b, p. 129). The weak afterimage, Sakitt argues, is what previously has been called "iconic memory."

The strong afterimage is not of interest here, since it requires stimulus intensities far above those that have been used in studies of visible persistence and of iconic memory. Sakitt's identification of the weak afterimage with iconic memory, and the ultraweak afterimage with visual persistence, is, however, clearly something which requires discussion.

Central to the distinction between these two types of afterimage is the concept of rod saturation. As stimulus intensity is increased, the rod response incre. $s$, up to a critical intensity: further increases in stimulus intensity do not produce further increases in rod response. At this point, the rod is said to be saturated: about 1,000 scotopic trolands is the level of retinal illumination at which rod saturation occurs. Sakitt (1976b) proposed a model for the weak and ultraweak afterimages, based upon the phenomenon of rod saturation. Subsaturating intensities generated the ultraweak afterimage; intensities at or greater than that required for rod saturation generated the weak afterimage.

The model will not be discussed in detail here, since it appears to be inconsistent with the data on weak and ultraweak afterimages. For example, the model predicts that the duration of the ultraweak afterimage is independent of stimulus intensity. This appeared to be so in Sakitt's data (Sakitt, 1976b), but she acknowledged that her measurement technique was not sensitive enough to detect small changes in afterimage duration. Elsewhere (Sakitt, 1976a), she acknowledged that increases in stimulus intensity produce decreases in the duration of the ultraweak afterimage or visual persistence. As the earlier review of this work has indicated, there is an inverse relationship between stimulus intensity and the duration of visual persistence, and the existence of this relationship refutes Sakitt's model of the ultraweak afterimage. Her model of the weak afterimage predicts that the relationship of stimulus intensity to afterimage duration should be a series of steps, not a smoothly increasing function; yet she observed a smoothly increasing function.

Although the detailed model proposed by Sakitt (1976b) is rejected here, the distinction between the ultraweak afterimage (visible persistence) and the weak afterimage is strongly supported by her data. Furthermore, since the retinal illumination level below which the ultraweak afterimage occurs and above which the weak afterimage occurs is about $1,000 \mathrm{Td}$, which is the minimum illumination required for rod saturation, Sakitt's view that the weak afterimage is produced by rod saturation is a plausible one.

However, her claim that iconic memory is the weak afterimage cannot be correct. This claim implies at least the following two statements: (1) that stimuli which are not intense enough to saturate the rods will not produce iconic memory, because they cannot produce weak afterimages; and (2) that iconic memory cannot exist if a postexposure field is used which is intense enough to saturate the rods. Both of these implications of Sakitt's view can be refuted by reference to data from experiments on iconic memory.

First, there are many studies of iconic memory in which stimuli too weak to saturate the rods and hence generate a weak afterimage still produce iconic memory. For example, Adelson (1978) obtained a clear partial report superiority which declined with cue delay, using letters producing a retinal illumination of $1.5 \mathrm{log}$ Td on a $2.2-\log$-Td background. Here neither letters nor background could have saturated the rods. Eriksen and Rohrbaugh (1970) obtained classical iconic memory effects with a luminance of $.7 \mathrm{~mL}$; even at maximum pupil dilation, this corresponds to a retinal illumination of about $112 \mathrm{Td}$, an order of magnitude too small to saturate the rods. There are numerous other such examples. Sakitt (1976a, p. 265) has argued that most tachistoscopes operate in a luminance range which includes luminances that will saturate the rods, but, even if this is so, many investigations of iconic memory do not employ the maximum luminance of which the investigator's tachistoscope is capable; and, indeed, some tachistoscopes cannot produce stimuli intense enough to saturate the rods [such as, for example, the tachistoscope used by Sakitt and Appelman (1978)-see p. 563 of their paper].

Secondly, in the classic study of iconic memory by Averbach and Coriell, an adapting field of luminance $70 \mathrm{fL}$ was maintained constantly throughout the experiment. This, accordingly to LeGrand's formula, should produce a pupil diameter of $2.78 \mathrm{~mm}$ and hence a retinal illumination of about $1,450 \mathrm{Td}-$ well above what is required for rod saturation. Averbach and Coriell's stimuli were black letters (luminance $1 \mathrm{fL}$ ) on the 70 -fL background. If iconic memory requires rod saturation-i.e., if the rods stimulated by the background were saturated while those stimulated by the letters were not, and this gave rise to an iconic memory after display offset-the postex- 
posure field, since it saturates all the rods (including those which had been stimulated by the display letters), should eliminate iconic memory. Yet, a rapid decay of performance as bar-marker delay increased was obtained by Averbach and Coriell.

Thus, it is not necessary to saturate any rods to produce iconic memory; and if one saturates all rods, one does not wipe out iconic memory.

I have argued earlier that iconic memory is not visible persistence, that is, is not, in Sakitt's terminology, the ultraweak afterimage. Here I argue that it is not the weak afterimage either.

This is not to say, of course, that the distinction between ultraweak and weak afterimages is to be rejected. Indeed, this distinction is evident in earlier work: In a passage cited previously in this paper, Allport (1970, p. 687) observed that, in his experiments with stroboscopic illumination of a rotating radius, observers sometimes reported afterimages, which they distinguished from the visible persistences of the rotating radius. In addition, Allport pointed out that, if the intensity of the stroboscopic flash was reduced, the duration of visible persistence was increased while, at the same time, the duration of the afterimages was decreased. Evidently, both the weak and ultraweak afterimage were present.

Nor would I wish to claim that partial report superiority cannot be mediated by afterimages. Afterimages exist and they decay away over time. If the time required to report all the items in an afterimage exceeds the lifetime of the afterimage, while the time required to report those items specified by a partial report cue is less than the afterimage lifetime, then afterimages must be able to produce a superiority of partial report over full report. That is why Sakitt (1976a) was able to produce a partial report superiority in a rod monochromat using a display whose letters were initially invisible (invisible because even the dimmer background surrounding the letters was intense enough to saturate the rods). The weak afterimage was generated, and this produced a partial report superiority.

Afterimages are thus sufficient to generate a partial report superiority. When discussing whether they are necessary, we must use the term "afterimage" with precision. Two types of afterimage which are relevant are the ultraweak and the weak afterimage. Iconic memory can occur with stimuli too weak to produce the weak afterimage, and in these circumstances the properties of iconic memory do not correspond to the properties of the ultraweak afterimage (otherwise known as visible persistence). It follows that circumstances exist in which iconic memory effects (such as partial report superiority) can be observed without their being produced by afterimages. Hence, afterimages are not necessary for partialreport superiority to occur.

\section{Rods and Cones}

The model proposed by Sakitt (1976b) asserted that both the ultraweak afterimage, or visible persistence, and the weak afterimage, or iconic memory, were properties of the rod system. Even if the model is wrong, this assertion may be correct; certainly Sakitt has reiterated it in subsequent publications (Sakitt \& Long, 1978, 1979). It is clear enough that the weak afterimage is a rod phenomenon, since it exhibits the spectral sensitivity of the rods, emerges as soon as retinal illumination is sufficient to saturate the rods, and is present in a rod monochromat (Sakitt, 1976a); but I have argued that iconic memory is not the weak afterimage, so these observations by Sakitt are not relevant to the issue of the involvement of rods in iconic memory.

Adelson (1978) has studied the relative contributions of rods and cones to both visible persistence (as defined by the synchrony-judgment technique) and iconic memory (as defined by the partial-report technique). With dark-adapted subjects, iconic memory was studied when stimuli were visible only to cones (because letters were scotopically matched to the background), when stimuli were visible to the rods and cones because of scotopic mismatch, and when stimuli were visible only to rods (a color-blind subject was used here). In all three situations, a partial report superiority which declined with cue delay was observed. Thus, neither rods nor cones are necessary for iconic memory; rods are sufficient, and so are cones. Results consistent with this conclusion have also been reported by Banks and Barber (1977).

When the color-blind subject was light-adapted, a decaying partial report superiority was observed when stimuli were visible only to the cones; when stimuli were visible only to the rods, no letters could be reported.

Visible persistence was measured, with dark-adapted subjects, by adjusting an auditory stimulus until it coincided in time with the apparent offset of the 50-msec alphanumeric visual display. Persistence durations of up to $900 \mathrm{msec}$ were obtained. Such durations are very much greater than any obtained in previous work; the reason for this remains unknown. Stimuli visible only to rods persisted longer than stimuli visible only to cones; stimuli visible to rods and cones persisted longer than stimuli visible only to cones.

Thus, the cones do produce visible persistence, but the rods produce longer-lasting visible persistences than the cones. ${ }^{8}$ Since the duration of iconic memory did not depend on receptor type in this way, Adelson's experiments provide further evidence that iconic memory is not the same thing as visible persistence, and, indeed, he pointed out that "the phenomenal persistence of a stimulus ... does not 
necessarily depend on the same processes as visual storage measured by partial-report advantage."

These studies of rod and cone contributions to visible persistence and iconic memory yield conclusions similar to those reached when one considers studies of the effects of stimulus duration and luminance on visible persistence and iconic memory. Visible persistence is strongly affected by variations in stimulus parameters, and by the contributions of rods vs. cones. Iconic memory is indifferent to these stimulus parameters, and also to the type of receptor scited by the stimulus: as long as the stimulus is sufficiently legible, its duration or luminance, and the type of receptor involved, is not relevant as far as iconic memory is concerned. This suggests that visible persistence is intimately tied to processes going on in the visual pathways from retina to visual cortex, while iconic memory is a property of some much higher stage in the informationprocessing sequence. The next topic to be discussed is the stage or stages in the visual pathway that the neural events responsible for visible persistence occur.

\section{Persistence in the Photoreceptors}

As the work by Adelson (1978), discussed above, shows, rods and cones behave differently in experiments on visible persistence, and hence there is reason to believe that photoreceptor activity constitutes one neural locus for visible persistence. The model for visible persistence and the weak afterimage proposed by Sakitt (1976b) specifically attributes both effects to activities in the rods; but, as noted earlier, this model cannot be correct for visible persistence (because it is refuted by the inverse relationship between luminance and persistence duration) and is dubious for the weak afterimage (because it predicts that the luminance-duration function will be a series of step functions, when in fact it is monotonic). However, even if this model is incorrect in detail, it is clearly worth considering data from electrophysiological studies of photoreceptor activity, in order to see whether any phenomena emerge there which correspond to what has been observed in psychophysical studies of visible persistence.

Recordings by Whitten and Brown (1973a, 1973b, 1973c) of the late receptor potential (RP) of rods and cones in the macaque monkey (whose retina is very similar to that of the human), as well as in other species (cat, rat, and mudpuppy) by Fain and Dowling (1973), Penn and Hagins (1972), and Steinberg (1969), have shown that, after stimulus offset, both the rods and the cones continue to output signals for some time. The cone signal, however, decays away rapidly, whereas the rod signal decays with a much longer time constant (see, for example, Whitten \& Brown, 1973a, Figures 7, 9, and 14a). This finding, of course, is paralleled by the psychophysical results of Adelson (1978), discussed earlier. Thus, persistence exists at the photoreceptor level in the visual system, and consequently, if we are considering the question of what the neural bases of visible persistence might be, we must begin at the beginning, the photoreceptor level. It might even be argued that, since a persistence arising at any one stage of the visual system must be passed on through subsequent stages, photoreceptor persistence must have effects throughout the visual system, and so have perceptual consequences; but this argument is too strong, since it is, in principle, possible for persistence introduced by any stage in the visual system to be removed by some later stage, through inhibitory mechanisms. So one cannot take it for granted that the photoreceptor persistence has perceptual consequences; but this is obviously a possibility which deserves examination. At low (scotopic) intensities, only the slowly decaying rod RP is elicited. As intensity increases, there is a short intensity range (mesopic) in which a mixed rod/cone $\mathrm{RP}$, containing both a fast-decaying and a slowdecaying component, appears. At higher intensity values (photopic levels), where the cone RP is well above threshold, rod-saturation level is reached, and one would therefore expect a maximal rod RP; but, instead, there is no rod RP at all, only the fast-decaying cone RP. Whitten and Brown (1973b) claimed that this effect occurs because cones inhibit rods. This effect was subsequently demonstrated in the human with psychophysical techniques by Makous and Boothe (1974) and Stabell and Stabell (1976). The inhibition appears to be carried out by horizontal cells, which are known to link cones with rods in the macaque retina.

It is not clear how this suppression of rods by cones is related to the phenomenon of rod saturation. Certainly cone inhibition is not responsible for rod saturation, since rod monochromats exhibit rod saturation (e.g., Sakitt, 1976a, 1976b).

In the macaque or human retina, rods and cones feed onto bipolar cells, any bipolar cell receiving only rod input or only cone input; bipolar cells then feed onto ganglion cells. A ganglion cell receives input from both rod bipolars and cone bipolars, so activity in a ganglion cell reflects both cone activity and rod activity. In addition to these "vertical" pathways, there are "horizontal" pathways between rod and cone-the horizontal cells, presumed to mediate cone-rod inhibition.

The existence of this inhibition means that, at scotopic intensity levels, all ganglion cell activity is produced by rod input; at a mesopic range of intensities, the ganglion cell is receiving both rod and cone inputs; and at all higher intensities, rod signals are prevented by cone inhibition from reaching the ganglion cell, so that, except for very peripheral 
parts of the retina where there are no cones, activity in the ganglion cell and in all subsequent stages of the visual system will depend solely on the cones' responses to the visual stimulus.

When anoxia or anaesthesia was used to free the rods from cone inhibition, it was found that the latency of the rod RP decreased linearly as log stimulus luminance increased, up to the luminance level which saturated the rods. Up to this level, however, rise and decay times of the rod RP (and hence total duration of the rod response) were unrelated to luminance. Above the saturating level, decay time was influenced by luminance, higher luminances producing slower decay. However, under normal conditions, cone inhibition is sufficient to suppress the rod response entirely at luminances somewhat below rod-saturation level.

A similar pattern occurs with the cones. As luminance increases from cone threshold up into the high photopic range, rise and decay times of the cone response do not change, nor is latency of the response (which is small-about $20 \mathrm{msec}$ ) affected. Only at very high luminances does luminance begin to affect decay time; here an increase in luminance produced a slower cone decay.

Therefore, as luminance is varied from near the rod absolute threshold up into the high photopic range, photoreceptor persistence of input to ganglion cells will behave as follows. In the scotopic range, where only the rods are functioning, and so rods are free from cone inhibition, the duration of input to the ganglion cells will be independent of luminance (though the latency of the onset and the offset of this input will decrease, equally, as stimulus luminance increases), and hence the duration of visible persistence, to the extent to which this is determined by photoreceptor persistence, will be independent of stimulus luminance, when scotopic luminances are used.

In the mesopic range, cones will be responding, and hence inhibition of the rods will be occurring, but the inhibition will not be strong enough to produce complete suppression of the rod response. Instead, the inhibition merely reduces the amplitude and persistence of the rod response. Despite this partial inhibition, the rod response still persists for longer than the cone response. However, as luminance increases through the mesopic range, the cone response, and hence cone-generated inhibition, becomes stronger and stronger, and so rod persistence becomes briefer and briefer, until one reaches the luminance level at which the rod signal is completely suppressed and only cones signal to the ganglion cells. Within the mesopic range, then, the duration of photoreceptor input to ganglion cells will become shorter and shorter as luminance is increased and rod persistence shortened, until this duration asymptotes to the duration of the cone response. Hence, the duration of visible persistence, to the extent to which it is determined by photoreceptor persistence, will be inversely related to stimulus luminance, when mesopic luminance levels are used.

At moderate to high photopic levels, latencies and decay times of cones are uninfluenced by stimulus luminance. Thus, further increases in stimulus luminance, beyond the luminance necessary for complete rod suppression, will have no effect on photoreceptor persistence duration, until the very high photopic levels which prolong cone decay time are reached.

In sum, then, at low and at moderately high stimulus luminances, the duration of the persistence of photoreceptor input to ganglion cells is independent of luminance; but there exists a luminance range in which photoreceptor persistence duration is inversely related to luminance. Since this is true for visible persistence too, it is natural to pursue the possibility that a (or the) neural basis of visible persistence is photoreceptor persistence; it is even more natural to pursue the possibility that the inverse relationship between stimulus luminance and persistence duration is due to inhibition of rods by cones, even if other properties of visible persistence must be assigned other loci within the visual system. Arguments based on Whitten and Brown's work require that the range over which luminance has an inverse effect on persistence duration has, at its lower bound, absolute thresholds for cones (since, below this value, cone inhibition cannot occur) and, as its upper bound, rod saturation level (since, above this value, rod responses are completely suppressed). In practice, the luminance range would be somewhat smaller, because effective inhibition might require a cone response somewhat stronger than the threshold response, and because rod persistence would be shorter than cone persistence at luminancs near, though not at, the level producing rod saturation, and this is all that is required for a stimulusluminance effect no longer to be evident.

As noted earlier, virtually all of the work on visible persistence has used stimuli producing retinal illuminations of less than $1,000 \mathrm{Td}$, that is, less than that required for rod saturation. It is also true that all of this work has used stimuli well above cone threshold. Consequently, the numerous studies which have observed an inverse relationship between stimulus luminance and the duration of visible persistence have used stimuli with liminances confined within a range bounded below by cone threshold and above by rod saturation level. Thus, all of these data are consistent with the argument that the inverse luminance effect can be attributed to cone inhibition of rods; but a stringent test of this argument requires 
the use of stimuli below cone threshold. If, with these stimuli, the inverse luminance effect no longer occurs, this would be strong support for the argument.

The other major property of visible persistence-the finding that its duration is inversely related to stimulus duration-could conceivably be a photoreceptor effect too. To propose this, one would need to postulate that the cone inhibition of rods was more intense for longer lasting stimuli, as if the cone inhibitory effect accumlated during the presence of the stimulus. This could be investigated in experiments like those of Whitten and Brown if stimulus duration were varied systematically, something which they did not try. However, it seems unlikely that this approach to explanation of the duration effect will prove fruitful. If the effect of increasing luminance and of increasing duration is to amplify the inhibitory influence of cones upon rods, one would expect that when both stimulus variables are manipulated, their effects upon persistence duration would be interactive, not additive. For example, the maximum luminance at which a luminance effect is still evident should be less for long stimuli (which themselves would contribute much inhibition) than for brief stimuli (which would contribute little). However, in the results of Bowen et al. (1974), stimulus luminance and stimulus duration have additive effects upon the duration of visible persistence; over durations from 5 to $350 \mathrm{msec}$, the luminance effect is the same size, and this is also true over durations from 25 to $1,000 \mathrm{msec}$. Thus, if the photoreceptors are responsible for the luminance effect, one is led to look for a different locus for the duration effect. This is one reason for rejecting the view that the sole neural basis for visible persistence is photoreceptor persistence. Another reason for rejecting this view is, of course, the fact that the stereoscopic visible persistence studied by Engel (1970) must have a cortical locus; similarly, it is not evident how the dichoptic results obtained by Briggs and Kinsbourne (1972) could have occurred if persistence were purely retinal.

Nevertheless, the photoreceptor persistence demonstrated by Whitten and Brown must result in perceptible persistence unless higher stages in the visual system intervene to remove the photoreceptor effect, and there is no evidence that this occurs. Furthermore, the view that one neural basis for visible persistence is photoreceptor persistence does offer a bonus, namely, an attractive explanation of the inverse luminance effect. Thus, I will take the view that photoreceptor persistence is responsible for some, but certainly not all, visible persistence effects.

An explanation of visible persistence solely in terms of photoreceptor persistence is ruled out not only by the evidence adduced above, but also by the finding that the duration of the visible persistence of a square-wave grating depends upon the grating's spatial frequency, high frequencies yielding longer persistences (Meyer \& Maguire, 1977). There is no reason why this should happen if persistence occurred solely at the photoreceptor level. On the other hand, it is precisely what would be expected if, as well as there being a form of neural persistence intrinsic to the photoreceptors, cells of the pathway from ganglion cell to lateral geniculate to visual cortex also possessed their own intrinsic persistences. Evidence has been rapidly accumulating over the past decade that at least one class of cell in this pathway does possess such persistence-the "sustained" cell.

\section{Sustained and Transient Cells}

When recordings are made from units in the visual system at the level of ganglion cell, lateral geniculate nucleus, or visual cortex, two different types of cell are encountered: transient cells and sustained cells. When a stimulus is switched on, the transient cells respond to its onset with a brief burst of activity; somewhat later, the sustained cells begin to respond. If the stimulus is kept on, activity in transient cells soon ceases, but the sustained cells continue to respond. Then, at the offset of the stimulus, transient cells again briefly respond, whereas the activity of the sustained cells merely begins to diminish and progressively returns to its prestimulus level. The two types of cell differ in numerous other ways. Not only do transient cells have shorter response latencies; they also have faster conduction velocities and larger receptive fields than sustained cells. Perhaps because of the differences in receptive-field sizes, transient cells are most responsive to low spatial frequencies, while sustained cells are most responsive to high spatial frequencies. In response to a 2-msec flash of light, the transient-cell response not only has a shorter latency, but the whole response may last for only about half as long as the sustainedcell response (Cleland, Levick, \& Sanderson, 1973). The latter authors also showed that the changeover from rod function to cone function as background illumination increases (the Purkinje shift) is evident for both types of cell, which indicates that both types of cell receive input from both types of photoreceptor. Although it has sometimes been suggested that transient cells are less common in the fovea or area centralis, a detailed histological study by Wässle, Levick, and Cleland (1975) showed, at least for the cat, that the percentage of so-called "alpha" ganglion cells, which are almost certainly transient cells, is the same in the area centralis as it is in the other retinal regions of various eccentricities.

These electrophysiological findings are very closely paralleled by psychophysical results obtained with human observers. For example, Breitmeyer (1975) showed that simple RT to the onset of a sinusoidal 
grating increased with spatial frequency, and Vassilev and Mitov (1976) obtained a similar increase for choice RT to square-wave gratings. This is evidence for the existence of channels which prefer low spatial frequencies and which have shorter latencies and/or faster conduciton times than channels which prefer high spatial frequencies. Parker and Salzen (1977) obtained further evidence of this kind by showing that the latency of early components of the visual evoked response increased as the spatial frequency of the evoking stimulus, a sinusoidal grating, increased.

Breitmeyer and Julesz (1975) compared contrast sensitivities for gratings which had abrupt temporal onsets to contrast sensitivities for gratings which had slow temporal onsets. For spatial frequencies of .5 cycles/deg to about 3 to 5 cycles/deg, sensitivity was higher for abrupt-onset gratings than for slowonset gratings. For spatial frequencies in the range 8 to 15 cycles/deg, steepness of temporal onset did not affect sensitivity. This is consistent with the view that an important contribution to the detection of low-frequency gratings is made by units which are particularly responsive to abrupt onset (i.e., transient cells), whereas these units do not contribute to the detection of high-frequency gratings.

Since sustained channels continue to respond throughout the period for which a stimulus is present, one might consider activity in these channels to subserve the perception of the stimulus as being continuously present; if so, the fact that such channels continue to respond for some time after physical offset of the stimulus would mean that the stimulus would continue to be seen for some time after its offset, that is, visible persistence would occur. An idea obviously worth exploring, then, is that one neural basis for visible persistence is continuing activity in sustained channels after stimulus offset; and so it is worth considering the extent to which the properties of the sustained and transient systems, as they are currently understood, can explain the data of the various studies of visible persistence reviewed earlier in this chapter.

\section{Sustained Channels and Visible Persistence}

The finding that sustained channels are selectively sensitive to high spatial frequencies seems likely to be related to the finding that high spatial frequencies have longer lasting visible persistences than low spatial frequencies. However, underlying this suggestion would seem to be the assumption that channels that are strongly stimulated will yield long persistence durations; and we have already seen that increases in stimulus luminance reduce persistence duration. To deal with this problem, I will rely on an elaboration of the proposal of Breitmeyer and Ganz (1977) to the effect that transient channels inhibit sustained channels. They proposed that this happened when a sudden new visual stimulus was presented (as when an eye movement occurred, or in backwardmasking experiments); an additional possibility is that inhibition of sustained by transient cells occurs as a consequence of the response of transient cells to stimulus offset. This inhibition would have the effect of reducing or terminating the visible persistence produced by activity which continues in sustained channels after stimulus offset, in just the same way that inhibition from cones could reduce or terminate the visible persistence produced by rods.

If the strength of this inhibitory effect were proportional to stimulus intensity, then the inhibition would be faster or stronger for intense stimuli than for weak stimuli. If so, visible persistence would be more effectively curtailed when the stimulus was intense than when it was weak. Thus, the plausible suggestion that there is a direct relationship between stimulus intensity and the strength or latency of the inhibition exerted by transient channels upon sustained channels leads to the prediction that the duration of visible persistence will be inversely related to stimulus intensity.

It is perhaps worth mentioning here that there is obviously a difference between experiments in which subjects are asked whether a form appears continuously present (phenomenal-continuity experiments) and those in which subjects are asked to detect flicker. Flicker experiments produce much shorter estimates of "persistence." However, as noted by Meyer and Maguire (1977), it is possible to see flicker even while a form appears continuously present. This is presumably because flicker detection depends on transient channels, while phenomenal continuity depends upon sustained channels. The finding that "persistence times," as estimated in flicker experiments, decrease as luminance increases (i.e., critical fusion frequency increases as luminance increases) is evidence that increasing luminance does reduce the latency of response of transient channels.

Nevertheless, there are some unattractive features of this proposed explanation of the luminance effect. First, the effect seems too large. Allport (1970) found that an increase of $1.74 \mathrm{log}$ units of stimulus luminance reduced persistence duration from about $100 \mathrm{msec}$ to about $50 \mathrm{msec}$, and Efron (1970c) found that an increase in luminance of about the same amount reduced persistence duration from about $140 \mathrm{msec}$ to less than $70 \mathrm{msec}$. There are rather large effects to be explained in terms of differential latencies of transient-channel responses, since the intensities responsible for this differential latency are not very different from each other. A second difficulty is that, since the effects of stimulus duration and stimulus luminance upon persistence duration are additive, as noted above, the two effects presumably arise at different stages in the visual system; if so, and if one 
accepts the argument (set out below) that the duration effect occurs because of the influence of stimulus duration upon the latency of transient channels, then it is difficult to claim at the same time that the luminance effect is due to the influence of stimulus luminance upon the latency of transient channels. Third, if it turns out to be the case that the inverse intensity effect is confined to mesopic intensity levels, this will strongly support a photoreceptor explanation of the effect. Thus, although it is certainly not out of the question that a relationship between transient-channel latency and stimulus intensity contributes to, or even entirely explains, the inverse intensity effect, this is not a possibility I will pursue here. Instead, I will concentrate on the implications of transient channel action for the inverse duration effect.

Any explanation of the relationship between stimulus duration and persistence duration is made difficult by the problem that we do not know whether relatively long stimuli have any visible persistence at all, though we do know that, up to some critical stimulus duration, persistence duration is inversely related to stimulus duration, while there is no effect of stimulus duration beyond this critical value. For stimuli below the critical duration, the inverse relationship between persistence duration and stimulus duration could be attributed to an effect of stimulus duration upon the latency of the inhibitory activity generated by stimulus offset: if increasing the stimulus duration reduced this latency, then an inverse relationship between stimulus duration and persistence duration would result. One objection to this proposal is that it offers no explanation of why, provided that stimulus intensity is not too low, the sum of stimulus duration and persistence duration is constant, that is, the slope of the function relating persistence duration to stimulus duration is -1.0. Any negative slope would be consistent with the offset-inhibition proposal. If an inhibitory effect were generated by stimulus onset, and if this effect had some latency (e.g., $130 \mathrm{msec}$ ), then persistence would be terminated at a fixed time $(130 \mathrm{msec})$ after stimulus onset, which would produce a slope of -1.0 ; but inhibition generated by stimulus onset is not an attractive idea, whereas inhibition generated by stimulus of fset is.

Evoked potential data reported by Servière, Miceli, and Galifret (1977b) may perhaps be relevant here. They measured the latency of the first positive peak of the cortical response evoked by stimulus onset $\left(\mathrm{P}_{1}-\mathrm{on}\right)$ and also the latency of the first positive peak of the cortical response evoked by stimulus offset $\left(P_{1}\right.$-off). The stimuli were 5-deg centrally fixated yellow disks of light; stimulus duration ranged from 2 to $500 \mathrm{msec}$, and the luminance at each duration was adjusted so that all durations looked equally bright. In practice, this meant that luminance was re- ciprocal to duration up to about $20 \mathrm{msec}$, that it decreased as duration increased in the range $20-50 \mathrm{msec}$, but not reciprocally, and that it was constant for durations in the range $50-500 \mathrm{msec}$.

Under these conditions, $\mathbf{P}_{\mathbf{1}}$-on latency was independent of stimulus duration, but $\mathbf{P}_{1}$-off latency decreased linearly as a function of stimulus duration, with a slope of -.725 , up to a duration of about $60 \mathrm{msec}$. Thereafter $P_{1}$-off latency was independent of stimulus duration. Since, throughout almost all of the duration range $0-60 \mathrm{msec}$, luminance was decreasing as duration was increasing, the slope of -.725 will underestimate the true slope of the relationship between stimulus duration and $P_{1}$-off latency if stimulus luminance has any effect on $P_{1}$-off latency over and above an effect due to total stimulus energy.

If we adopt the proposal of Kulikowski (1974, 1976) that $P_{1}$-off latency reflects the response of transient channels to stimulus offset, then these data show that the latency of this response is inversely related to stimulus duration (as proposed above), at least up to some critical duration (here about $60 \mathrm{msec}$ ), and is thereafter independent of stimulus duration. It is even possible that, if equal-luminance stimuli are used, the slope of this function may be -1.0 , which would indicate that, when the $\mathrm{P}_{1}$-on response has occurred, a fixed minimum time must elapse before the $\mathrm{P}_{1}$-off response can occur, so that, if stimulus duration is less than this minimum period, the point in time at which the $\mathrm{P}_{1}$-off response occurs will be independent of the point in time at which stimulus offset occurs. In this case, if the $\mathrm{P}_{1}$-off response is responsible for the termination of visible persistence, Efron's result will be obtained: All stimuli below a critical duration will appear to last for a time equal to that critical duration, regardless of the physical stimulus duration, whereas all stimuli above this critical duration will appear to last for a constant amount longer than their physical duration. This constant amount would depend upon the minimum latency of the $P_{1}$-off response, although it would not necessarily be equal to that latency, since termination of visible persistence might not be simultaneous with the $P_{1}$-off response, even if it is produced by this response.

In an accompanying paper (Servière et al., 1977a), a psychophysical study using precisely the same equipment and stimuli is described. Unfortunately for the purposes of this paper, it is not clear whether or not this study investigated visible persistence. The subject's task was to decide whether each stimulus was "instantaneous" or "durable." If all stimuli up to some critical duration produced a constant minimum perceptual duration, then all of these will be considered "instantaneous"; beyond this point, perceptual duration will be supraminimal and a "durable" response will be produced. Provided that the 
slope of the function relating persistence duration to stimulus duration is -1.0 up to the critical duration, and thereafter 0.0 , one can interpret the "instantaneous" response as indicating that, for this stimulus, the duration was at or below the critical duration, that is, was in the range at which duration of visible persistence is influenced by stimulus duration.

Experienced subjects produced 50\% "instantaneous" responses at a stimulus duration of about $65 \mathrm{msec}$; inexperienced subjects at about $115 \mathrm{msec}$. Similar results were obtained with a four-alternative forced-choice task.

A zero percentage of "instantaneous" responses would be produced at all durations longer than the maximum value of the critical duration, that is, beyond the longest duration for which an effect of stimulus duration on visible persistence occurs. The minimum duration yielding such zero percentages was $140 \mathrm{msec}$ for experienced subjects and $190 \mathrm{msec}$ for inexperienced subjects.

Servière et al. (1977a) compared their electrophysiological and psychophysical results by suggesting that the existence of an irreducible minimum time between $P_{1}$-on and $P_{1}$-off corresponds to the existence of an irreducible minimum duration of perception. There was reasonable agreement between the function relating stimulus duration to the interval between $P_{1}$-on and $P_{1}$-off and the function relating stimulus duration to the percentage of "instantaneous" responses.

Although the relationship of the instantaneous/ durable judgment task to the concept of visible persistence is a little obscure, the evoked-potential results reported by Servière et al. (1977b) are of direct relevance here if they can be taken as evidence for the view that, when the latency of response of transient channels to stimulus offset is measured at various stimulus durations, the resulting function has a steep negative slope (perhaps as steep as -1.0) up to some critical duration, and thereafter has zero slope. If this were so, and if the transient-channel response to stimulus offset were responsible for the termination of visible persistence, then the results of experiments which measure the relationship between persistence duration and stimulus duration could be explained.

Since transient cells are especially sensitive to low spatial frequencies, a low-spatial-frequency stimulus would have short visible persistence (the effect obtained by Meyer \& Maguire, 1977) for two possible reasons: The original sustained-cell response would not be very great (so would presumably not persist very long), and the offset-inhibition effect would be strong because the stimulus strongly affects the transient cells responsible for this effect.

Allport (1970) found that larger values for persistence duration occurred when subjects were asked to count the number of radii present in an apparent rotating fan of radii than when subjects were asked to estimate the angle formed by the leading and trailing edges of the fan. He considered the possibility, discussed above, that subjects might be capable of influencing the duration of their visible persistences in response to variations in the difficulty of the task demanded of them. An alternative possibility, however, is that the effect occurred because the radii-counting task required the use of highspatial-frequency channels, which have long persistence times, whereas the angle-estimation task could be carried out with channels sensitive to moderate spatial frequencies, and these have shorter persistence times. The idea that, when a visual stimulus has many Fourier components, the results one obtains in a psychophysical experiment will depend upon which Fourier components the subject is using to make his judgments was suggested by Carpenter and Ganz (1972). They used a vertical 1-cycle/deg square-wave grating as a target, and vertical sinusoidal gratings of various frequencies as backward masks. When the subject's task was to detect the presence or absence of the target, a task which would rely largely on the amplitude of the fundamental of the target, maximal masking was obtained with a 1-cycle/deg sinusoidal mask. However, when the subject's task was to detect the presence or absence of a misalignment between the upper and lower halves of the target, maximal masking was obtained with a 3-cycle/deg sinusoidal mask, presumably because the third harmonic of the target, the frequency of which would be 3 cycles/deg, is of critical importance for the precise information about edge location needed to perform alignment judgments. The effect of task type upon maximal masking frequency is analogous to the effect in Allport's experiment of task type upon maximal duration of persistence; different spatial frequencies have different persistence durations, so the persistence a subject demonstrates will be determined by which spatial frequencies he is using. Findings to the effect that the more difficult a perceptual task, the longer is the critical duration produced by it could be explained in the same way if the more difficult tasks require higher spatial frequencies. For example, Hunter and Sigler (1940) found that critical duration for identifying single dots was less than $100 \mathrm{msec}$, whereas for identifying multiple dots it was about 300 msec; and Kahneman and Norman (1964) showed that critical duration was larger for an identification task than for brightness discrimination.

If activity in sustained channels dies away after stimulus offset even if there is no inhibition by transient channels, it is possible, in principle, for there to be circumstances in which the sustained activity dies away before inhibition can have its effect; this will happen whenever inhibitory latency is 
greater than the decay time of sustained channels. This could be the explanation of the finding of Bowen et al. (1974), that at very low luminances stimulus duration has almost no effect on persistence duration: Perhaps, at such low luminances, the latency of the inhibitory effect, even for the longest lasting stimuli, exceeds the spontaneous decay times of sustained channels. If this were so, stimulus duration, which exerts its effect on persistence duration only via its influence on latency of inhibition, could not have any influence on persistence duration. As luminance is increased from this level, inhibitory latency will be reduced, and the proportion of occasions on which persistence is terminated by inhibition, rather than decay, will increase. Thus, as stimulus luminance increases, so will the influence of stimulus duration; hence, the steepening slope of the function relating stimulus duration to persistence duration as stimulus luminance increases, as observed by Bowen et al. (1974).

The inhibitory effects discussed here are effects of transient cells upon sustained cells. Similar ideas, involving inhibition of transient cells by other transient cells, have been invoked by Phillips and Singer (1974), Singer (1977), and Singer and Phillips (1974), to explain the results of experiments on visual memory. Although their work does not directly involve visible persistence, it merits discussion here nevertheless, for the reasons I give below.

\section{The Work of Phillips and Singer}

In the psychophysical studies described by Phillips and Singer (1974), each trial consisted of the brief presentation of a complex pattern of small lights, followed, after a brief interstimulus interval, by the same pattern, or by the same pattern plus an extra light (an "appearance'), or by the same pattern minus one light (a "disappearance"). The subject's task was to judge whether the two patterns were the same or different. This is not necessarily an experiment on visible persistence (since the task does not require that the first stimulus be visible when the second occurs) or on iconic memory (since a decaying informational trace is not necessarily involved). However, the results are still of interest to the present discussion.

Phillips and Singer proposed that subjects perform this task as follows. An appearance is detected because the ON-response to the new stimulus is detectably different from the ON-responses to all the other stimuli in the second pattern, which are merely reappearing. This difference arises because OFFresponses to the first pattern inhibit ON-responses to the second, and this will occur for every element present in both patterns. The new element, the only one not subject to this inhibition, will produce an ON response with shorter latency and higher amplitude, and the existence of one such abnormal ON-response allows the subject to determine that the two patterns on this trial are not identical: one element of the second pattern was not in the first.

This analysis of the way in which subjects detect appearances was confirmed by investigating how performance was affected by such stimulus parameters as first-pattern duration, interstimulus interval, and second-pattern duration, and showing that these effects were predictable from the results of neurophysiological studies of the mutual inhibition of $O N$ and OFF cells in the lateral geniculate. As firstpattern duration is increased, the strength of the inhibition generated by its offset is increased; hence, the one uninhibited element of the second pattern will stand out more clearly from its inhibited neighbors; therefore, detection of appearance should improve as first-pattern duration increases. This was so in the psychophysical experiments. As interstimulus interval increases, inhibition generated by the offset of the first pattern diminishes; so performance should decline as interstimulus interval increases, and this was so also in the psychophysical experiments. Finally, the inhibitory effects of first-pattern offset grow weaker as second-pattern duration is increased. This means that, as the second-pattern duration increases, the new element will stand out less clearly from its neighbours, so performance will worsen. This result, though counterintuitive, did occur.

Turning now to the way in which subjects detect disappearances, this is done because the OFF-response to the disappearing element is not cut off by an ONresponse to reappearance, as is the case with all the other elements, all of which reappear. Detection that one OFF-response differs from all the others allows the subject to determine that the two patterns are not identical: one element of the first pattern did not recur in the second. As first-pattern duration decreases, the latency of the OFF-response is greatly increased: if the second pattern is presented within this latency period, there is no OFF-response (except, of course, to the one element that is absent from the second pattern). Hence, the counterintuitive prediction is made that reducing the duration of the first pattern will actually improve performance; and it did. As interstimulus interval is increased, OFFresponses diminish and so performance worsens. As second pattern-duration increases, inhibition of OFF-responses to the first pattern is strengthened, so performance improves.

I have discussed this work in some detail for two reasons. First of all, it is a remarkable tour de force as a neurophysiological explanation of psychophysical results (or, equally, a psychophysical interpretation of neurophysiological results). Precisely this kind of work is needed if we are to make further progress in understanding the nerual bases of visible 
persistence: we need psychophysical and neurophysiological data from the same stimulus situations.

Secondly, although this psychophysical task does not depend upon visible persistence, many of the results obtained with this task resemble those obtained in studies of visible persistence. For example, there is an inverse relationship between first-pattern duration and accuracy of performance in the Phillips-Singer task, just as there is an inverse relationship between stimulus duration and visible persistence duration. This reinforces the view that we ought to consider the role played by $\mathrm{ON}$ - and OFFresponses and their mutual inhibition in studies of visible persistence.

Suppose, for example, that a subject decides that a stimulus has disappeared, not because sustained activity has ceased, but because the OFF-response has occurred. Singer and Phillips (1974) show that the latency of the OFF-response increases as stimulus duration decreases; hence, visible persistence (as measured by detection of stimulus offset) would increase in duration as the stimulus decreased in duration, as Briggs and Kinsbourne (1974) observed.

It is obviously plausible to interpret in this way studies of visible persistence using the onset-offset reaction time technique; but what of the other six tehcniques described earlier in this paper? Synchrony judgment could be performed by setting the OFFresponse to the test stimulus so that it is simultaneous with the ON-response to the probe. Phenomenal continuity might be attained when the ON-response to a reappearing stimulus occurs before the OFFresponse to the preceding presentation of the stimulus, although, as noted earlier, a flickering stimulus can still be phenomenally continuously present. Presumably, here ON- and OFF-responses are mediating the appearance of flicker, so something else may be responsible for the phenomenal continuity. Temporal integration of form parts might occur whenever the last form part is presented before the OFF-response to the first had occurred; a similar analysis applies to the moving-slit technique and the effects of stroboscopic illumination of a moving stimulus. Nothing useful can be said here about the stereoscopic persistence without a review of the properties of disparity-sensitive cortical neurones.

Whether the OFF-response itself directly signals stimulus offset, or does so indirectly by inhibiting the persisting activity of sustained cells, is not important for the argument here. What is important is that a straightforward explanation of the inverse duration effect is provided if we assume that the OFF-response is directly or indirectly implicated in the termination of visible persistence. Given that the interval between stimulus onset and occurrence of the OFF-response does not vary, or varies very little, as a function of stimulus duration, the duration of visible persistence as measured from stimulus offset will be an inverse function of stimulus duration.

However, the inverse luminance effect has no obvious interpretation within this framework. One might suppose that as luminance increases, amplitude of the ON-response increases, so inhibition of the OFF-response is increased and the latency of the OFF-response is extended; but this would mean that more intense stimuli would have longer visible persistences. The only way out here is the ad hoc suggestion that as luminance increases, the increasing inhibition generated by the $\mathrm{ON}$-response is more than offset by the reduction in latency of the OFF-response. Although ad hoc, this might be true, and certainly is open to direct neurophysiological verification. An alternative is to ascribe the inverse luminance effect to events occurring at the photoreceptor level, as discussed earlier.

\section{Summary}

There are neural persistences in the visual system from the photoreceptor level on up. Both rods and cones continue to signal for some time after stimulus offset, rod activity persisting longer than cone activity. Such photoreceptor persistence may be one of the neural bases for visible persistence. If one accepts claims that cones inhibit rods, it is even possible to explain why, at least at mesopic light levels, there is an inverse relationship between stimulus luminance and duration of visible persistence.

Sustained cells in the visual pathway also exhibit poststimulus persistence. If visible persistence ceases when this persistence is cut off by inhibition via the OFF-response, or alternatively if the OFF-response itself directly signals stimulus offset, then one can explain the inverse relationship between stimulus duration and persistence duration. The OFF-response has a minimum latency as measured from the onset of the stimulus: Therefore, if visible persistence is measured from stimulus offset, its duration will be inversely related to stimulus duration, up to a duration equal to the minimum off-response latency; beyond this point, persistence duration will be independent of stimulus duration.

These claims about the neural bases of visible persistence can be investigated in very straightforward ways, by studies which follow the lead of Phillips and Singer (1974) and Singer and Phillips (1974) in making both psychophysical and neurophysiological studies using the same stimulus setup. In this way, any theory which makes detailed claims about how the psychophysics and the neurophysiology will relate to each other can be rigorously tested. Until such work is forthcoming, it remains merely plausible to claim that visible persistence is produced by photoreceptor persistence, and also by persistence in the visual pathways. It must also be kept in mind that Engel (1970) has shown a cortical form of visible persistence. Thus, it is inappropriate to ask where 
the neural locus of visible persistence is: the loci are multiple, as all stages of the visual system from retina to cortex are involved.

\section{WHAT IS ICONIC MEMORY?}

So far in this paper, I have said a considerable amount about what iconic memory is not: it is not visible persistence, or an afterimage, or neural persistence at any stage of the visual system from retina to cortex. Not much has been said, however, about what iconic memory is. At the beginning of the paper, I drew a distinction between theories as to the nature of iconic memory and experimental methods used to investigate iconic memory; and later I argued that the method which defines iconic memory is the partial report technique. This is not to say that, if a partial report advantage which diminishes as cue delay increases is obtained in an experiment, then this experiment must be dealing with iconic memory. As I have argued, an afterimage is sufficient to generate such effects; but not necessary, since these effects have been observed in experiments where stimuli were not intense enough to produce afterimages. Thus, the existence of a partial-report superiority is not sufficient as a criterion that one is dealing with iconic memory. It is not necessary either. Many experiments using a single-item probe technique, from Averbach and Coriell (1961) onwards, have not included a full-report condition, so could not produce evidence for a partial-report superiority; yet these are clearly experiments on iconic memory.

\section{The Experimental Criterion for Iconic Memory}

If a partial-report advantage is neither necessary nor sufficient for identifying an experiment as one which is studying iconic memory, then what criterion can we use to decide whether or not we are dealing with iconic memory? I propose the following. A visual display consisting of a number of items is presented. At or after display offset, the subject is given a cue which defines a subset of the display items. The subject's task is to report as many of the items in this subset as he can. If his performance declines as the interval between display and cue increases, down to some asymptotic level, and remains at that level for longer cue delays, then the display was represented in iconic memory and the cue used to sample selectively the contents of iconic memory. A qualification must be added: The display luminance must have been too low to generate an afterimage. This qualification is inelegant; but what alternative do we have? Afterimages certainly will produce a cue delay effect in partial report experiments, even if there were no such thing as iconic memory. The question is whether a cue delay effect in partial report experiments occurs in the absence of afterimages. If afterimages require stimuli intense enough to saturate the rods, as Sakitt (1976b) claims, then a cue delay effect does occur in the absence of afterimages, and it is iconic memory which is responsible.

Although a partial report superiority is not a necessary condition for iconic memory experiments (given the many single-letter cue experiments which do not use a full-report condition), it may, given that afterimages are absent, be claimed as a sufficient condition; but the problem here is output interference. If the cued items are a row of four from a threerow display, and a subject achieves, say, $80 \%$ correct in partial report, he must report 9.6 items in full report if there is to be no partial report advantage. And it can be argued that reporting 9.6 items is too difficult a task; the reporting of the early items may destroy the memory representations of items not yet reported. This would produce a partial report superiority, but not because of any property of iconic memory. However, if a partial report superiority owes its existence to output interference, it should not diminish rapidly in size down to zero as the cue delay increases from zero to, say, 500 msec. Thus, an adequate defence against the problem of output interference is to show that one's partial report superiority is strongly affected by cue delay.

Yet another qualification is needed here. If full report diminishes as report delay increases, then a diminution of partial report may not indicate the existence of iconic memory. Under normal circumstances, this is no problem, because full report is not usually affected by delaying the report. However, with a very large subsidiary memory load, Sakitt and Appelman (1978) did obtain a delay effect on full report. They concluded that "the existence of a partial report superiority and/or a decaying partial report curve does not necessarily imply the existence of an icon or visual storage." This is true, but perhaps not of vital importance, since their point does not apply as long as full report does not show a report delay effect, and it is only in exceptional circumstances (e.g., large subsidiary memory load) that full report does show a report delay effect. In the usual circumstances, there is no such delay effect on full report, so a decaying partial report does provide evidence for iconic memory (provided afterimages are not present).

\section{What Decays?}

The notion that iconic memory is like a steadily fading afterimage rests on the idea that the decay of iconic memory consists of the gradual fading of each item in this form of memory. But it is by no means clear that this is the correct way of conceptualizing decay in iconic memory. Dick $(1969,1970)$ has provided data which might suggest a different conceptualization. In his experiment, full report and 
partial report trials were randomly intermixed, with varying cue delays. When partial report was by position (top or bottom row) or color (black or red letters), performance decreased as cue delay increased. When partial report was by stimulus category (letter or digit), partial report performance was constant across cue delays. The usual interpretation (e.g., Coltheart, 1972, 1975a) of this kind of result is that it indicates that iconic memory contains information about spatial location, color, and other such physical attributes, but does not contain information about "semantic" or "higher order" attributes such as whether an item is a letter or a digit.

An alternative explanation is that what decays in iconic memory is information about such physical attributes as spatial location or color, while information about the identity of an item is also represented in iconic memory but does not decay. Such a view has been suggested by Townsend (1973). First, she observed that in a single-letter cue experiment of the kind introduced by Averbach and Coriell (1961), performance declined as cue delay increased, but the proportion of incorrect reports of letters which had not been present in the display (intrusion errors) did not increase as cue delay increased. If the identity of display items decayed over time, one would expect an increasing proportion of intrusion errors as cue delay increased. The absence of this trend thus is consistent with the idea that identity information does not decay, while spatial information does: As cue delay increases, more and more often what one knows about a certain letter is that it was in the display but not where it was in the display. A second experiment reported by Townsend (1973) supports this view. When the postexposure cue requested solely identity information (the cue was a single letter, at various cue delays, and the subject's task was to decide whether or not the cue letter had been in the display), performance was constant across the cue delays.

If identity information does not decay but spatial information does, one can predict not only that the rate of intrusion errors should not vary as a function of cue delay, but also that the rate of transposition errors (reporting an item which was in the display but not in the cued position) should increase as cue delay increases. This prediction was confirmed by Di Lollo (1978).

There are at least two difficulties with the discussion here. First, the experiments of Dick (1969) and Townsend (1973) produced some unusual results. In Dick's experiments, not only did partial report decline with cue delay in the spatial location and color conditions, but so did full report; in the stimulus category condition, neither form of report declined with cue delay. In Townsend's experiment, performance showed no cue-delay effect when the cue was long lasting $(900 \mathrm{msec})$. Second, it is by no means clear than one must accept that these results show that identity information does not decay in iconic memory: it could well be that in the identity tasks of Dick and of Townsend, selection and report is not from iconic memory, but from posticonic "durable storage." If spatial information is poorly represented in durable storage, the increasing reliance on durable storage as cue delay increases could produce an increase in transposition errors but no increase in intrusion errors. A detailed discussion of these problems is needed, but will not be offered here. I merely note that the ideas proposed by Dick (1969), Di Lollo (1978), and Townsend (1973), suggest the following: The identity of an item is stored rapidly and in a stable form early in the lifetime of a display, while physical attributes of the item are registered with more difficulty and in an unstable decaying form. To argue in this way runs counter to the conventional view of iconic memory as a buffer store which prolongs the availability of unprocessed visual information so as to give relatively slow-acting identification mechanisms more time to do their job. Furthermore, the view that iconic memory is like a photograph which loses contrast over time-like an afterimage, in fact-is also inconsistent with the concept that identity information does not decay while physical information does.

At the beginning of this paper, I outlined the usual view of iconic memory-namely, that it is a high-capacity fast-decay buffer store from which items either are preserved by transfer to durable storage or else are lost by decay (integral decay: that is, identity and physical information about an item are lost as a whole unit). I also noted that this conception of iconic memory might be incorrect. I would like to conclude by outlining an alternative conception. I will make no attempt to adjudicate between these two views of iconic memory; such adjudication may not even be possible, given what data we currently have about iconic memory. However, it seems appropriate here to conclude by sketching the alternative approach to iconic memory, because this approach is only conceivable once one rejects wholeheartedly the identification of iconic memory with any form of visible persistence, and the main point of this paper is to urge such a rejection. I should also point out that this sketch is derived to a very substantial extent from ideas put forward by Allport (1977, Note 4, Note 5) and by van der Heijden (1978), although they may well regard what I have to say here as being a caricature rather than a sketch.

\section{Iconic Memory and Lexical Access}

The concept of an internal dictionary or internal lexicon, introduced within the context of a discussion 
of dichotic listening data by Treisman (1960), is currently widely used in theoretical analyses of reading (e.g., Coltheart, 1978a, 1978b; Coltheart, Davelaar, Jonasson, \& Besner, 1977). The reader is considered to identify printed symbols (words, numbers, and letters) or spoken items by referring to their entries within the internal lexicon; this process is lexical access.

A major problem for any model of reading (or speech perception) which includes a component corresponding to a lexicon is as follows. A printed word can appear in innumerable different physical forms, but surely this does not mean that it has innumerable different lexical entries? This would be absurdly uneconomical. Surely, there is only a single lexical entry for a given word, an entry which is accessed regardless of the particular physical appearance of the word on a particular occasion. Certainly, this is the assumption that is usually made; but if it is made, then information about the particular physical appearance of a word on a particular occasion is not retained as a consequence of the process of lexical access. If we see BOOK written in red and TABLE written in green, and observe the consequences this has for lexical access, we will note that the lexical entries for "book" and "table" have been accessed, and so these words must have been present. But where is the information that one word was red and one green-let alone the information about which was red and which green? Yet, manifestly, it is trivially easy for a reader to answer a question such as "Which word was written in green?" Indeed, the word and its ink color, rather than being difficult to associate, are difficult to dissociate: the Stroop effect shows this.

Exactly similar issues arise in connection with speech perception. We do not have excessive difficulty in deciding which of two words was spoken in a high-pitched voice and which in a low-pitched voice; indeed, dissociation is the difficulty here, since Stroop effects are present in this situation too (Cohen \& Martin, 1975).

The theoretical problem here is how episodic and semantic memory (Tulving, 1972) can be coordinated. An episodic description of a word tells us all about its particular physical properties on a given occasion, but nothing about its properties which are independent of particular occasions (such as its meaning). A description of the word in terms of semantic memory provides information about the word's enduring properties, but not about how it was manifested on any particular occasions. How do we coordinate these two sources of memory for a word? No theory yet provides a satisfying answer to this question; nor will this paper. However, I will argue that such coordination is evidently not a difficult task for us to accomplish; in some way, we can temporarily tag lex- ical entries with episodic information. The question of how we do it is completely begged here; but I will follow Allport (1977) in proposing that we do do it, and that this tagging is sensory memory. For the case of vision, then, setting up an iconic memory consists of temporarily attaching various forms of physical information to a permanently existing entry in the internal lexicon. This attachment is a rapid, automatic process with unlimited capacity; but the attached information decays rapidly. If it is to be preserved, a mechanism, which I will call a lexical monitor, must operate upon the physical information, transforming it into some more durable form. The operation it performs I will term "lexical stabilization."

The attached physical information decays rapidly; but suppose it is not needed? Suppose the task is merely to report all the items that had been in a display, regardless of their physical properties? This amounts to identifying which entries in the lexicon have been accessed. Clearly, an observer's ability to do this is not unlimited, since observers can report only about four or five items on the average from a brief alphanumeric display. What is the origin of this limitation? Perhaps it is the decay of excitation in a lexical entry, as envisaged, for example, in the logogen model of the lexicon (Morton, 1969). In the absence of lexical stabilization, even information about whether a lexical entry has been accessed decays away. When numerous lexical entries are simultaneously accessed, a lexical stabilization process which is serial or which is parallel with limited capacity may be able to stabilize only a subset of these items: the others decay away and so cannot be reported. Although the latter items cannot be reported, they have nevertheless achieved lexical access, and hence can generate semantic facilitation effects: hence, the finding that unreported items can have effects dependent upon their meanings (e.g., Andreewsky, Deloche, \& Kossanyi, in press; Allport, Note 4).

On this view, the partial report superiority occurs because the choice of lexical entries for lexical stabilization can be controlled by the nature of the physical information attached to these lexical entries. The lexical monitor can respond to the cue by stabilizing that subset of items specified by the cue (this corresponds to the concept of "selective readout"). In the absence of such a cue and also before the cue is decoded, stabilization may be nonselective ("nonselective readout'').

If we assume that the decay of activation of a lexical entry is relatively slow compared to the decay of attached physical information, then identity information will not show the same decline with cue delay as will spatial location or other such physical information; this finding was discussed above.

Any theory of iconic memory must also offer an account of how visual masking works. Turvey (1973) 
distinguished between peripheral and central visual masking. Peripheral masking involves integration within monocular visual channels, so depends upon the energy of the target relative to the mask and does not occur dichoptically. Central masking involves interruption of target processing, occurs dichoptically, and depends upon feature-similarity of target and mask. By analogy with the views of Allport (1977, Note 4) and Marcel (Note 6), it is assumed here that central masking interrupts target processing because the pattern mask preempts the activities of the lexical monitor and, hence, interferes with the process of lexical stabilization. It was shown by Sperling (1967) that if one varies the stimulus-onset asynchrony (SOA) between an alphabetic display and a backward mask, the number of letters which can be reported from the display is a roughly linear function of SOA up to about 4 letters and an SOA of $50 \mathrm{msec}$. Further increases in SOA add only a little to the amount that can be reported. Thus, the function relating number of letters reported and SOA has two limbs, an initial steep segment and a subsequent shallow segment (for examples, see Coltheart, 1972). The limiting factor for the first segment, it is argued here, is whether lexical stabilization of an item is completed before the backward mask deflects the attention of the lexical monitor. The limiting factor for the second segment is the limited processing capacity of the monitor itself: it cannot cope adequately when required to deal with numerous lexical entries, and increases in SOA do not help much with this problem.

Data from Allport (Note 5) are important here. Within the range of SOAs producing the initial steep segment of the masking function, Allport showed that the SOA which permitted report of, say, two single letters also permitted the report of two threeletter words. Thus the processing going on during the stimulus-mask interval does not depend on the number of letters which must be processed, but the number of linguistic units-that is, the number of lexical entries. Pursuing this line of reasoning in a second experiment, Allport showed that the "rate of processing" - the number of words that can be reported at a given SOA-was not influenced by the number of letters or number of syllables in the words, but was strongly influenced by word frequency. Since lexical access time is widely assumed to depend upon word frequency, and to be independent of number of letters or syllables, these results of Allport's favor the postlexical view of iconic memory being discussed here. They are not easy to reconcile with theories of durable storage such as those of Coltheart (1972) or Sperling (1967), according to which the nonmaskable storage mode is phonological (here a syllable effect might be expected) or dual phonological/visual (here effects of both number of letters and of numbers of syllables might be expected).
Some neuropsychological data are also particularly interesting here, namely, data from the syndrome termed "attentional dyslexia" by Shallice and Warrington (1977), and studied by these authors and by Levine and Calvanio (1978). This disorder, produced by damage to the left hemisphere, has as its principal symptom an inability to report items when there are several such items present, though single-item report is unimpaired. For example, when a single letter is briefly presented with a backward mask, the patients can report the letter at SOAs which do not differ from those required by normal controls; but when three unrelated letters are to be reported, even an exposure duration of $5 \mathrm{sec}$ does not produce perfect performance. However, when these three letters constitute a word, performance is much improved (Levine \& Calvanio, 1978), paralleling Allport's results with normal subjects.

This defect is not a low-level visual difficulty, because performance is good when the patient is asked to judge whether three letters are identical or not, and also because if the patient is precued as to which of three letters from a brief display he is to report ("left," "center," or "right"' or a spatial cue, indicating one of these positions), his performance is much improved. A postcue delayed by $1 \mathrm{sec}$, on the other hand, does not assist performance: the cued letter now fares no better than it would in full report.

Within the postlexical approach to iconic memory, one can describe these patients as suffering from a defect of the lexical monitor: when more than one lexical entry is accessed, the monitor has severe difficulties carrying out lexical stabilization of all of the entries, though it can stabilize that entry specified by a precue. This difficulty exists for normal subjects too; it is merely a question of how many lexical entries need to be accessed before the abilities of the lexical monitor are taxed. For the patients, as soon as there is more than one entry to deal with, the monitor experiences difficulties; for normals, it is four or five entries.

It was shown by Shallice and Warrington (1977) that the ability of these patients to report a letter cued by color and position (it was a central, red item flanked on either side by black items) was lower when it was flanked by other letters than when it was flanked by digits. This result might be interpreted in terms of the lexical discrimination required of the monitor: if two lexical entries are (semantically) "close", the decision as to which should be stabilized so that it can be reported is more difficult than when the entries are semantically distant. The effect cannot be an output effect, since the patient can respond "four" to four dots when these are flanked by digits, but has great difficulty responding "four" to 4 when it is surrounded by digits.

The behavior of Shallice and Warrington's patients 
has its parallel in experiments with normals. Eriksen and Eriksen (1974) required their subjects to push a lever to one side when a centrally fixated letter was an $\mathrm{H}$ or $\mathrm{K}$ and to the other when the letter was an $\mathrm{S}$ or a $\mathrm{C}$. The central letter was flanked on each side by letters which were irrelevant for the response. By comparison with a control condition where the flanking letters were not drawn from the set $\mathrm{H}, \mathrm{K}, \mathrm{S}$, and $C$, responses to the central letter were faster when the flanking letters belonged to the same set and slower when they belonged to a different set. Thus, the response to $\mathrm{H}$ was fastest, for example, in $\mathrm{KHK}$, intermediate in BHB, and slowest in SHS.

The relevance of such Stroop-like interference and facilitation effects to the theory of iconic memory has been stressed by van der Heijden (1978). If there is interposed between iconic memory and identificatory mechanisms a selective stage which passes on for identification only those items meeting some physical criterion specified by a cue, then items not meeting that criterion (in the case of the Eriksens' experiment, this criterion being "center letter") would not reach the identification stage, and so no effect due to the specific identities of the flanking letters should be observed.

On the other hand, if selection is subsequent to lexical access, these effects of the flanking letters would be expected. All three letters reach their lexical entries, and it is the task of the lexical monitor to determine which lexical entry corresponds to the center letter. While this is happening, the irrelevant letters are prompting correct or incorrect responses; hence, they cause response facilitation or interference. An exact parallel to the behavior of the attentional dyslexic would be obtained if one could show, using the Eriksen and Eriksen technique, that the classification of the center letter is more impaired by flanking letters than by flanking digits, even when the letters were not from the critical set $K, H, S$, and $C$.

This discussion of iconic memory as a postlexical process has been highly speculative and, in places, extremely vague; concepts such as "lexical monitoring" and "lexical stabilization"' are far from precise. I hope, however, that the discussion has been clear enough to indicate that there genuinely do exist two very different theoretical approaches to iconic memory. According to one approach, iconic memory is a rapidly decaying high-capacity buffer store from which a subset of items can be selected for transfer to and subsequent retention in a smaller capacity but more durable storage mode; identification of items takes place subsequent to this transfer process. Items not transferred decay away and are never identified. This might be called the prelexical or precategorical view of iconic memory.

The alternative is that all items are identified, that is, reach their lexical entries. Iconic memory consists of the tagging of each of these entries with information about the physical manifestation of the word corresponding to that entry. This physical information decays away rapidly unless "lexically stabilized" by a "lexical monitor" of limited capacity. Lexical excitation itself also decays (though more slowly), and so also may require lexical stabilization. Backward masking can interfere with lexical stabilization by claiming the attention of the lexical monitor: this is how "perception without awareness" occurs. This is the postlexical or postcategorical view of iconic memory.

No systematic attempt has been made at arguing for one or another of these approaches, but, in view of the current predominance of the prelexical approach, certain results which appear to favor the postlexical approach have been described.

It is for future work to explore these issues. What is already clear now, I have argued, is that such work ought not to use the so-called "direct methods" of studying iconic memory; for there are no direct methods for studying iconic memory. What the direct methods are studying is visible persistence, and these methods have produced a wealth of information about the properties of visible persistence. We also know a considerable amount about neural persistences at various stages of the visual system and how these may be related to visible persistence. By comparison, our understanding of iconic memory is seriously inadequate. One reason for this has been, in my view, the confusion between visible and neural persistence, on the one hand, and iconic memory, on the other; and if this is so, then this paper, though largely devoted to describing what iconic memory is not, may be of some use for future attempts at finding out what it is.

\section{REFERENCE NOTES}

1. Mollon, J. Two approaches to the perceptual moment hypothesis. Paper presented at the meeting of the Experimental Psychology Society, Oxford, England, 1969.

2. Fox, R., Lehmkuhle, S., \& Shea, S. Iconic memory in stereoscopic space: Seeing without storing. Paper presented at the 18th Annual Psychonomic Society meeting, St. Louis, Mo., 1977.

3. Adelson, E. H., \& Jonides, J. The psychophysics of iconic storage. Paper presented at the Psychonomic Society meeting St. Louis, 1978.

4. Allport, D. A. Word recognition and reading. Paper presented at the meeting on Processing of Visible Language, Eindhoven, The Netherlands, 1977.

5. Allport, D. A. Word recognition and the recognition buffer. Paper presented at the meeting of the Experimental Psychology Society, London, 1973.

6. Marcel, A. J. Perception with and without awareness. Paper presented at the meeting of the Experimental Psychology Society, Stirling, Scotland, 1974. 


\section{REFERENCES}

Adelson, E. H. Iconic storage: The role of rods. Science, 1978, 201, 544-546.

Aguilar, M., \& Stiles, W. S. Saturation of the rod mechanism of the retina at high levels of stimulation. Optica Acta, 1954, 1, 59-65.

Allport, D. A. Studies in the psychological unit of duration. Unpublished PhD thesis, University of Cambridge, 1966.

Allport, D. A. Temporal summation and phenomenal simultaneity: Experiments with the radius display. Quarterly Journal of Experimental Psychology, 1970, 22, 686-701.

AllPORT, D. A. On knowing the meanings of words we are unable to report: The effects of visual masking. In S. Dornic (Ed.), Attention and performance VI. Hillsdale: Erlbaum, 1977.

Alpern, M., \& BARR, L. Durations of the afterimages of brief light flashes and the theory of the Broca and Sulzer phenomenon. Journal of the Optical Society of America, 1962, 52, 219-221.

Andreewsky, E., Deloche, G., \& Kossanyi, P. Analogies between speed reading and deep dyslexia (Towards a procedural understanding of reading). In M. Coltheart, K. Patterson, \& J. C. Marshall (Eds.), Deep dyslexia. London: Routledge and Kegan Paul, in press.

Anstis, S. M., \& Atkinson, J. Distortions in moving figures viewed through a stationary slit. American Journal of Psychology, 1967, 80, 572-585.

Arnett, J. L., \& DiLollo, V. Visual information processing in relation to age and reading ability. Journal of Experimental Child Psychology, in press.

Averbach, E., \& Coriell, A. S. Short-term memory in vision. Bell Systems Technical Journal, 1961, 40, 309-328.

Averbach, E., \& Sperling, G. Short-term storage of information in vision. In C. Cherry (Ed.), Information theory. London: Butterworth, 1961.

Banks, W. P., \& Barber, G. Color information in iconic memory. Psychological Review, 1977, 84, 536-546.

Bartlett, N. R., Sticht, T. G., \& Pease, V. P. Effects of wavelength and retinal locus on the reaction time to onset and offset stimulation. Journal of Experimental Psychology, 1968, 78, 699-701.

Bowen, R. W., Pola, J., \& Matin, L. Visual persistence: Effects of flash luminance, duration and energy. Vision Research, 1974, 14, 295-303.

Breitmeyer, B. Simple reaction time as a measure of the temporal response properties of transient and sustained channels. Vision Research, 1975, 15, 1411-1412.

Breitmeyer, B., \& Ganz, L. Temporal studies with flashed gratings: Inferences about human transient and sustained channels. Vision Research, 1977, 17, 861-865.

Breitmeyer, B., \& Julesz, B. The role of on and off transients in determining the psychophysical spatial frequency response. Vision Research, 1975, 15, 411-415.

Briggs, G. G., \& Kinsbourne, M. Visual persistence as measured by reaction time. Quarterly Journal of Experimental Psychology, 1972, 24, 318-325.

Carpente R, P. A., \& Ganz, L. An attentional mechanism in the analysis of spatial frequency. Perception \& Psychophysics, $1972,12,57-60$.

Clark, S. E. Retrieval of color information from preperceptual memory. Journal of Experimental Psychology, 1969, 82, 263-266.

Cleland, B. G., Levick, W. R., \& Sanderson, K. J. Properties of sustained and transient ganglion cells in the cat retina. Journal of Physiology, 1973, 228, 649-680.

Cohen, G., \& Martin, M. Hemisphere differences in an auditory Stroop task. Percpetion \& Psychophysics, 1975, 17, 79-83.

CoHene, L. S. Iconic memory of dot patterns: Preliminary report. Perceptual and Motor Skills, 1975, 41, 167-170.

Coltheart, M. Visual information-processing. In P. C. Dodwell, (Ed.), New horizons in psychology. Harmondsworth, England: Penguin Books, 1972.
Coltheart, M. Colour-specificity and monocularity in the visual cortex. Vision Research, 1973, 13, 2595-2598.

Coltheart, M. Iconic memory: A reply to Professor Holding. Memory \& Cognition, 1975, 3, 42-48. (a)

Coltheart, M. Doubts about iconic memory: A reply to Holding. Quarterly Journal of Experimental Psychology, 1975, 27, 511-512. (b)

Coltheart, M. Contemporary models of the cognitive processes. I: Information input and storage. In V. Hamilton \& M. D. Vernon (Eds.), The development of cognitive processes. London: Academic Press, 1977.

Coltueart, M. Lexical access in simple reading tasks. In G. Underwood (Ed.), Strategies of information-processing. London: Academic Press, 1978. (a)

Colthe art, $M$. The internal lexicon and its access during reading. In J. P. Sutcliffe (Ed.), Conceptual analvsis and method in psychologv. Sydney: University Press, 1978. (b)

Coltheart, M., Davelaar, E., Jonasson, J. T., \& Besner, D. Access to the internal lexicon. In S. Dornic (Ed.), Attention and performance VI. New York: Erlbaum, 1977

Colthfart, M., Lfa, C. D., \& Thompson, K. In defence of iconic memory, Quarterly Journal of Experimental Psychology, 1974, 26, 633-641.

Cumming, G. D. Visual perception and metacontrast at rapid input rates. Unpublished D.Phil. thesis, University of Oxford, 1972.

Demkiw, P., \& Micharls, C. Motion information in iconic memory. Acta Psychologica, 1976, 40, 257-274.

Dick, A. O. Relations between the sensory register and shortterm storage in tachistoscopic recognition. Journal of Experimental Psychology, 1969, 82, 279-284.

Dick, A. O. Visual processing and the use of redundant information in tachistoscopic recognition. Canadian Journal of Psychology, 1970, 24, 133-141.

Di LoLLo, V. Temporal characteristics of iconic memory. Nature, $1977,267,241-243$.

Di LoLLo, V. On the spatio-temporal interactions of brief visual displays. In R. H. Day \& G. V. Stanley (Eds.), Studies in perception. Perth: University of Western Australia Press, 1978.

Di LolLo, V. Temporal integration in visual memory. Journal of Experimental Psychology: General, in press.

Di Lollo, V., \& Wilson, A. E. Iconic persistence and perceptual moment as determinants of temporal integration in vision. Vision Research, 1978, 18, 1607-1610.

Dixon, N. F., \& Hammond, J. The attenuation of visual persistence. British Journal of Psychology, 1972, 63, 243-254.

Erron, R. Stereoscopic vision-I: Effect of binocular summation. British Journal of Ophthalmology, 1957, 41, 709-730.

Efron, R. The relationship between the duration of a stimulus and the duration of a perception. Neuropsychologia, 1970, 8, 37-55. (a).

Efron, R. The minimum duration of a perception. Neuropsychologia, 1970, 8, 57-63. (b)

Erron, R. Effect of stimulus duration on perceptual onset and offset latencies. Perception \& Psychophysics, 1970, 8, 231-234. (c)

Erron, R. An invariant characteristic of perceptual systems in the time domain. In S. Kornblum (Ed.), Attention and performance IV. New York: Academic Press, 1973.

Efron, R., \& LeE, D.N. The visual persistence of a moving stroboscopically illuminated object. American Journal of Psychology, 1971, 84, 365-375.

ENGEL, G. R. An investigation of visual responses to brief stereoscopic stimuli. Quarterly Journal of Experimental Psychology, 1970, 22, 148-160.

Eriksen, B. A., \& Eriksen, C. W. Effects of noise letters upon the identification of a target letter in a nonsearch task. Perception \& Psychophysics, 1974, 16, 143-149.

Eriksen, C. W., \& Collins, J. F. Some temporal characteristics of visual pattern perception. Journal of Experimental Psychology, 1967, 74, 476-484. 
Eriksen, C. W., \& Coluins, J. F. Sensory traces versus the psychological moment in the temporal organization of form. Journal of Experimental Psychology, 1968, 77, 376-382.

Erjksen, C. W., \& Rohrbaugh, J. W. Visual masking in multielement displays. Journal of Experimental Psychology, 1970, 83, 147-154

Fain, G. L., \& Dowling, J. E. Intracellular recordings from single rods and cones in the mudpuppy retina. Science, 1973 , 180, 1178-1181.

Fehrer, E., \& RAAB, D. Reaction time to stimuli masked by metacontrast. Journal of Experimental Psychology, 1962, 63, 143-147.

GELDARD, F. The human senses. New York: Wiley, 1953.

Haber, R. N., \& Nathanson, L. S. Post-retinal storage? Some further observations on Parks' camel as seen through the eye of a needle. Perception \& Psychophysics, 1968, 3, 349-355.

Haber, R. N., \& Standing, L. Direct measures of short-term visual storage. Quarterly Journal of Experimental Psychology, $1969,21,43-54$

Haber, R. N., \& Standing, L. Direct estimates of the apparent duration of a flash. Canadian Journal of Psychology, 1970, 24, 216-229.

Hogben, J. H., \& Dı Lollo, V. Perceptual integration and perceptual segregation of brief visual stimuli. Vision Research, $1974,14,1059-1069$

Holving, D. H. Guessing behavior and the Sperling store. Quarterly Journal of Experimental Psychology, 1970, 22, 248256.

Holving, D. H. Brief visual memory for English and Arabic letters. Psychonomic Science, 1972, 28, 241-242.

Holding, D. H. Recognition tests of visual information storage. British Journal of Psychology, 1973, 64, 9-16.

Holving, D. H. Doubts about iconic memory: A reply to Coltheart, Lea and Thompson. Quarterly Journal of Experimental Psychology, 1975, 27, 507-509. (a)

Holding, D. H. Sensory storage revisited. Memory \& Cognition, 1975, 3, 31-41. (b)

Hunter, W. S., \& Sigler, M. The span of visual discrimination as a function of time and intensity of stimulation. Journal of Experimental Psychology, 1940, 26, 160-179.

Julesz, B. Foundations of cyclopean perception. Chicago: University of Chicago Press, 1971.

Julesz, B., \& Chiarucci, E. Short-term memory for stroboscopic movement perception. Perception, 1973, 2, 249-260.

Kahneman, D., \& Norman, J. The time-intensity relation in visual perception as a function of observer's task. Journal of Experimental Psychology, i964, 68, 215-220.

KeEle, S. W., \& Ch.ASE, W. G. Short-term visual storage. Perception \& Psychophysics, 1967, 2, 383.386.

KULIKowsKi, J. J. Human averaged occipital potentials evoked by pattern and movement. Journal of Physiology, 1974, 242, 70-71.

KULikowski, J. J. Visual evoked potentials as a measure of visibility. In J. E. Desmedt (Ed.), Visual evoked potentials in man: New developments. Oxford: Clarendon Press, 1976.

Kulikowski, J. J., \& Tolyurst, D. J. Psychophysical evidence for sustained and transient channels in human vision. Journal of Physiology, 1973, 232, 149-163.

LEGRAND, Y. Light, colour and vision. London: Chapman and Hall, 1957

Levine, D. N., \& Calvanio, R. A study of the visual defect in verbal alexia-simultanagnosia. Brain, 1978, 101, 65-81.

Lowe, D. G. Temporal aspects of selective masking. Quarterly Journal of Experimental Psychology, 1975, 27, 375-385.

Mackworth, J. The duration of the visual image. Canadian Journal of Psychology, 1963, 17, 62-81.

Makous, W., \& Boothe, R. Cones block signals from rods. Vision Research, 1974, 14, 285-294.

Mayzner, M. S., \& Tresselt, M. E. Visual information processing with sequential inputs: A general model for sequential blanking, displacement, and overprinting phenomena.
Annals of the New York Academy of Sciences, 1970, 169, 599-618.

MEYER, G. E. The effects of color-specific adaptation on the perceived duration of gratings. Vision Research, 1977, 17, 51-56.

Meyer, G. E., Lawson, R. L., \& Cohen, W. The effects of orientation-specific adaptation on the duration of short-term visual storage. Vision Research, 1975, 15, 569-572.

Meyer, G. E., \& Maguire, W. M. Spatial frequency and the mediation of short-term visual storage. Science, 1977, 198 , 524-525.

Mollon, J., \& Polden, P. On the time constants of tachistoscopes. Quarterly Journal of Experimental Psychology, 1978, 30, 555-568.

Morton, J. Interaction of information in word recognition. Psychological Review, 1969, 76, 165-178.

Neisser, U. Cognitive psychology. New York: AppletonCentury-Crofts, 1967.

Norman, R. A., \& Werblin, F. S. Control of retinal sensitivity: I. Light and dark adaptation of vertebrate rods and cones. Journal of General Physiology, 1974, 63, 37-61 .

OGLE, K. N. Stereoscopic depth perception and exposure delays between images in the two eyes. Journal of the Optical Society of America, 1963, 53, 1296-1304.

Parker, D. M., \& Salzen, E. A. Latency changes in the human visual evoked response to sinusoidal gratings. Vision Research, $1977,17,1201-1204$

Parks, T. E. Post-retinal visual storage. American Journal of Psychology, 1965, 78, 145-147.

Pease, V. P., \& Sticht, T. G. Reaction time as a function of onset and offset stimulation of the fovea and periphery. Perceptual and Motor Skills, 1965, 20, 549-554.

PenN, R. D., \& Hagins, W. A. Kinetics of the photocurrent of retinal rods. Biophysics, 1972, 12, 1073-1094.

Phillips, W. A., \& Singer, W. Function and interaction of on and off transients in vision. I: Psychophysics. Experimental Brain Research, 1974, 19, 493-506.

Pollack, I. Visual discrimination of "unseen" objects: Forcedchoice testing of Mayzner-Tresselt sequential blanking effects. Perception \& Psychophysics, 1972, 11, 121-128.

Pollack, I. Interaction effects in successive visual displays: An extension of the Eriksen-Collins paradigm. Perception \& Psychophysics, 1973, 13, 367-373.

RAINS, J. D. Reaction time to onset and cessation of a visual stimulus. Psychological Record, 1961, 11, 265-268.

Ross, J., \& HogBen, J. H. Short-term memory in stereopsis. Vision Research, 1974, 14, 1195-1201.

Russel., R. J. H. Temporal coding in iconic memory. Unpublished D.Phil. thesis, University of Oxford, 1977.

SAK ITT, B. Locus of short-term visual storage. Science, 1975, 190 , 1318-1319.

SA itт, B. Iconic memory. Psychological Review, 1976, 83, 257. 276. (a)

SAKITT, B. Psychophysical correlates of photoreceptor activity. Vision Research, 1976, 16, 129-140. (b)

SAKITT, B., \& Appelman, J. B. The effects of memory load and the contrast of the rod signal on partial report superiority in a Sperling task. Memory \& Cognition, 1978, 6, 562-567.

SakitT, B., \& Long, G. M. Relative rod and cone contributions to iconic storage. Perception \& Psychophysics, 1978, 23, 527536.

Sakitt, B., \& Long, G. M. Spare the rod and spoil the icon. Journal of Experimental Psychology; Human Perception and Performance, 1979, 5, 19-30.

Scharf, B., \& Lefton, L. A. Backward and forward masking as a function of stimulus and task parameters. Journal of Experimental Psychology, 1970, 84, 331-338.

Servière, J., Miceli, D., \& Galifret, Y. A psychophysical study of the visual perception of "instantaneous" and "durable." Vision Research, 1977, 17, 57-63. (a)

Skrvit̀re, J., Miceli, D., \& Galifret, Y. Electrophysiological correlates of the visual perception of "instantaneous" and 
"durable." Vision Research, 1977, 17, 65-69. (b)

Shallice, T. The detection of change and the perceptual moment hypothesis. British Journal of Statistical Psychology, 1964, 17, 113-135

Shallice, T., \& Warrington, E. K. The possible role of selective attention in acquired dyslexia. Neuropsychologia, $1977,15,31-41$

Singer, W. Temporal aspects of subcortical contrast processing. Neurosciences Research Programme Bulletin, 1977, 15, 358-369.

Singer, W., \& Phillips, W. A. Function and interaction of on and off transients in vision. II. Neurophysiology. Experimental Brain Research, 1974, 19, 507-521.

SPERLING, G. The information available in brief visual presentations. Psychological Monographs, 1960, 74, 1-29.

SPerling, G. Successive approximations to a model for shortterm memory. Acta Psychologia, 1967, 27, 285.292.

Stabell, U., \& Stabell, B. Absence of rod activity from peripheral vision. Vision Research, 1976, 16, 1433-1437.

STANLEY, G. Visual information processing and specific reading disability (dyslexia). In R. H. Day \& G. Stanley (Eds.), Studies in perception. Perth: University of Western Australia Press, 1978.

Stanley, G., \& Hall, R. Short-term visual information processing in dyslexics. Child Development, 1973, 44, 341-344.

Stanley, G., \& Molloy, M. Retinal painting and visual information storage. Acta Psychologica, 1975, 39, 283-288.

Steinberg, R. H. The rod after-effect in S-potentials from the cat retina. Vision Research, 1969, 9, 1345-1355.

Strouv, J. M. The moment function hypothesis. M.A. thesis, Stanford University, 1948.

Stroud, J. M. The fine structure of psychological time. In H. Quastler (Ed.), Information theory in psychology. Glencoe, Ill: Free Press, 1955.

Townsend, V. M. Loss of spatial and identity information following a tachistoscopic exposure. Journal of Experimental Psychology, 1973, 98, 113-118.

Treisman, A. M. Contextual cues in selective listening. Quarterly Journal of Experimental Psychology, 1960, 12, 242-248.

Trfisman, A. M., Russell, R., \& Green, J. Brief visual storage of shape and movement. In P. M. A. Rabbitt \& S. Dornic (Eds.), Attention and performance V. London: Academic Press, 1975.

Tulving, E. Episodic and semantic memory. In E. Tulving and W. Donaldson (Eds.), Organization of memory. New York: Academic Press, 1972.

Turvey, M. T. On peripheral and central processes in vision. Psychological Review, 1973, 80, 1-52.

Turvey, M. T. Visual processing and short-term memory. In W. K. Estes (Ed.), Handbook of learning and cognitive processes (Vol. 5). Hillsdale: Erlbaum, 1978.

Turvey, M. T., \& Kravetz, S. Retrieval from iconic and memory with shape as the selection criterion. Perception \& Psychophysics, 1970, 8, 171-172.
VAN Der Heijden, A. H. C. Short-term visual information forgetting. Unpublished PhD thesis, University of Leiden, The Netherlands, 1978.

Vassilev, A., \& Mitov, D. Perception time and spatial frequency. Vision Research, 1976, 16, 89-92.

von Wright, J. M. Selection in immediate visual memory. Quarterly Journal of Experimental Psychology, 1968, 20, 62-68.

W ADE, N. J. Some perceptual effects generated by rotating gratings. Perception, 1974, 3, 169-184.

Wässle, H., Levick, W. R., \& Cleland, B. G. The distribution of the alpha type of ganglion cells in the cat's retina. Journal of Comparative Neurology, 1975, 159, 419-437.

Westheimer, G. The Maxwellian view. Vision Research, 1966, 6, 669-682.

Whitten, D. N., \& Brown, K. T. The time courses of late receptor potentials from monkey cones and rods. Vision Research, 1973, 13, 107-135. (a)

Whitten, D. N., \& Brown, K. T. Photopic suppression of monkey's rod receptor potential, apparently by a cone-initiated lateral inhibition. Vision Research, 1973, 13, 1629-1658. (b)

Whitten, D. N., \& Brown, K. T. Slowed decay of the monkey's cone receptor potential by intense stimuli, and protection from this effect by light adaptation. Vision Research, 1973, 13, 1659-1667. (c)

\section{NOTES}

1. Mollon and Polden (1978) argued that both the rise and fall times of the tachistoscope bulbs used by Banks and Barber are different for different wavelengths of the bulb output when exposure duration is short. Hence, they claimed that scotopic discrimination of letter from background might be possible when brief exposures are used. This seems unlikely; and, in any case, it turns out that these letters were not visible to a rod monochromat even with brief exposures (Banks, personal communication).

2. To a lesser extent, this is also a difficulty for some of the other techniques discussed here (though not all of them).

3. The luminance values in the published paper are incorrect; the actual luminances used were $3,140,31.4, .314$, and $.198 \mathrm{~mL}$.

4. This objection was suggested by $C$. Blakemore.

5. This was pointed out to me by A. H. C. van der Heijden and $\mathrm{N}$. J. Wade.

6. Compare with the earlier quotation from Allport (1970).

7. His italics.

8. This finding needs to be reconciled with the observation that the visible persistences generated by stroboscopic illumination of a colored moving object are themselves all colored: this point was discussed earlier.

(Invited paper received September 27, 1979; reviewed, and accepted December 6, 1979.) 